\author{
Universidade de Brasília \\ Instituto de Ciências Exatas \\ Departamento de Matemática
}

\title{
Problemas Elípticos com Peso e Crescimento Crítico
}

\author{
por \\ Bruno Nunes de Souza \\ Orientador: Prof. Dr. Marcelo Fernandes Furtado
}

Brasília

2014 
Universidade de Brasília

Instituto de Ciências Exatas

Departamento de Matemática

\section{Problemas Elípticos com Peso e Crescimento Crítico}

Tese apresentada ao Departamento de Matemática da Universidade de Brasília como parte dos requisitos necessários para obtenção do grau de DOUTOR EM MATEMÁTICA

por

Bruno Nunes de Souza

Orientador: Prof. Marcelo Fernandes Furtado

13 de Novembro de 2014 
Universidade de Brasília

Instituto de Ciências Exatas

Departamento de Matemática

\section{Problemas Elípticos com Peso e Crescimento Crítico}

por

Tese apresentada ao Departamento de Matemática da Universidade de Brasília como parte dos requisitos necessários para obtenção do grau de

\section{DOUTOR EM MATEMÁTICA}

13 de Novembro de 2014

Comissão Examinadora:

Prof. Dr. Marcelo Fernandes Furtado (MAT/UnB)

Prof. Dr. Jiazheng Zhou (MAT/UnB)

Prof. Dr. Ricardo Ruviaro (MAT/UnB)

Prof. Dr. Everaldo Souto de Medeiros (MAT/UFPB)

Prof. Dr. Lucas Catão de Freitas Ferreira (MAT/Unicamp) 
"Como não ter Deus?! Com Deus existindo, tudo dá esperança: sempre um milagre é possível, o mundo se resolve. Mas, se não tem Deus, há-de a gente perdidos no vai-vem, e a vida é burra. É o aberto perigo das grandes e pequenas horas, não se podendo facilitar - é todos contra os acasos. Tendo Deus, é menos grave se descuidar um pouquinho, pois, no fim dá certo. Mas, se não tem Deus, então, a gente não tem licença de coisa nenhuma! Porque existe dôr. E a vida do homem está presa encantoada - erra rumo, dá em aleijões como esses, dos meninos sem pernas e braços. Dôr não dói até em criancinhas e bichos, e nos dôidos - não dói sem precisar de se ter razão nem conhecimento? E as pessoas não nascem sempre? Ah, medo tenho não é de ver morte, mas de ver nascimento. Medo mistério. O senhor não vê? O que não é Deus, é estado do demônio. Deus existe mesmo quando não há. Mas o demônio não precisa existir para haver - a gente sabendo que ele não existe, aí é que ele toma conta de tudo. O inferno é um sem-fim que nem não se pode ver. Mas a gente quer Céu é porque quer um fim: mas um fim com depois dele a gente tudo vendo. Se eu estou falando às flautas, o senhor me corte. Meu modo é este. Nasci para não ter homem igual em meus gostos. O que eu invejo é sua instrução do senhor..."

Grande Sertão: Veredas, de Guimarães Rosa. 
Agradeço à Deus, por tudo.

Agradeço ao meu orientador Marcelo. Por ser exemplo para mim de pequisador, professor, servidor público e pessoa. Por se esforçar tanto para que o mundo seja melhor. E pela paciência, nesses quase seis anos de convívio.

Aos professores da banca: Lucas, Everaldo, Ricardo e Zhou, pelos conselhos importantíssimos para o presente presente trabalho tanto quanto pelas dicas de futuros estudos.

Agradeço aos meus pais, José e Evangelina. À minha mãe, fonte inesgotável de amor. Ao meu pai, por ter me dado os melhores exemplos de vida.

Às minhas irmãs, Bel e Polli. Pelo amor e o carinho incondicional nos poucos momentos que estivemos juntos. E por elas terem me dado os melhores sobrinhos do mundo, Gabriel, Daniel, Kalebe, Arthur e Analu, com os quais eu pude passar momentos muito felizes.

À minha namorada Gislene, que me proporcionou nos últimos meses muito carinho e momentos de descontração. E por me fazer tão bem, por estar tão bem ao meu lado, bicho grilo que sou.

Agradeço a todos os amigos que fiz em quase sete anos de Brasília. Sem vocês, a vida não faria sentido: Marcelo Bezerra, Tarcísio, Weslley, Bruno César, Hudson, Laura, Simone, Nilton, João Paulo, Dani, Robson, Reinaldo, Thiago, Henrique, Elson, Eduardo, Andréia, Kali, Ilana, José Carlos, Mônica. E mais uma vez, agradeço especialmente à minha grande amiga Mariana que, além da bela amizade proporcionada, em 2007 me incentivou a vir estudar em Brasilia.

À Thaynara, a mais sincera amizade que tive nos últimos anos. Thay, estar com você é estar mais perto de quem eu realmente sou. 
Agradeço muito aos meus amigos de Uberaba. Especialmente Flávio, Deisemara, Rafael, Daniel e Laurinho. E por último ao Júlio, por nossas conversas sobre todos os assuntos relevantes da vida. Aprendi muito com todos vocês.

Este trabalho é uma pequena gota nas águas do conhecimento. No entanto, representa um mar de crescimento na vida do autor. Representa uma síntese de aprendizados, de passagens, de momentos de reflexão, de conversas em mesas de boteco, de entendimentos e desentendimentos. É a compilação das boas contribuições das pessoas que por mim passaram. Mas acima de tudo, é a resposta sobre o que a educação pode representar na vida de um homem. Ou, de uma criança. 
Neste trabalho, estudaremos a existência de soluções para equações do tipo

$$
-\operatorname{div}(p(x) \nabla u)=b(x)|u|^{q-2} u+c(x)|u|^{r-2} u, \quad x \in \Omega
$$

em que os pesos $p, b$ e $c$ satisfazem hipóteses que nos permitirão tratar o problema variacionalmente. Consideramos o problema acima para $1<q<2^{*}$ e tratamos, especialmente, variações para quando $r=2^{*}$ é o expoente crítico de Sobolev. A principal ferramenta utilizada será o Teorema do Passo da Montanha e suas versões.

Palavras-Chaves: Teorema do Passo da Montanha; pesos; expoente crítico de Sobolev. 
In this work, we will study the existence of solutions for the equation

$$
-\operatorname{div}(p(x) \nabla u)=b(x)|u|^{q-2} u+c(x)|u|^{r-2} u, \quad x \in \Omega,
$$

with the weights $p, b$ and $c$ verifying some hypothesis which produce a variational structure for the prolem. We considered the equation for $1<q<2^{*}$ and deal specially with the critical case $r=2^{*}$. We use the Mountain Pass Theorem as well as some of your variants.

Keywords: Mountain Pass Theorem; weights; Sobolev critical exponent. 
Introdução

1 Perturbações lineares e superlineares $\quad 10$

1.1 Solução nodal para $q=2 \ldots \ldots \ldots \ldots \ldots$. . . . . . . . . . . 12

1.2 Solução positiva para $2<q<2^{*} \ldots \ldots \ldots \ldots \ldots$. . . . . . . 28

1.3 Estimativas do Lema $1.2 \ldots \ldots$. . . . . . . . . . . . . . . . . 33

2 Perturbações do tipo côncavo-convexo 44

2.1 Lemas auxiliares . . . . . . . . . . . . . . . . . . . . . . 45

2.2 Prova do Teorema $2.1 \ldots \ldots \ldots \ldots$. . . . . . . . . . . . . . . . 50

2.3 Prova do Teorema $2.2 \ldots \ldots \ldots \ldots \ldots$. . . . . . . . . . . . . 53

3 Sistema com perturbação superlinear $\quad 62$

3.1 Resultado de não existência . . . . . . . . . . . . . . . . . . . . . . . . 64

3.2 Algumas relações importantes . . . . . . . . . . . . . . . . . . . . . 71

3.3 Existência de solução . . . . . . . . . . . . . . . . . . . . . 76

$\begin{array}{ll}\text { Referências Bibliográficas } & 85\end{array}$ 
Neste trabalho estudaremos a existência de soluções não negativas e nodais para problemas elípticos em domínios limitados suaves. Os dois primeiros capítulos serão dedicados ao estudo de equações do tipo

$$
\left\{\begin{aligned}
-\operatorname{div}(p(x) \nabla u) & =b(x)|u|^{q-2} u+c(x)|u|^{r-2} u, & & x \in \Omega \\
u & =0 & & x \in \partial \Omega
\end{aligned}\right.
$$

em que os potenciais $p, b$ e $c$ satisfazem hipóteses que nos permitirão tratar o problema (1) variacionalmente. Investigaremos o problema acima para variações das funções $b$ e $c$ e dos expoentes $q$ e $r$. Vale ressaltar que daremos maior atenção ao caso em que $r=2^{*}=2 N /(N-2)$, isto é, aquele em que temos crescimento crítico. Em todos os casos, a função $p$ satisfaz:

$\left(p_{1}\right) p \in H^{1}(\Omega) \cap C(\Omega) ;$

$\left(p_{2}\right)$ existe um ponto $a \in \Omega$ tal que

$$
p(a)=p_{0}:=\min \{p(x): x \in \bar{\Omega}\}>0
$$

$\left(p_{3}\right)$ existe $k>0, \beta_{k}>0$ e $\theta$ tais que, numa vizinhança de $a$, a função $p$ é da forma

$$
p(x)=p_{0}+\beta_{k}|x-a|^{k}+\theta(x)|x-a|^{k}
$$

$\operatorname{com} \lim _{x \rightarrow a} \theta(x)=0$. 
Um exemplo de uma função que satisfaz as condições acima é dado por

$$
p(x)=1+|x-a|^{2}+|x-a|^{s+2}, \quad x \in \Omega,
$$

em que $a \in \Omega$ e $s>0$. Nesse caso, ao definirmos $\theta(x)=|x-a|^{s}$, esta satisfaz a condição $\left(p_{3}\right)$.

A grande motivação para o estudo equação (1) se deve ao conceituado artigo de BrézisNirenberg [9], publicado em 1983. Os autores estudaram a existência de soluções positivas para o problema

$$
\left\{\begin{aligned}
-\Delta u & =\lambda|u|^{q-2} u+|u|^{2^{*}-2} u, & & x \in \Omega \\
u & =0, & & x \in \partial \Omega
\end{aligned}\right.
$$

e mostraram que a existência de solução está relacionada com a maneira que o parâmetro $\lambda$ se relaciona com o espectro do operador $-\Delta$. Com a devida precisão, denotando por $\lambda_{1}$ o primeiro auto-valor de $\left(-\Delta, H_{0}^{1}(\Omega)\right)$, eles obtiveram os seguintes resultados no caso em que $q=2$ :

- se $N \geq 3$ e $\lambda \geq \lambda_{1}$, então $(B N)$ não tem solução positiva;

- se $N \geq 4$ e $0<\lambda<\lambda_{1}$, então $(B N)$ tem solução positiva;

- se $N=3$, então existe $\lambda^{*}>0$ tal que $(B N)$ tem solução positiva sempre que $\lambda^{*}<\lambda<\lambda_{1}$

e se $2<q<2^{*}$ também provaram que:

- se $N=3$ e $4<q<6$, então $(B N)$ tem solução positiva para todo $\lambda>0$;

- se $N=3$ e $2<q \leq 4$, então $(B N)$ tem solução positiva para todo $\lambda>0$ suficientemente grande;

- se $N \geq 4$, então $(B N)$ tem solução positiva para todo $\lambda>0$.

Devido à falta de compacidade da imersão $H_{0}^{1}(\Omega) \hookrightarrow L^{2^{*}}(\Omega)$, o funcional energia $I$ associado ao problema $(B N)$ não satisfaz a condição de Palais-Smale em todos os níveis. Para contornar esse fato, provaram que $I$ satisfaz a condição $(P S)_{d}$ para todo

$$
d<c^{*}:=\frac{1}{N} S^{N / 2}
$$


em que $S$ é a melhor constante da imersão $H_{0}^{1}(\Omega) \hookrightarrow L^{2^{*}}(\Omega)$, dada por

$$
S=\inf \left\{\int|\nabla u|^{2}: u \in H_{0}^{1}(\Omega), \int|u|^{2^{*}}=1\right\}
$$

Após verificar que $I$ satisfaz a geometria do Passo da Montanha, eles mostraram que o nível minimax deste teorema está abaixo de $c^{*}$. Este último fato é o passo crucial da prova e é feito utilizando-se a função

$$
u_{\varepsilon}(x):=\varepsilon^{\frac{2-N}{2}} \psi(x) U(x / \varepsilon)
$$

em que $\varepsilon>0, \psi$ é uma função corte e a função $U$ é aquela introduzida por Aubin [3] e Talenti [32] como sendo

$$
U(x):=\frac{[N(N-2)]^{\frac{N-2}{4}}}{\left[1+|x|^{2}\right]^{\frac{N-2}{2}}}, \quad x \in \mathbb{R}^{N} .
$$

Em todos os capítulos desta tese, utilizaremos com frequência uma variação da função $u_{\varepsilon}$ citada acima.

Em 1985, Capozzi, Fortunato e Palmieri [10] provaram que o problema $(B N)$ tem solução não-trivial mesmo quando $\lambda \geq \lambda_{1}$. Naturalmente, esta solução tem que mudar de sinal, tendo em vista o resultado de não existência de solução positiva provado por Brezis e Nirenberg. Outros resultado foram estudados nesse mesmo contextos. Dentre eles citamos $[12,11,7,4,23,20,30,21,27,34]$.

Em 2007, Hadiji e Yazidi [24] investigaram existência de soluções positivas para o seguinte problema

$$
\left\{\begin{aligned}
-\operatorname{div}(p(x) \nabla u) & =\lambda|u|^{q-2} u+|u|^{2^{*}-2} u, & & x \in \Omega, \\
u & =0, & & x \in \partial \Omega,
\end{aligned}\right.
$$

em que $q=2$ e a função $p$ satisfaz $\left(p_{1}\right)-\left(p_{3}\right)$. Nesse caso, denotando por

$$
\lambda_{1, p}=\inf \left\{\int_{\Omega} p(x)|\nabla u|^{2} d x: u \in H_{0}^{1}(\Omega), \int_{\Omega} u^{2} d x=1\right\},
$$

o primeiro auto-valor de $\left(-\operatorname{div}(p(x) \nabla \cdot), H_{0}^{1}(\Omega)\right)$, a existência de solução positiva é garantida desde que sejam satisfeitas as seguintes hipóteses:

i) $N \geq 4, k>2$ e $0<\lambda<\lambda_{1, p}$;

ii) $N \geq 4, k=2$ e $\widetilde{\gamma}(N)<\lambda<\lambda_{1, p}$; 
iii) $N=3, k \geq 2$ e $\gamma(k)<\lambda<\lambda_{1, p}$;

iv) $N \geq 3,0<k<2$ e $\lambda^{*}<\lambda<\lambda_{1, p}$,

em que $\tilde{\gamma}, \gamma(k)$ e $\lambda^{*}$ são constantes positivas.

O trabalho acima tem grande importância em todo nosso estudo. Inspirados pela maneira que os resultados de Brézis-Niremberg foram complementados por Capozzi, Fortunato e Palmieri, estudamos o problema $(P)$ para $\lambda \geq \lambda_{1, p}$.

No restante da introdução, apresentaremos os resultados obtidos no nosso trabalho, fazendo um paralelo com outros resultados conhecidos na literatura.

\section{Pertubações lineares e superlineares}

No nosso primeiro resultado, estudamos a existência de solução para o problema $(P)$ quando $\lambda \geq \lambda_{1, p}$. Mais especificamente provamos o

Teorema 0.1. Se $q=2$ e p satisfaz $\left(p_{1}\right)-\left(p_{3}\right)$, então o problema $(P)$ tem pelos menos uma solução que muda de sinal em cada um dos casos abaixo:

1. $N \geq 4, k>2$ e $\lambda \geq \lambda_{1, p}$;

2. $N \geq 5, k=2$ e $\lambda \geq \max \left\{\lambda_{1, p}, \vartheta(N)\right\}$, em que

$$
\vartheta(N)= \begin{cases}4 \beta_{2}, & \text { se } N=4, \\ \vartheta_{0} \beta_{2}, & \text { se } N \geq 5,\end{cases}
$$

em que $\beta_{2}>0$ é dado em $\left(p_{3}\right)$ e

$$
\vartheta_{0}=(N-2)^{2}\left(\int_{\mathbb{R}^{N}} \frac{|y|^{4}}{\left(1+|y|^{2}\right)^{N}} d y\right)\left(\int_{\mathbb{R}^{N}} \frac{1}{\left(1+|y|^{2}\right)^{N-2}} d y\right)^{-1}
$$

3. $N=4, k=2, \lambda \geq \max \left\{\lambda_{1, p}, \vartheta(N)\right\}$ e existe $l_{0}>0$ tal que

$$
\int_{B\left(a, l_{0}\right)} \frac{\theta(x)}{|x-a|^{4}} d x<\infty .
$$

A demonstração desse teorema segue as linhas de [18], onde um problema em $\mathbb{R}^{N}$ foi estudado. Incialmente, provamos um resultado de compacidade local. Assim como foi 
observado em [9], nosso funcional energia $I$ não satisfaz $(P S)_{d}$ em todos os níveis. Porém, vale o seguinte resultado: se $\left(u_{n}\right) \subset H_{0}^{1}(\Omega)$ satisfaz

$$
\lim _{n \rightarrow+\infty} I\left(u_{n}\right)=c<c^{*}:={\frac{\left(p_{0} S\right)^{N / 2}}{N}}^{\text {e }} \lim _{n \rightarrow \infty} I^{\prime}\left(u_{n}\right)=0
$$

então $\left(u_{n}\right)$ possui uma subsequência convergente. A prova segue então de uma aplicação do Teorema de Linking. Um ponto importante para o nosso resultado é a prova de que o nível minimax se situa onde há compacidade. Neste ponto utilizamos algumas estimativas provadas em [24] (veja Seção 1.3).

No segundo resultado do Capítulo 1, consideramos o caso em que a pertubação é superlinear:

Teorema 0.2. Se $2<q<2^{*}$ e p satisfaz $\left(p_{1}\right)-\left(p_{3}\right)$ com $k>2$, então o problema $(P)$ tem pelos menos uma solução positiva para todo $\lambda>0$.

Na prova aplicamos o Teorema do Passo da Montanha. Novamente, a parte delicada é a correta estimativa do nível minimax associado ao funcional energia. Algumas adaptações dos argumentos apresentados em [18] são utilizados.

Os resultados provados no Capítulo 1 complementam os de [24] em dois sentidos: primeiro, porque consideramos a existência de solução que troca de sinal para $q=2$; segundo, porque tratamos o caso em que a perturbação do termo crítico e superlinear (e subcrítico). Estes resultados complementam, ainda, os resultados de Furtado, Myiagaki e Silva [18], que estudaram um problema análogo. De fato, em [18] os autores consideram um problema no $\mathbb{R}^{N}$ com o operador $\mathcal{F}(u)=\operatorname{div}(K(x) \nabla u)$, em que $K(x)=\exp \left(|x|^{\alpha} / 4\right)$.

\section{Pertubações do tipo côncavo-convexo}

No Capítulo 2 estudamos a existência de solução não negativa para o problema

$$
\left\{\begin{aligned}
-\operatorname{div}(p(x) \nabla u) & =b(x)|u|^{q-2} u+c(x)|u|^{r-2} u, & & x \in \Omega, \\
u & =0, & & x \in \partial \Omega
\end{aligned}\right.
$$

em que $\Omega \subset \mathbb{R}^{N}$ é um domínio suave e limitado, $N \geq 4$ e $1<q<2<r \leq 2^{*}=2 N /(N-2)$. Para $s>1$, denotamos por $s^{\prime}$ o expoente conjugado de $s$, a saber $s^{\prime}=s /(s-1)$. A função $p$ satisfaz $\left(p_{1}\right)-\left(p_{3}\right)$ e os potenciais $b$ e $c$ satisfazem 
$\left(b_{1}\right) b \in L^{\sigma_{q}}(\Omega)$ para algum

$$
\left(\frac{r}{q}\right)^{\prime}<\sigma_{q} \leq\left(\frac{2}{q}\right)^{\prime}
$$

$\left(c_{1}\right) c \in L^{\infty}(\Omega), \operatorname{com} c \not \equiv 0$.

Neste caso, nossa principal referência é o trabalho de autoria de Ambrosetti, Brezis e Cerami [2], de 1994, no qual estudaram o caso em que $b(x) \equiv \mu$ é um parâmetro e $p(x) \equiv c(x) \equiv 1$, provando que existe uma constante $\Lambda \in(0,+\infty)$ tal que o problema ( $P C$ ) tem duas soluções se $\mu<\Lambda$, pelo menos uma solução se $\mu=\Lambda$ e nenhuma solução se $\mu>\Lambda$. Posteriormente, de Figueiredo, Gossez e Ubilla [14], generalizaram esses resultados permitindo que os potenciais $b$ e $c$ fossem não constantes e mudassem de sinal. Outros resultados foram estudados para este problema, a saber [22, 33, 16, 15].

Nosso primeiro resultado trata do caso subcrítico:

Teorema 0.3. Suponha que $1<q<2<r<2^{*}$, a função $p$ satisfaz $\left(p_{1}\right)-\left(p_{3}\right)$ com $k>2$, as funções $b$ e c satisfazem $\left(b_{1}\right)$ e $\left(c_{1}\right)$. Se $|b|_{\sigma_{q}}$ é suficientemente pequeno, então o problema $(P C)$ tem pelos menos duas soluções não negativas $u_{0}$ e $u_{1}$, satisfazendo $u_{0}, u_{1} \neq 0$.

A prova deste teorema segue os mesmo argumentos de [2]. Usando a pequenez de $|b|_{\sigma_{q}}$ obtemos uma solução $u_{0} \neq 0$, via minimização. Em seguida, usamos o Teorema do Passo da Montanha centrado no mínimo local $u_{0}$ para a obtenção da segunda solução. Na parte técnica adaptamos algumas contas encontradas em [19], onde um problema relacionado (em domínio ilimitado) foi estudado. Neste caso, não conseguimos provar positividade das soluções pois, como os potenciais $b$ e $c$ podem mudar de sinal, não é possível utilizar princípio de máximo.

O segundo teorema do Capítulo 2 trata do caso crítico. A prova se torna um pouco mais complicada e precisamos da seguinte condição técnica:

$\left(b c_{1}\right)$ existe $\delta>0$ tal que $B_{\delta}(a) \subset\left(\Omega_{b}^{+} \cap \Omega_{c}^{+}\right)$e

$$
|c|_{\infty}-c(x) \leq M|x-a|^{\gamma}
$$

q.t.p. em $B_{\delta}(a)$, em que $\Omega_{b}^{+}:=\{x \in \Omega: b(x)>0\}, \Omega_{c}^{+}:=\{x \in \Omega: c(x)>0\}$, $M>0$ e $\gamma>(N-2) / 2$.

Provamos o seguinte 
Teorema 0.4. Suponha que $r=2^{*}$, p satisfaz $\left(p_{1}\right)-\left(p_{3}\right)$ com $N<(2 k+2)$, as funções $b$ e c satisfazem $\left(b_{1}\right),\left(c_{1}\right)$ e $\left(b c_{1}\right)$. Se $|b|_{\sigma_{q}}$ é suficientemente pequeno, então o problema $(P C)$ tem pelos menos duas soluções não negativas $u_{0}$ e $u_{1}$, satisfazendo $u_{0}, u_{1} \neq 0$.

A maior dificuldade na prova deste teorema é a obtenção da segunda solução. De fato, como temos crescimento crítico, o funcional energia associado não satisfaz Palais-Smale em todos os níveis. Após obter um resultado de compacidade local, provamos que o nível minimax está corretamente localizado. Neste ponto, além da condição técnica $\left(b c_{1}\right)$, é fundamental a restrição $N<(2 k+2)$. Vale notar que uma restrição relacionada já aparece no Teorema 1.2 do artigo de Furtado, Ruviaro e Silva [19]. De fato, os autores consideram um problema no $\mathbb{R}^{N}$ com o operador $\mathcal{F}(u)=\operatorname{div}(K(x) \nabla u)$, em que $K(x)=\exp \left(|x|^{\alpha} / 4\right)$ e a restrição imposta é $\alpha>(N-2) / 2$.

Nossos resultados do Capítulo 2 generalizam os resultados de [14] pois consideramos o caso em que $p(x)$ não é constante. Além disso usamos algumas estimativas de [24] para completar o resultado.

\section{Sistema com perturbação superlinear}

No Capítulo 3 estudamos existência de solução com componentes positivas para o sistema

$$
\left\{\begin{aligned}
-\operatorname{div}(p(x) \nabla u) & =b u+c v+\frac{\alpha}{2^{*}} u|u|^{\alpha-2}|v|^{\beta}, & & x \in \Omega, \\
-\operatorname{div}(q(x) \nabla v) & =c u+d v+\frac{\beta}{2^{*}}|u|^{\alpha} v|v|^{\beta-2}, & & x \in \Omega, \\
u, v & =0, & & x \in \partial \Omega,
\end{aligned}\right.
$$

em que $b, c, d \in \mathbb{R}$ e $\alpha, \beta>1$ são tais que $\alpha+\beta=2^{*}$. A função $p$ é como antes e a função $q$ satisfaz:

$\left(q_{1}\right) q \in H^{1}(\Omega) \cap C(\Omega) ;$

$\left(q_{2}\right) q(a)=q_{0}:=\min \{q(x): x \in \bar{\Omega}\}>0 ;$

$\left(q_{3}\right)$ existe $j>0, \gamma_{j}>0$ e $\theta_{q}$ tais que, numa vizinhança de $a$, a função $q$ é da forma

$$
q(x)=q_{0}+\gamma_{j}|x-a|^{j}+\theta_{q}(x)|x-a|^{j},
$$

$\operatorname{com} \lim _{x \rightarrow a} \theta_{q}(x)=0$. 
O caso em que $p(x) \equiv q(x) \equiv 1$ foi estudado por Alves, de Morais Filho e Souto [1] em 2003. Os resultados estão relacionados com os auto-valores $\mu_{1} \leq \mu_{2}$ da matriz real

$$
A=\left(\begin{array}{ll}
b & c \\
c & d
\end{array}\right) .
$$

Os autores provaram que, se $N \geq 4, b \geq 0$ e $0<\mu_{1} \leq \mu_{2}<\lambda_{1}$, então o sistema $(P Q)$ tem solução. Se $\Omega$ é um domínio estrelado com respeito à origem e $\mu_{2} \leq 0$, utilizando um tipo de identidade de Pohozaev, também provaram que $(P Q)$ só admite solução trivial. No mesmo contexto, outros trabalhos foram abordados no últimos anos, a saber $[25,26,17,5]$.

Primeiramente provamos um resultado de não existência de solução para o problema $(P Q)$, qual seja:

Teorema 0.5. Suponha que $\Omega$ é um domínio estrelado com respeito ao ponto a $\in \Omega$ e que $p, q \in C^{1}(\bar{\Omega})$ satisfazem

$$
\nabla p(x) \cdot(x-a) \geq 0 \quad \text { e } \nabla q(x) \cdot(x-a) \geq 0
$$

para todo $x \in \Omega$. Se $\mu_{2} \leq 0$, então o problema $(P Q)$ não possui solução positiva.

Para provar o resultado acima, utilizamos uma versão da identidade de Pohozaev para sistemas. Observe que, além de supormos que $\Omega$ é um domínio estrelado com respeito ao ponto $a$, acrescentamos as seguintes hipóteses técnicas sobre os pesos $p$ e $q$ :

$$
\nabla p(x) \cdot(x-a) \geq 0 \quad \text { e } \nabla q(x) \cdot(x-a) \geq 0
$$

Essas hipóteses surgem de maneira natural, tendo em vista que, diferentemente da identidade clássica de Pohozaev, não valem algumas relações do cálculo elementar, em função dos pesos $p(x)$ e $q(x)$.

Finalizamos o capítulo provando um resultado de existência de soluções positivas para o sistema $(P Q)$. Definindo $\lambda_{1, q}$ de maneira análoga à maneira com que foi definido $\lambda_{1, p} \mathrm{e}$ a quantidade

$$
\vartheta(N, k, j):= \begin{cases}0, & \text { se } \min \{k, j\}>2, \\ \vartheta_{1} \beta_{2}, & \text { se } k=2, j>2, \\ \vartheta_{1} \gamma_{2}, & \text { se } k>2, j=2, \\ \vartheta_{1} \max \left\{\beta_{2}, \gamma_{2}\right\}, & \text { se } k=2, j=2,\end{cases}
$$


em que

$$
\vartheta_{1}:=\left\{\begin{array}{l}
4, \text { se } N=4, \\
(N-2)^{2}\left(\int_{\mathbb{R}^{N}} \frac{|y|^{4}}{\left(1+|y|^{2}\right)^{N}} d y\right)\left(\int_{\mathbb{R}^{N}} \frac{1}{\left(1+|y|^{2}\right)^{N-2}} d y\right)^{-1}, \text { se } N \geq 5,
\end{array}\right.
$$

podemos então enunciar nosso último resultado:

Teorema 0.6. Se as funções p e q satisfazem $\left(p_{1}\right)-\left(p_{3}\right)$ e $\left(q_{1}\right)-\left(q_{3}\right)$, a matriz A é tal que $c>0$ e

$$
\vartheta(N, k, j)<\mu_{1} \leq \mu_{2}<\min \left\{\lambda_{1, p}, \lambda_{1, q}\right\}
$$

então o problema $(P Q)$ tem pelo menos uma solução com componetes positivas.

O teorema acima é provado usando-se o Teorema do Passo da Montanha. A parte mais delicada é a correta localização do nível minimax do funcional energia. Novamente, a criticalidade do sistema faz com que este não satisfaça Palais-Smale em todos níveis. Para efetivar os cálculos necessários usamos algumas ideias do artigo [1].

Ressaltamos que, após a finalização dos resultados deste último capítulo, tomamos conhecimento do artigo [6], em que os autores obtêm resultados semelhantes aos nossos. Uma vez que nossos resultados foram obtidos paralelamente e de forma independente, optamos por apresentá-los nesta tese. 


\section{CAPÍTULO 1}

\section{Perturbações lineares e superlineares}

Neste capítulo, investigamos a existência de solução para o problema

$$
\left\{\begin{aligned}
-\operatorname{div}(p(x) \nabla u) & =\lambda|u|^{q-2} u+|u|^{2^{*}-2} u, & & x \in \Omega, \\
u & =0, & & x \in \partial \Omega,
\end{aligned}\right.
$$

em que $\Omega \subset \mathbb{R}^{N}$ é um domínio suave e limitado, $N \geq 4,2^{*}=2 N /(N-2)$ é o expoente crítico de Sobolev e $2 \leq q<2^{*}$. A função $p$ satisfaz as seguintes condições:

$\left(p_{1}\right) p \in H^{1}(\Omega) \cap C(\Omega) ;$

$\left(p_{2}\right)$ existe um ponto $a \in \Omega$ tal que

$$
p(a)=p_{0}:=\min \{p(x): x \in \bar{\Omega}\}>0
$$

$\left(p_{3}\right)$ existe $k>0, \beta_{k}>0$ e $\theta$ tais que, numa vizinhança de $a$, a função $p$ é da forma

$$
p(x)=p_{0}+\beta_{k}|x-a|^{k}+\theta(x)|x-a|^{k},
$$

com $\lim _{x \rightarrow a} \theta(x)=0$.

O parâmetro $\lambda>0$ vai variar de acordo com o valor de $q$ e estará relacionado, no caso $q=2$, com o número

$$
\lambda_{1, p}=\inf \left\{\int p(x)|\nabla u|^{2}: u \in H_{0}^{1}(\Omega), \int u^{2}=1\right\}
$$


O nosso primeiro resultado trata do caso em que $q=2$ :

Teorema 1.1. Se $q=2$ e p satisfaz $\left(p_{1}\right)-\left(p_{3}\right)$, então o problema $(P)$ tem pelos menos uma solução que muda de sinal em cada um dos casos abaixo:

1. $N \geq 4, k>2$ e $\lambda \geq \lambda_{1, p}$;

2. $N \geq 5, k=2$ e $\lambda \geq \max \left\{\lambda_{1, p}, \vartheta(N)\right\}$, em que

$$
\vartheta(N)= \begin{cases}4 \beta_{2}, & \text { se } N=4, \\ \vartheta_{0} \beta_{2}, & \text { se } N \geq 5\end{cases}
$$

em que $\beta_{2}>0$ é dado em $\left(p_{3}\right)$ e

$$
\vartheta_{0}=(N-2)^{2}\left(\int_{\mathbb{R}^{N}} \frac{|y|^{4}}{\left(1+|y|^{2}\right)^{N}} d y\right)\left(\int_{\mathbb{R}^{N}} \frac{1}{\left(1+|y|^{2}\right)^{N-2}} d y\right)^{-1} .
$$

3. $N=4, k=2, \lambda \geq \max \left\{\lambda_{1, p}, \vartheta(N)\right\}$ e existe $l_{0}>0$ tal que

$$
\int_{B\left(a, l_{0}\right)} \frac{\theta(x)}{|x-a|^{4}} d x<\infty .
$$

No segundo resultado, consideramos o caso em que a pertubação é superlinear:

Teorema 1.2. Se $2<q<2^{*}$ e p satisfaz $\left(p_{1}\right)-\left(p_{3}\right)$ com $k>2$, então o problema $(P)$ tem pelos menos uma solução positiva para todo $\lambda>0$.

Em todo o capítulo vamos considerar o espaço $H_{0}^{1}(\Omega)$ com a sua norma usual

$$
\|u\|^{2}=\int|\nabla u|^{2}, \quad \forall u \in H_{0}^{1}(\Omega)
$$

Vamos denotar ainda por $\|\cdot\|_{p}$ a norma

$$
\|u\|_{p}^{2}=\int p(x)|\nabla u|^{2}, \quad u \in H_{0}^{1}(\Omega)
$$

induzida pelo produto interno

$$
\langle u, v\rangle_{p}=\int p(x)(\nabla u \cdot \nabla v), \quad \forall u, v \in H_{0}^{1}(\Omega)
$$


Observamos que a norma $\|\cdot\|_{p}$ definida em (1.1) é equivalente à norma usual $\|\cdot\|$ de $H_{0}^{1}(\Omega)$, pois

$$
p_{0}\|u\|^{2}=p_{0} \int|\nabla u|^{2} \leq \int p(x)|\nabla u|^{2}=\|u\|_{p}^{2} \leq|p|_{\infty}\|u\|^{2} .
$$

Nas duas seções seguintes provamos os Teoremas 1.1 e 1.2, respectivamente. O capítulo conta ainda com uma seção final onde fazemos algumas estimativas utilizadas no decorrer das provas.

\subsection{Solução nodal para $q=2$}

Nesta seção vamos considerar $q=2$, de forma que as soluções fracas do problema $(P)$ são precisamente pontos críticos do funcional $I \in C^{1}\left(H_{0}^{1}(\Omega), \mathbb{R}\right)$ definido por

$$
I(u):=\frac{1}{2} \int p(x)|\nabla u|^{2}-\frac{\lambda}{2} \int u^{2}-\frac{1}{2^{*}} \int|u|^{2^{*}}
$$

Para encontrar tais pontos críticos, vamos utilizar o seguinte teorema abstrato, cuja demonstração encontra-se em [29]:

Teorema 1.3. Seja $X$ um espaço de Banach com $X=Y \oplus Z$ e $\operatorname{dim} Y<\infty$. Considere um funcional $J \in C^{1}(X, \mathbb{R})$ satisfazendo:

(i) existem $\rho, \sigma>0$ tais que $\left.J\right|_{\partial B_{\rho}(0) \cap Z} \geq \sigma$;

(ii) existem e $\in \partial B_{1}(0) \cap Z$ e $R>\rho$ tais que

$$
\left.J\right|_{\partial Q} \leq 0
$$

em que $Q=\left(\overline{B_{R}(0)} \cap Y\right) \oplus\{$ te $: 0<t<R\}$.

Seja

$$
c=\inf _{\gamma \in \Gamma} \max _{u \in Q} J(\gamma(u))
$$

em que

$$
\Gamma=\{\gamma \in C(\bar{Q}, X): \gamma \equiv I d \quad \text { em } \partial Q\} .
$$

Então $c \geq \sigma$ e existe $\left(u_{n}\right) \subset X$ tal que $J\left(u_{n}\right) \rightarrow$ c e $J^{\prime}\left(u_{n}\right) \rightarrow 0$.

Devido à falta de compacidade da imersão $H_{0}^{1}(\Omega) \hookrightarrow L^{2^{*}}(\Omega)$, o funcional $I$ não satisfaz 
a condição de Palais-Smale em todos os níveis. Denotando

$$
S=\inf \left\{\int|\nabla u|^{2}: u \in H_{0}^{1}(\Omega), \int|u|^{2^{*}}=1\right\}
$$

o resultado abaixo fornece uma condição de compacidade local.

Lema 1.1. Se $\left(u_{n}\right) \subset H_{0}^{1}(\Omega)$ é tal que

$$
\lim _{n \rightarrow+\infty} I\left(u_{n}\right)=c<c^{*}:={\frac{\left(p_{0} S\right)^{N / 2}}{N}}^{N} \quad \text { lim } \lim _{n \rightarrow \infty} I^{\prime}\left(u_{n}\right)=0
$$

então $\left(u_{n}\right)$ possui uma subsequência convergente.

Demonstração: Tomemos $\beta \in\left(\frac{1}{2^{*}}, \frac{1}{2}\right)$. Para $n$ suficientemente grande temos que $\left\|I^{\prime}\left(u_{n}\right)\right\| \leq 1 / \beta$. Assim

$$
\left|I^{\prime}\left(u_{n}\right) u_{n}\right| \leq\left\|I^{\prime}\left(u_{n}\right)\right\|\left\|u_{n}\right\| \leq(1 / \beta)\left\|u_{n}\right\|
$$

e portanto $\left\|u_{n}\right\| \geq-\beta I^{\prime}\left(u_{n}\right) u_{n}$. Como $I\left(u_{n}\right)=c+o(1)$, da desigualdade acima temos que

$$
\begin{aligned}
o(1)+c+\left\|u_{n}\right\| & \geq I\left(u_{n}\right)-\beta I^{\prime}\left(u_{n}\right) u_{n} \\
& =\frac{1}{2} \int p(x)\left|\nabla u_{n}\right|^{2}-\frac{\lambda}{2} \int\left|u_{n}\right|^{2}-\frac{1}{2^{*}} \int\left|u_{n}\right|^{2^{*}} \\
& -\beta \int p(x)\left|\nabla u_{n}\right|^{2}+\beta \lambda \int\left|u_{n}\right|^{2}+\beta \int\left|u_{n}\right|^{2^{*}},
\end{aligned}
$$

e portanto

$$
o(1)+c+\left\|u_{n}\right\| \geq\left(\frac{1}{2}-\beta\right)\left\|u_{n}\right\|_{p}^{2}-\lambda\left(\frac{1}{2}-\beta\right)\left|u_{n}\right|_{2}^{2}+\left(\beta-\frac{1}{2^{*}}\right)\left|u_{n}\right|_{2^{*}}^{2^{*}}
$$

Uma consequência da desigualdade de Young é que, para quaisquer $a, b \geq 0$ e $\varepsilon>0$, existe $C_{\varepsilon}$ tal que

$$
a b \leq \varepsilon a^{s}+C_{\varepsilon} b^{s^{\prime}}
$$

em que $1 / s+1 / s^{\prime}=1$. Devido à imersão $L^{2^{*}}(\Omega) \hookrightarrow L^{2}(\Omega)$ e à desigualdade acima, existem constantes $c_{1}, c_{2}>0$ tais que

$$
\left|u_{n}\right|_{2} \leq c_{1}\left|u_{n}\right|_{2^{*}} \leq \varepsilon\left|u_{n}\right|_{2^{*}}^{2^{*} / 2}+c_{2}
$$


Segue de (1.2), da igualdade acima e da condição $\left(p_{2}\right)$ que

$$
\begin{aligned}
o(1)+c+\left\|u_{n}\right\| & \geq p_{0} \beta_{0}\left\|u_{n}\right\|^{2}-\lambda \beta_{0}\left(\varepsilon\left|u_{n}\right|_{2^{*}}^{2^{*} / 2}+c_{2}\right)^{2}+\beta_{1}\left|u_{n}\right|_{2^{*}}^{2^{*}} \\
& \geq p_{0} \beta_{0}\left\|u_{n}\right\|^{2}-\lambda \beta_{0} c_{3} \varepsilon^{2}\left|u_{n}\right|_{2^{*}}^{2^{*}}-c_{4}+\beta_{1}\left|u_{n}\right|_{2^{*}}^{2^{*}}
\end{aligned}
$$

em que $\beta_{0}=(1 / 2-\beta), \beta_{1}=\left(\beta-1 / 2^{*}\right), c_{3}$ e $c_{4}$ são constantes positivas. Escolhendo $0<\varepsilon<\sqrt{\beta_{1}}\left(\lambda \beta_{0} c_{3}\right)^{-1 / 2}$, teremos

$$
o(1)+c+\left\|u_{n}\right\| \geq p_{0} \beta_{0}\left\|u_{n}\right\|^{2}-c_{4}
$$

donde concluímos que $\left(u_{n}\right)$ é limitada em $H_{0}^{1}(\Omega)$.

Resta-nos mostrar que $\left(u_{n}\right)$ possui uma subsequência convergente. Como $\left(u_{n}\right)$ é limitada, a menos de subsequência, existe $u \in H_{0}^{1}(\Omega)$ tal que

$$
u_{n} \rightarrow u \text { fracamente em } H_{0}^{1}(\Omega) \quad \text { e } \quad u_{n}(x) \rightarrow u(x) \text { q.t.p. em } \Omega \text {. }
$$

Usando a compacidade da imersão $H_{0}^{1}(\Omega) \hookrightarrow L^{2^{*}-1}(\Omega)$, a menos de subsequência, existe $h_{s} \in L^{s}(\Omega)$ tal que

$$
\begin{aligned}
u_{n} & \rightarrow u \text { em } L^{2}(\Omega), \\
u_{n} & \rightarrow u \text { em } L^{s}(\Omega), \\
\left|u_{n}(x)\right| & \leq h_{s}(x), \text { q.t.p. em } \Omega
\end{aligned}
$$

em que $s=2^{*}-1$. Assim, para toda $\phi \in C_{0}^{\infty}(\Omega)$,

$$
\begin{aligned}
u_{n}^{s}(x) \phi(x) & \rightarrow u^{s}(x) \phi(x) \text { q.t.p. em } \Omega, \\
\left|u_{n}\right|^{s}|\phi| & \leq|\phi|_{\infty} h_{s}^{s} \in L^{1}(\Omega) .
\end{aligned}
$$

Pelo Teorema da Convergência Dominada,

$$
\int\left|u_{n}\right|^{2^{*}-2} u_{n} \phi \longrightarrow \int|u|^{2^{*}-2} u \phi
$$

e, por densidade,

$$
\int\left|u_{n}\right|^{2^{*}-2} u_{n} v \longrightarrow \int|u|^{2^{*}-2} u v, \quad \forall v \in H_{0}^{1}(\Omega)
$$


Como $u_{n} \rightarrow u$ em $H_{0}^{1}(\Omega), u_{n} \rightarrow u$ em $L^{2}(\Omega)$ e $p \in C(\bar{\Omega})$

$$
\left\langle u_{n}, v\right\rangle_{p}-\lambda \int u_{n} v=\langle u, v\rangle_{p}-\lambda \int u v+o(1), \forall v \in H_{0}^{1}(\Omega) .
$$

Lembrando que $I^{\prime}\left(u_{n}\right) \rightarrow 0$, obtemos

$$
\begin{aligned}
o(1) & =I^{\prime}\left(u_{n}\right) v \\
& =\left\langle u_{n}, v\right\rangle_{p}-\lambda \int u_{n} v-\int\left|u_{n}\right|^{2^{*}-2} u_{n} v \\
& =\langle u, v\rangle_{p}-\lambda \int u v-\int|u|^{2^{*}-2} u v+o(1),
\end{aligned}
$$

para toda $v \in H_{0}^{1}(\Omega)$, o que mostra que $u$ é solução fraca do problema $(P)$.

Um resultado devido a Brézis-Lieb [8] nos diz que

$$
\left|u_{n}\right|_{q}^{q}=|u|_{q}^{q}+\left|u_{n}-u\right|_{q}^{q}+o(1)
$$

para $1 \leq q \leq 2^{*}$. Assim, se denotarmos $v_{n}=: u_{n}-u$, temos

$$
\begin{aligned}
o(1) & =I^{\prime}\left(u_{n}\right) u_{n} \\
& =\left\|u_{n}\right\|_{p}^{2}-\lambda\left|u_{n}\right|_{2}^{2}-\left|u_{n}\right|_{2^{*}}^{2^{*}} \\
& =\|u\|_{p}^{2}+\left\|u_{n}-u\right\|_{p}^{2}-\lambda|u|_{2}^{2}-\lambda\left|u_{n}-u\right|_{2}^{2}-|u|_{2^{*}}^{2^{*}}-\left|u_{n}-u\right|_{2^{*}}^{2^{*}}+o(1) \\
& =I^{\prime}(u) u+\left\|v_{n}\right\|_{p}^{2}-\lambda\left|v_{n}\right|_{2}^{2}-\left|v_{n}\right|_{2^{*}}^{2^{*}}+o(1) \\
& =\left\|v_{n}\right\|_{p}^{2}-\left|v_{n}\right|_{2^{*}}^{2^{*}}+o(1),
\end{aligned}
$$

em que usamos o fato de que $I^{\prime}(u) u=0$. Portanto, para algum $l \geq 0$, vale

$$
\lim _{n \rightarrow+\infty}\left\|v_{n}\right\|_{p}^{2}=\lim _{n \rightarrow+\infty}\left|v_{n}\right|_{2^{*}}^{2^{*}}=l
$$

Mas segue das definições de $S$ e $p(x)$ que

$$
\int p(x)\left|\nabla v_{n}\right|^{2} \geq p_{0} \int\left|\nabla v_{n}\right|^{2} \geq p_{0} S\left|v_{n}\right|_{2^{*}}^{2} .
$$

Passando ao limite a inequação acima e usando (1.4), obtemos

$$
l \geq p_{0} S l^{2 / 2^{*}}
$$


Suponhamos $l \neq 0$. Então $l \geq\left(p_{0} S\right)^{N / 2}>0$. Por (1.3) temos que

$$
\begin{aligned}
I\left(u_{n}\right) & =\frac{1}{2}\left\|u_{n}\right\|_{p}^{2}-\frac{\lambda}{2}\left|u_{n}\right|_{2}^{2}-\frac{1}{2^{*}}\left|u_{n}\right|_{2^{*}}^{2^{*}} \\
& =\frac{1}{2}\left\|v_{n}+u\right\|_{p}^{2}-\frac{\lambda}{2}|u|_{2}^{2}-\frac{1}{2^{*}}\left|v_{n}+u\right|_{2^{*}}^{2^{*}}+o(1) \\
& =\frac{1}{2}\|u\|_{p}^{2}-\frac{\lambda}{2}|u|_{2}^{2}-\frac{1}{2}|u|_{2^{*}}^{2^{*}}+\frac{1}{2}\left\|v_{n}\right\|_{p}^{2}-\frac{1}{2^{*}}\left|v_{n}\right|_{2^{*}}^{2^{*}}+o(1) \\
& =I(u)+\frac{1}{2}\left\|v_{n}\right\|_{p}^{2}-\frac{1}{2^{*}}\left|v_{n}\right|_{2^{*}}^{2^{*}}+o(1)
\end{aligned}
$$

Portanto, usando (1.4), a igualdade acima e o fato de que $I\left(u_{n}\right) \rightarrow c$, obtemos

$$
I(u)+\left(\frac{1}{2}-\frac{1}{2^{*}}\right) l=c
$$

Além disso, como $I^{\prime}(u) u=0$, temos que

$$
\begin{aligned}
I(u) & =I(u)-\frac{1}{2} I^{\prime}(u) u \\
& =\left(\frac{1}{2}-\frac{1}{2^{*}}\right)|u|_{2^{*}}^{2^{*}} \geq 0 .
\end{aligned}
$$

Logo, as igualdades acima implicam que

$$
c \geq\left(\frac{1}{2}-\frac{1}{2^{*}}\right) l=\frac{1}{N} l
$$

e portanto devemos ter

$$
c^{*}={\frac{\left(p_{0} S\right)}{N}}^{N / 2} \leq \frac{l}{N} \leq c<c^{*}
$$

o que gera uma contradição. Portanto $l=0$, e isto conclui a demonstração do lema, pois $\left\|v_{n}\right\|_{p}^{2}=o(1)$ e, portanto, $u_{n} \rightarrow u$ em $H_{0}^{1}(\Omega)$.

Nosso próximo passo é decompor o espaço $H_{0}^{1}(\Omega)$ de tal maneira que possa ser verificada a geometria do nosso teorema abstrato. Para isso, vamos considerar o problema linear

$\left(P_{\lambda}^{\operatorname{div}}\right) \quad\left\{\begin{aligned}-\operatorname{div}(p(x) \nabla u) & =\lambda u, & & x \in \Omega \\ u & =0, & & x \in \partial \Omega\end{aligned}\right.$ 
Nesse caso, vale o seguinte resultado, cuja a prova é uma consequência da Alternativa de Fredholm:

Teorema 1.4. Se $\Omega \subset \mathbb{R}^{N}$ é um aberto limitado, então o problema de auto-valor $\left(P_{\lambda}^{d i v}\right)$ possui uma sequência de auto-valores

$$
0<\lambda_{1, p}<\lambda_{2, p} \leq \lambda_{3, p} \leq \cdots \leq \lambda_{n, p} \leq \lambda_{n+1, p} \rightarrow \infty
$$

Além disso, as auto-funções associadas, que definiremos por $\varphi_{1, p}, \varphi_{2, p}, \ldots, \varphi_{n, p}, \cdots$, formam uma base ortogonal de $H_{0}^{1}(\Omega)$.

Assim, dado $n \in \mathbb{N}$ tal que $\lambda_{n, p} \leq \lambda<\lambda_{n+1, p}$, definimos

$$
Y:=\operatorname{span}\left\{\varphi_{1, p}, \varphi_{2, p}, \ldots, \varphi_{n, p}\right\}, \quad Z:=Y^{\perp}
$$

e a constante

$$
\delta=\inf _{\substack{u \in Z \\\|u\|=1}} \int\left(p(x)|\nabla u|^{2}-\lambda u^{2}\right) .
$$

Como consequência da Teoria Spectral dos Operadores Compactos seguem as seguintes desigualdades:

$$
\int p(x)|\nabla u|^{2} \leq \lambda_{n, p} \int u^{2}, \quad \forall u \in Y
$$

$\mathrm{e}$

$$
\int p(x)|\nabla u|^{2} \geq \lambda_{n+1, p} \int u^{2}, \quad \forall u \in Z \text {. }
$$

Assim, dado $u \in Z$, com $\|u\|=1$, da última desigualdade, do fato de $\lambda<\lambda_{n+1, p}$ e de $\left(p_{2}\right)$, obtemos

$$
\int\left(p(x)|\nabla u|^{2}-\lambda u^{2}\right) \geq\left(1-\frac{\lambda}{\lambda_{n+1, p}}\right) \int p(x)|\nabla u|^{2} \geq\left(1-\frac{\lambda}{\lambda_{n+1, p}}\right) p_{0}>0
$$

donde concluímos que $\delta>0$.

O próximo resultado será importante na prova do Teorema 1.1. Sua demonstração será feita mais adiante.

Proposição 1.1. Se $\lambda \in\left[\lambda_{n, p}, \lambda_{n+1, p}\right)$ satisfaz uma das condições (1)-(3) do Teorema 1.1, então existe $z \in Z \backslash\{0\}$ tal que

$$
\max _{u \in Y \oplus \mathbb{R} z} I(u)<c^{*}=\frac{1}{N}\left(p_{0} S\right)^{N / 2} .
$$


Assumindo que a proposição é verdadeira, podemos provar o Teorema 1.1 como se segue:

Demonstração do Teorema 1.1: Consideremos $Y, Z$ e $\delta$ definidos em (1.5) e (1.6). Se $u \in Z$, então

$$
\begin{aligned}
I(u) & =\frac{1}{2} \int p(x)|\nabla u|^{2}-\frac{\lambda}{2} \int u^{2}-\frac{1}{2^{*}} \int|u|^{2^{*}} \\
& \geq \frac{\delta}{2}\|u\|^{2}-\frac{1}{2^{*}}|u|_{2^{*}}^{2^{*}}
\end{aligned}
$$

Da definição de $S$ temos que $|u|_{2^{*}}^{2^{*}} \leq S^{-2^{*} / 2}\|u\|^{2^{*}}$. Assim, segue da expressão acima que, se $\|u\|=\rho$ e $u \in Z$, então

$$
\begin{aligned}
I(u) & \geq \frac{\delta}{2} \rho^{2}-S^{-2^{*} / 2} \frac{\rho^{2^{*}}}{2^{*}} \\
& =\rho^{2}\left(\frac{\delta}{2}-\frac{S^{-2^{*} / 2}}{2} \rho^{2^{*}-2}\right)>0, \quad \forall u \in \partial B_{\rho}(0) \cap Z
\end{aligned}
$$

desde que $\rho<\left(\delta S^{2^{*} / 2}\right)^{1 /\left(2^{*}-2\right)}$. Para essa escolha de $\rho$ temos

$$
b=\inf _{u \in \partial B_{\rho}(0) \cap Z} I(u)>0
$$

o que assegura a condição (i) do Teorema 1.3.

Tendo em vista a desigualdade (1.7), para todo $u \in Y$, temos que

$$
\begin{aligned}
I(u) & =\frac{1}{2} \int p(x)|\nabla u|^{2}-\frac{\lambda}{2} \int u^{2}-\frac{1}{2^{*}} \int|u|^{2^{*}} \\
& \leq \frac{\lambda_{n, p}}{2} \int u^{2}-\frac{\lambda}{2} \int u^{2}-\frac{1}{2^{*}} \int|u|^{2^{*}} \\
& \leq \frac{\left(\lambda_{n, p}-\lambda\right)}{2} \int u^{2}-\frac{1}{2^{*}} \int|u|^{2^{*}} \\
& \leq 0 .
\end{aligned}
$$

Como $Y \oplus \mathbb{R} z$ tem dimensão finita, todas as normas neste espaço são equivalentes. Logo, existem $c_{2}, c_{3}, c_{4}>0$ tais que, para todo $u \in Y \oplus \mathbb{R} z$,

$$
\begin{aligned}
I(u) & =\frac{1}{2}\|u\|_{p}^{2}-\frac{\lambda}{2}|u|_{2}^{2}-\frac{1}{2^{*}}|u|_{2^{*}}^{2^{*}} \\
& \leq \frac{1}{2} c_{2}\|u\|^{2}-\frac{\lambda}{2} c_{3}\|u\|^{2}-\frac{1}{2^{*}} c_{4}\|u\|^{2^{*}},
\end{aligned}
$$


donde cocluímos que

$$
I(u) \longrightarrow-\infty, \text { sempre que }\|u\| \rightarrow+\infty, \quad u \in Y \oplus \mathbb{R} z
$$

Assim, existe $R>\rho$ tal que $I(u) \leq 0$ sempre que $\|u\|=R$. Concluímos que a condição (ii) do Teorema 1.3 é satisfeita.

Pelo Teorema 1.3 e a Proposição 1.1, existe uma sequência $\left(u_{n}\right) \subset H_{0}^{1}(\Omega)$ tal que

$$
\lim _{n \rightarrow+\infty} I\left(u_{n}\right)=c<c^{*}={\frac{\left(p_{0} S\right)^{N / 2}}{N}}^{\text {e }} \lim _{n \rightarrow \infty} I^{\prime}\left(u_{n}\right)=0
$$

Pelo Lema 1.1 podemos afirmar que, a menos de subsequência, $\left(u_{n}\right)$ converge para uma solução $u$ de $(P)$.

Para mostrar que $u \not \equiv 0$ usamos o Teorema 1.3, que garante que $I(u)=c \geq \sigma>0$, donde concluímos que a solução $u$ é não trivial. Resta-nos mostrar que a solução muda de sinal. De fato, pois caso contrário, como $u \not \equiv 0$, pelo Princípio do Máximo, deveríamos ter $u>0$ ou $u<0$. Mas como o Teorema 1.1 de [24] afirma que $(P)$ não tem solução positiva no caso $\lambda \geq \lambda_{1, p}$, concluímos que a solução encontrada tem que mudar de sinal.

Caminharemos agora na direção de demonstrar a Proposição 1.1. A prova será dividida em dois casos e, em ambos, vamos considerar o quociente

$$
Q_{\lambda, p}(u):=\frac{\|u\|_{p}^{2}-\lambda|u|_{2}^{2}}{|u|_{2^{*}}^{2}}, \quad u \in H_{0}^{1}(\Omega) \backslash\{0\},
$$

bem como a seguinte função

$$
w_{\varepsilon}(x):=\varepsilon^{\frac{N-2}{4}} u_{\varepsilon}(x)=\frac{\psi(x) \varepsilon^{\frac{N-2}{4}}}{\left[\varepsilon+|x-a|^{2}\right]^{(N-2) / 2}},
$$

em que $\varepsilon>0, \psi \in C_{0}^{\infty}(\Omega), 0 \leq \psi \leq 1, \psi \equiv 1$ em $B(a, l), \psi \equiv 0$ em $\Omega \backslash \overline{B(a, 2 l)}$ e $l>0$ é tal que $\overline{B(a, 2 l)} \subset \Omega$.

A seguir, enunciamos a relação entre as duas funções acima citadas. As estimativas do próximo lema foram extraídas de [24] e serão provadas na Seção 1.3.

Lema 1.2. Temos que $Q_{\lambda, p}\left(w_{\varepsilon}\right)=Q_{\lambda, p}\left(u_{\varepsilon}\right)$. Além disso, valem as seguintes estimativas 
quando $\varepsilon \rightarrow 0^{+}$

$$
Q_{\lambda, p}\left(w_{\varepsilon}\right) \leq \begin{cases}p_{0} S-\lambda \frac{K_{3}}{K_{2}} \varepsilon+o(\varepsilon), & N \geq 5 \text { e } k>2 ; \\ p_{0} S-\left(\lambda-\frac{A_{2}}{K_{3}} \beta_{2}\right) \frac{K_{3}}{K_{2}} \varepsilon+o(\varepsilon), & N \geq 5 \text { e } k=2 ; \\ p_{0} S-\lambda \frac{\omega_{4}}{2 K_{2}} \varepsilon|\log \varepsilon|+o(\varepsilon|\log \varepsilon|), & N=4 \text { e } k>2 ; \\ p_{0} S-\left(\lambda-4 \beta_{2}\right) \frac{\omega_{4}}{2 K_{2}} \varepsilon|\log \varepsilon|+o(\varepsilon|\log \varepsilon|), & N=4 \text { e } k=2,\end{cases}
$$

em que $\omega_{4}$ é a área da esfera unitária de $\mathbb{R}^{4}$,

$$
K_{2}:=\frac{1}{S}(N-2)^{2} \int_{\mathbb{R}^{N}} \frac{|y|^{2}}{\left[1+|y|^{2}\right]} d y, \quad K_{3}:=\int_{\mathbb{R}^{N}} \frac{1}{\left[1+|y|^{2}\right]^{N-2}} d y
$$

$e$

$$
A_{2}:=(N-2)^{2} \int_{\mathbb{R}^{N}} \frac{|y|^{4}}{\left(1+|y|^{2}\right)^{N}} d y .
$$

De posse das estimativas acima, vamos provar a Proposição 1.1, em seus dois casos, na sequência do texto.

Caso 1: $\lambda_{n, p}<\lambda<\lambda_{n+1, p}$.

Nesse primeiro caso, definimos

$$
z_{\varepsilon}:=w_{\varepsilon}-\sum_{i=1}^{n}\left(\int w_{\varepsilon} \varphi_{i, p}\right) \varphi_{i, p}
$$

em que $\varphi_{i, p}$ são as autofunções do problema de autovalor $\left(P_{\lambda}^{\text {div }}\right)$.

Observemos que, fixado $u \in H_{0}^{1}(\Omega) \backslash\{0\}$,

$$
I(t u)=\frac{t^{2}}{2}\|u\|_{p}^{2}-\frac{\lambda t^{2}}{2}|u|_{2}^{2}-\frac{t^{2^{*}}}{2^{*}}|u|_{2^{*}}^{2^{*}}
$$

Se $\|u\|_{p}^{2}-\lambda|u|_{2}^{2} \leq 0$, temos que $I(t u) \leq 0$ para todo $t \geq 0$. Caso contrário, a função $t \mapsto I(t u)$, para $t>0$, possui um único ponto crítico $t_{0}>0$ dado por

$$
t_{0}:=\left(\frac{\|u\|_{p}^{2}-\lambda|u|_{2}^{2}}{|u|_{2^{*}}^{2^{*}}}\right)^{1 /\left(2^{*}-2\right)} .
$$

Além disso, como $2^{*}>2$, um cálculo simples mostra que $t_{0}$ é um ponto de máximo, de modo que o máximo da função é dado por

$$
I\left(t_{0} u\right)=\frac{1}{N}\left(\frac{\|u\|_{p}^{2}-\lambda|u|_{2}^{2}}{|u|_{2^{*}}^{2}}\right)^{N / 2} .
$$


Assim, para todo $u \in H_{0}^{1}(\Omega) \backslash\{0\}$, vale

$$
\max _{t \geq 0} I(t u) \leq \frac{1}{N}\left(\frac{\|u\|_{p}^{2}-\lambda|u|_{2}^{2}}{|u|_{2^{*}}^{2}}\right)^{N / 2} .
$$

Logo, como $Y \oplus \mathbb{R} w_{\varepsilon}=Y \oplus \mathbb{R} z_{\varepsilon}$, se definirmos

$$
\Sigma_{\varepsilon}:=\left\{u=y+t w_{\varepsilon} ; y \in Y, t \in \mathbb{R},|u|_{2^{*}}=1\right\}
$$

a Proposição 1.1 fica provada se conseguirmos verificar a seguinte desigualdade

$$
m_{\varepsilon}:=\max _{u \in \Sigma_{\varepsilon}}\left(\|u\|_{p}^{2}-\lambda|u|_{2}^{2}\right)<p_{0} S
$$

Lema 1.3. Quando $\varepsilon \rightarrow 0^{+}$, as seguintes igualdades se verificam:

$$
\left|w_{\varepsilon}\right|_{2^{*}-1}^{2^{*}-1}=O\left(\varepsilon^{\frac{N-2}{4}}\right), \quad\left|w_{\varepsilon}\right|_{1}=O\left(\varepsilon^{\frac{N-2}{4}}\right)
$$

e para todo $y \in Y$

$$
\max \left\{\left|\left\langle y, w_{\varepsilon}\right\rangle_{p}\right|,\left|\int y w_{\varepsilon}\right|\right\}=|y|_{2} O\left(\varepsilon^{\frac{N-2}{4}}\right) .
$$

Demonstração: Observemos primeiramente que, dado $\gamma \neq 0$, podemos usar o Teorema da Mudança de Variável para obter

$$
\int_{B_{2 l}(a)} \frac{1}{\left[\varepsilon+|x-a|^{2}\right]^{\gamma}} d x=\varepsilon^{-\gamma+\frac{N}{2}} \int_{B_{2 l / \sqrt{\varepsilon}}(a)} \frac{1}{\left[1+|x|^{2}\right]^{\gamma}} d x .
$$

Como na definicão de $w_{\varepsilon}$ a função $\psi$ se anula exteriormente à bola $B(a, 2 l)$, podemos usar (1.15) com $\gamma=(N+2) / 2$ para obter

$$
\begin{aligned}
\left|w_{\varepsilon}\right|_{2^{*}-1}^{2^{*}-1} & =\int\left(\frac{\psi(x) \varepsilon^{(N-2) / 4}}{\left[\varepsilon+|x-a|^{2}\right]^{(N-2) / 2}}\right)^{\frac{N+2}{N-2}} \\
& \leq \varepsilon^{(N-2) / 4} \int_{B_{2 l / \sqrt{\varepsilon}}(0)} \frac{1}{\left[1+|x|^{2}\right]^{(N+2) / 2}} d x \\
& =O\left(\varepsilon^{\frac{N-2}{4}}\right) .
\end{aligned}
$$


Além disso,

$$
\begin{aligned}
\left|w_{\varepsilon}\right|_{1} & =\int \frac{\psi(x) \varepsilon^{(N-2) / 4}}{\left[\varepsilon+|x-a|^{2}\right]^{(N-2) / 2}} \\
& \leq \varepsilon^{(N-2) / 4} \int_{B(a, 2 l)} \frac{1}{\left[\varepsilon+|x-a|^{2}\right]^{(N-2) / 2}} d x \\
& \leq \varepsilon^{(N-2) / 4} \int_{B(a, 2 l)} \frac{1}{|x-a|^{(N-2)}} d x \\
& =O\left(\varepsilon^{\frac{N-2}{4}}\right),
\end{aligned}
$$

visto que a última integral é finita.

Para provar (1.14), tomemos um elemento $y=\sum_{i=1}^{n} \beta_{i} \varphi_{i, p}$ arbitrário de $Y$. Podemos nos valer de (1.13) para obter

$$
\begin{aligned}
\left|\left\langle y, w_{\varepsilon}\right\rangle_{p}\right| & =\left|\left\langle\sum_{i=1}^{n} \beta_{i} \varphi_{i, p}, w_{\varepsilon}\right\rangle_{p}\right|=\left|\sum_{i=1}^{n} \lambda_{i, p} \beta_{i} \int \varphi_{i, p} w_{\varepsilon}\right| \\
& \leq c_{3}\left(\sum_{i=1}^{n}\left|\beta_{i}\right|\right) \int w_{\varepsilon} \leq\left(\sum_{i=1}^{n}\left|\beta_{i}\right|\right) O\left(\varepsilon^{\frac{N-2}{4}}\right)
\end{aligned}
$$

em que $c_{3}=\lambda_{n, p} \max \left\{\left|\varphi_{1, p}\right|_{\infty}, \ldots,\left|\varphi_{n, p}\right|_{\infty}\right\}$. Além disso, a equivalência de normas em $Y$ implica que $\sum_{i=1}^{n}\left|\beta_{i}\right| \leq c_{4}|y|_{2}$. Utilizando essa desigualdade juntamente com (1.16), obtemos

$$
\left|\left\langle y, w_{\varepsilon}\right\rangle_{p}\right| \leq|y|_{2} O\left(\varepsilon^{\frac{N-2}{4}}\right) .
$$

Analogamente

$$
\left|\int y w_{\varepsilon}\right| \leq|y|_{2} O\left(\varepsilon^{\frac{N-2}{4}}\right)
$$

o que finaliza a demonstração do lema.

Lema 1.4. Se $u=y+t w_{\varepsilon} \in \Sigma_{\varepsilon}$, então $t=O(1)$ quando $\varepsilon \rightarrow 0^{+}$.

Demonstração: Seja

$$
A(u)=|u|_{2^{*}}^{2^{*}}-|y|_{2^{*}}^{2^{*}}-\left|t w_{\varepsilon}\right|_{2^{*}}^{2^{*}} .
$$


Pelo Teorema do Valor Médio

$$
\begin{aligned}
A(u) & =\int\left(\left|y+t w_{\varepsilon}\right|^{2^{*}}-|y|^{2^{*}}-\left|t w_{\varepsilon}\right|^{2^{*}}\right) \\
& =2^{*} \iint_{0}^{1}\left(\left|t w_{\varepsilon}+s y\right|^{2^{*}-2}\left(t w_{\varepsilon}+s y\right)-|s y|^{2^{*}-2} s y\right) d s \\
& =2^{*}\left(2^{*}-1\right) \iint_{0}^{1}\left|s y+t w_{\varepsilon} \eta(x)\right|^{2^{*}-2} t w_{\varepsilon} y d s,
\end{aligned}
$$

em que $0 \leq \eta(x) \leq 1$ é uma função mensurável. Como $\operatorname{dim} Y<\infty$, neste espaço todas as normas são equivalentes. Além disso, se $m, n$ e $\mu$ são constantes positivas, vale a desigualdade $(m+n)^{\mu} \leq C_{\mu}\left(m^{\mu}+n^{\mu}\right)$, para algum $C_{\mu}>0$. Assim, por (1.13)

$$
\begin{aligned}
|A(u)| & =\left|2^{*}\left(2^{*}-1\right) \iint_{0}^{1}\right| s y+\left.t w_{\varepsilon} \eta(x)\right|^{2^{*}-2} t w_{\varepsilon} y d s \mid \\
& \leq c_{1} 2^{*}\left(2^{*}-1\right)\left(\iint_{0}^{1}|s|^{2^{*}-2}|y|^{2^{*}-1}\left|t w_{\varepsilon}\right| d s+\iint_{0}^{1}\left|t w_{\varepsilon} \eta\right|^{2^{*}-2} t w_{\varepsilon}|y| d s\right) \\
& \leq c_{2}\left\{|y|_{\infty}^{2^{*}-1}|t|\left|w_{\varepsilon}\right|_{1}+|y|_{\infty}|t|^{2^{*}-1}\left|w_{\varepsilon}\right|_{2^{*}-1}^{2^{*}-1}\right\} \\
& \leq c_{3}\left\{|y|_{2^{*}}^{2^{*}-1}|t| O\left(\varepsilon^{\frac{N-2}{4}}\right)+|y|_{2^{*}}|t|^{2^{*}-1} O\left(\varepsilon^{\frac{N-2}{4}}\right)\right\} .
\end{aligned}
$$

Dado agora $\xi>0$, segue da desigualdade de Young com expoentes $r=2^{*} /\left(2^{*}-1\right)$ e $r^{\prime}=2^{*}$ que

$$
|y|_{2^{*}}^{2^{*}-1}|t| O\left(\varepsilon^{\frac{N-2}{4}}\right) \leq \xi|y|_{2^{*}}^{2^{*}}+c_{4}|t|^{2^{*}} O\left(\varepsilon^{\frac{N-2}{4}}\right)^{\frac{2 N}{N-2}} .
$$

Analogamente, existe uma constante $c_{5}$ tal que

$$
|y|_{2^{*}}|t|^{2^{*}-1} O\left(\varepsilon^{\frac{N-2}{4}}\right) \leq \xi|y|_{2^{*}}^{2^{*}}+c_{5}|t|^{2^{*}} O\left(\varepsilon^{\frac{N-2}{4}}\right)^{\frac{2 N}{N+2}} .
$$

Escolhendo $\xi<1 /\left(4 c_{3}\right)$, podemos usar (1.17) e as duas últimas desigualdades para garantir que

$$
|A(u)| \leq \frac{1}{2}|y|_{2^{*}}^{2^{*}}+|t|^{2^{*}}\left\{O\left(\varepsilon^{\frac{N}{2}}\right)+O\left(\varepsilon^{\frac{N(N-2)}{2(N+2)}}\right)\right\} .
$$

Portanto, usando a estimativa

$$
\left|w_{\varepsilon}\right|_{2^{*}}^{2^{*}}=\int\left|w_{\varepsilon}\right|^{2^{*}}=[N(N-2)]^{-2^{*}} S^{N / 2}+O\left(\varepsilon^{\frac{N}{2}}\right)
$$

obtida no artigo de Brezis-Nirenberg (cf. [9, pg 444]), a definição de $A(u)$ e (1.18), segue 
que

$$
\begin{aligned}
1 & =|u|_{2^{*}}^{2^{*}} \\
& \geq\left|t w_{\varepsilon}\right|_{2^{*}}^{2^{*}}+\frac{1}{2}|y|_{2^{*}}^{2^{*}}+|t|^{2^{*}}\left\{O\left(\varepsilon^{\frac{N}{2}}\right)+O\left(\varepsilon^{\frac{N(N-2)}{2(N+2)}}\right)\right\} \\
& \geq|t|^{2^{*}}\left\{[N(N-2)]^{-2^{*}} S^{N / 2}+O\left(\varepsilon^{\frac{N}{2}}\right)+O\left(\varepsilon^{\frac{N(N-2)}{2(N+2)}}\right)\right\} .
\end{aligned}
$$

Dessa maneira, se $\varepsilon \rightarrow 0^{+}$, devemos ter $t=O(1)$, o que finaliza a prova do lema.

Demonstração da Proposição 1.1 (Caso $\lambda_{n, p}<\lambda<\lambda_{n+1, p}$ ) Seja $u=y+t w_{\varepsilon} \in \Sigma_{\varepsilon}$, em que o conjunto $\Sigma_{\varepsilon}$ é aquele definido em (1.12). Usando a estimativa (1.14) do Lema 1.3 e o fato de que $t=O(1)$ quando $\varepsilon \rightarrow 0^{+}$(Lema 1.4) obtemos

$$
\begin{aligned}
\|u\|_{p}^{2} & =\|y\|_{p}^{2}+2 t\left\langle y, w_{\varepsilon}\right\rangle_{p}+\left\|t w_{\varepsilon}\right\|_{p}^{2} \\
& \leq\|y\|_{p}^{2}+|y|_{2} O\left(\varepsilon^{\frac{N-2}{4}}\right)+\left\|t w_{\varepsilon}\right\|_{p}^{2} .
\end{aligned}
$$

Argumentando como acima no cálculo de $\left|y+t w_{\varepsilon}\right|_{2}^{2}$ e usando a desigualdade (1.7), obtemos

$$
\|u\|_{p}^{2}-\lambda|u|_{2}^{2} \leq\left(\lambda_{n, p}-\lambda\right)|y|_{2}^{2}+|y|_{2} O\left(\varepsilon^{\frac{N-2}{4}}\right)+Q_{\lambda, p}\left(t w_{\varepsilon}\right)\left|t w_{\varepsilon}\right|_{2^{*}}^{2}
$$

em que $Q_{\lambda, p}$ foi definido em (1.10). Para $m<0$, temos que $m r^{2}+b r \leq-b^{2} / 4 m$, para todo $r \in \mathbb{R}$. Lembrando que $\left(\lambda_{n, p}-\lambda\right)<0$ e usando essa desigualdade, obtemos então

$$
\|u\|_{p}^{2}-\lambda|u|_{2}^{2} \leq \frac{1}{4\left(\lambda-\lambda_{n, p}\right)} O\left(\varepsilon^{\frac{N-2}{2}}\right)+Q_{\lambda, p}\left(t w_{\varepsilon}\right)\left|t w_{\varepsilon}\right|_{2^{*}}^{2}
$$

Por outro lado, usando o Teorema do Valor Médio e a equivalência das normas no espaço $Y$, existe uma função mensurável $\xi$, tomando valores no intervalo $[0,1]$, tal que

$$
\begin{aligned}
1 & =\int\left|y+t w_{\varepsilon}\right|^{2^{*}} \\
& =\int\left(\left|t w_{\varepsilon}\right|^{2^{*}}+2^{*}\left|t w_{\varepsilon}+\xi(x) y\right|^{2^{*}-2}\left(t w_{\varepsilon}+\xi(x) y\right) y\right) \\
& \geq\left|t w_{\varepsilon}\right|_{2^{*}}^{2^{*}}+2^{*} \int\left|t w_{\varepsilon}\right|^{2^{*}-1}|y| \\
& \geq\left|t w_{\varepsilon}\right|_{2^{*}}^{2^{*}}-|y|_{2} O\left(\varepsilon^{\frac{N-2}{4}}\right)
\end{aligned}
$$

donde segue que

$$
\left|t w_{\varepsilon}\right|_{2^{*}}^{2^{*}} \leq 1+|y|_{2} O\left(\varepsilon^{\frac{N-2}{4}}\right) .
$$


A conclusão é consequência, então, da estimativa acima, de (1.19) e do Lema 1.2. Suponhamos que $N \geq 5$ e $k>2$ e usando as estimativas citadas na frase anterior, temos que

$$
\|u\|_{p}^{2}-\lambda|u|_{2}^{2} \leq p_{0} S+\varepsilon\left(d+\frac{1}{4\left(\lambda-\lambda_{n, p}\right)} O\left(\varepsilon^{\frac{N-2}{4}}\right)+o(1)\right),
$$

em que $d=\lambda K_{3} / K_{2}<0$. Assim, para $\varepsilon>0$ suficientemente pequeno, temos que

$$
\|u\|_{p}^{2}-\lambda|u|_{2}^{2} \leq \gamma<p_{0} S, \quad \forall u \in \Sigma_{\varepsilon}
$$

Os demais casos são tratados de maneira análoga.

Caso 2: $\lambda=\lambda_{n, p}$.

Definimos

$$
\widetilde{w}_{\varepsilon}:=w_{\varepsilon}-\left\langle w_{\varepsilon}, \varphi_{n, p}\right\rangle_{p} \varphi_{n, p},
$$

em que $w_{\varepsilon}$ foi definido em (1.11). Mostraremos, de maneira análoga ao primeiro caso, que

$$
\widetilde{m}_{\varepsilon}=\max _{u \in \widetilde{\Sigma}_{\varepsilon}}\left(\|u\|_{p}^{2}-\lambda_{n, p}|u|_{2}^{2}\right)<p_{0} S
$$

em que

$$
\widetilde{\Sigma}_{\varepsilon}=\left\{u=y+t \widetilde{w}_{\varepsilon} ; y \in Y, t \in \mathbb{R},|u|_{2^{*}}=1\right\} \text {. }
$$

Lema 1.5. Quando $\varepsilon \rightarrow 0^{+}$, valem as seguintes estimativas

$$
\begin{gathered}
\left|\widetilde{w}_{\varepsilon}\right|_{2^{*}-1}^{2^{*}-1}=O\left(\varepsilon^{\frac{N-2}{4}}\right), \quad\left|\widetilde{w}_{\varepsilon}\right|_{1}=O\left(\varepsilon^{\frac{N-2}{4}}\right), \\
\max \left\{\left|\left\langle y, \widetilde{w}_{\varepsilon}\right\rangle_{p}\right|,\left|\int y \widetilde{w}_{\varepsilon}\right|\right\} \leq|y|_{2} O\left(\varepsilon^{\frac{N-2}{4}}\right),
\end{gathered}
$$

$e$

$$
Q_{\lambda, p}\left(\widetilde{w}_{\varepsilon}\right)=Q_{\lambda, p}\left(w_{\varepsilon}\right)+\frac{O\left(\varepsilon^{\frac{N-2}{2}}\right)}{\left|w_{\varepsilon}\right|_{2^{*}}^{2}+O\left(\varepsilon^{\frac{N-2}{2}}\right)}
$$


Demonstração: Para a primeira estimativa, temos que

$$
\begin{aligned}
\left|\widetilde{w}_{\varepsilon}\right|_{2^{*}-1}^{2^{*}-1} & =\int\left|\widetilde{w}_{\varepsilon}\right|^{2^{*}-1} \\
& =\int\left|w_{\varepsilon}-\left\langle w_{\varepsilon}, \varphi_{n, p}\right\rangle_{p} \varphi_{n, p}\right|^{2^{*}-1} \\
& \leq c_{1} \int\left|w_{\varepsilon}\right|^{2^{*}-1}+c_{2}\left|\left\langle w_{\varepsilon}, \varphi_{n, p}\right\rangle_{p}\right|^{2^{*}-1} \int\left|\varphi_{n, p}\right|^{2^{*}-1} \\
& =O\left(\varepsilon^{\frac{N-2}{4}}\right)+O\left(\varepsilon^{\frac{N-2}{4}}\right)^{\frac{N+2}{N-2}} \\
& =O\left(\varepsilon^{\frac{N-2}{4}}\right),
\end{aligned}
$$

em que usamos as equações (1.13) e (1.14). Analogamente, podemos nos valer do Lema 1.3 para provar a segunda e terceira igualdades do lema. Além disso, sabemos que

$$
\begin{aligned}
\left\|\widetilde{w}_{\varepsilon}\right\|_{p}^{2} & =\left\|w_{\varepsilon}\right\|_{p}^{2}+\left\langle w_{\varepsilon}, \varphi_{n, p}\right\rangle_{p}^{2}\left\|\varphi_{n, p}\right\|_{p}^{2}-2\left\langle w_{\varepsilon}, \varphi_{n, p}\right\rangle_{p}^{2} \\
& \leq\left\|w_{\varepsilon}\right\|_{p}^{2}+O\left(\varepsilon^{\frac{N-2}{4}}\right)^{2} \\
& =\left\|w_{\varepsilon}\right\|_{p}^{2}+O\left(\varepsilon^{\frac{N-2}{2}}\right)
\end{aligned}
$$

e analogamente

$$
\left|\widetilde{w}_{\varepsilon}\right|_{2}^{2} \leq\left|w_{\varepsilon}\right|_{2}^{2}+O\left(\varepsilon^{\frac{N-2}{2}}\right) .
$$

Estabeleceremos agora uma relação entre $\left|\widetilde{w}_{\varepsilon}\right|_{2^{*}}^{2}$ e $\left|w_{\varepsilon}\right|_{2^{*}}^{2}$ :

$$
\begin{aligned}
\left|\widetilde{w}_{\varepsilon}\right|_{2^{*}}^{2^{*}}-\left|w_{\varepsilon}\right|_{2^{*}}^{2^{*}} & =\iint_{0}^{1} \frac{d}{d s}\left|w_{\varepsilon}-s\left\langle w_{\varepsilon}, \varphi_{n, p}\right\rangle_{p} \varphi_{n, p}\right|^{2^{*}} d s \\
& \leq c_{2}\left|\left\langle w_{\varepsilon}, \varphi_{n, p}\right\rangle_{p}\right| \int\left|w_{\varepsilon}\right|^{2^{*}-1}+c_{3}\left|\left\langle w_{\varepsilon}, \varphi_{n, p}\right\rangle_{p}\right|^{2^{*}} \int\left|\varphi_{n, p}\right|^{2^{*}} \\
& =O\left(\varepsilon^{\frac{N-2}{4}}\right) O\left(\varepsilon^{\frac{N-2}{4}}\right)+O\left(\varepsilon^{\frac{N-2}{4}}\right)^{2^{*}} \\
& =O\left(\varepsilon^{\frac{N-2}{2}}\right),
\end{aligned}
$$

em que utilizamos novamente (1.13) e (1.14). Para algum $\xi \in(0,1)$, temos

$$
\begin{aligned}
\left|\widetilde{w}_{\varepsilon}\right|_{2^{*}}^{2} & =\left(\left|\widetilde{w}_{\varepsilon}\right|_{2^{*}}\right)^{2 / 2^{*}} \\
& =\left(\left|w_{\varepsilon}\right|_{2^{*}}^{2^{*}}+O\left(\varepsilon^{\frac{N-2}{2}}\right)\right)^{2 / 2^{*}} \\
& =\left(\left|w_{\varepsilon}\right|_{2^{*}}^{2^{*}}\right)^{2 / 2^{*}}+\frac{2}{2^{*}}\left(\left|w_{\varepsilon}\right|_{2^{*}}^{2^{*}}+\xi O\left(\varepsilon^{\frac{N-2}{2}}\right)\right)^{\frac{2}{2^{*}-1}} O\left(\varepsilon^{\frac{N-2}{2}}\right) .
\end{aligned}
$$


Como $0<\lim _{\varepsilon \rightarrow 0^{+}}\left|w_{\varepsilon}\right|_{2^{*}}<\infty$, concluímos que

$$
\left|\widetilde{w}_{\varepsilon}\right|_{2^{*}}^{2}=\left|w_{\varepsilon}\right|_{2^{*}}^{2}+O\left(\varepsilon^{\frac{N-2}{2}}\right)
$$

A equação acima, (1.20) e (1.21) implicam que

$$
\begin{aligned}
Q_{\lambda, p}\left(\widetilde{w}_{\varepsilon}\right) & =\frac{\left\|\widetilde{w}_{\varepsilon}\right\|_{p}^{2}-\lambda\left|\widetilde{w}_{\varepsilon}\right|_{2}^{2}}{\left|\widetilde{w}_{\varepsilon}\right|_{2^{*}}^{2}} \\
& \leq \frac{\left\|w_{\varepsilon}\right\|_{p}^{2}-\lambda\left|w_{\varepsilon}\right|_{2}^{2}+O\left(\varepsilon^{\frac{N-2}{2}}\right)}{\left|w_{\varepsilon}\right|_{2^{*}}^{2}+O\left(\varepsilon^{\frac{N-2}{2}}\right)} \\
& =Q_{\lambda, p}\left(w_{\varepsilon}\right)+\frac{O\left(\varepsilon^{\frac{N-2}{2}}\right)}{\left|w_{\varepsilon}\right|_{2^{*}}^{2}+O\left(\varepsilon^{\frac{N-2}{2}}\right)},
\end{aligned}
$$

finalizando, pois, a prova.

Demonstração da Proposição 1.1 (Caso $\lambda=\lambda_{n, p}$ ) A prova segue as mesmas linhas do primeiro caso. Provaremos que

$$
\widetilde{m}:=\max _{u \in \widetilde{\Sigma}_{\varepsilon}}\left(\|u\|_{p}^{2}-\lambda_{n, p}|u|_{2}^{2}\right)<p_{0} S
$$

em que

$$
\widetilde{\Sigma}_{\varepsilon}:=\left\{u=y+t \widetilde{w}_{\varepsilon}: y \in Y, t \in \mathbb{R},|u|_{2^{*}}=1\right\}
$$

Ao considerarmos $u=y+t \widetilde{w}_{\varepsilon} \in \widetilde{\Sigma}_{\varepsilon}$, a função $y \in Y$ pode ser reescrita como

$$
y=\widetilde{y}+\left\langle y, \varphi_{n, p}\right\rangle_{p} \varphi_{n, p}
$$

Dessa maneira $\left\langle\varphi_{n, p}, \widetilde{w}_{\varepsilon}\right\rangle_{p}=\int \varphi_{n, p} \widetilde{w}_{\varepsilon}=0$ e $\left\|\varphi_{n, p}\right\|_{p}^{2}=\lambda_{n, p}\left|\varphi_{n, p}\right|_{2}^{2}$, tendo em vista a definição de $\varphi_{n, p}$. Assim

$$
\|u\|_{p}^{2}-\lambda_{n, p}|u|_{2}^{2}=\|\widetilde{y}\|_{p}^{2}-\lambda_{n, p}|\widetilde{y}|_{2}^{2}+2\left\langle\widetilde{y}, t \widetilde{w}_{\varepsilon}\right\rangle_{p}-2 \lambda_{n, p} \int t \widetilde{y} \widetilde{w}_{\varepsilon}+Q_{\lambda}\left(t \widetilde{w}_{\varepsilon}\right)\left|t \widetilde{w}_{\varepsilon}\right|_{2^{*}}^{2}
$$

Como vimos no Lema 1.5, podemos obter

$$
\|u\|_{p}^{2}-\lambda_{n, p}|u|_{2}^{2} \leq \frac{1}{4\left(\lambda_{n-1, p}-\lambda_{n, p}\right)} O\left(\varepsilon^{\frac{N-2}{2}}\right)+Q_{\lambda}\left(t \widetilde{w}_{\varepsilon}\right)\left|t \widetilde{w}_{\varepsilon}\right|_{2^{*}}^{2}
$$


Utilizando o fato de que $t=O(1)$ quando $\varepsilon \rightarrow 0^{+}$(Lema 1.4) e o mesmo argumento usado no primeiro caso, para $\varepsilon$ suficientemente pequeno, teremos

$$
\|u\|_{p}^{2}-\lambda|u|_{2}^{2}<p_{0} S, \quad \forall u \in \widetilde{\Sigma}_{\varepsilon}
$$

finalizando, então, a prova da proposição.

\subsection{Solução positiva para $2<q<2^{*}$}

Nesta seção, vamos provar o Teorema 1.2. O roteiro é parecido com o da seção anterior, porém, neste caso, vamos aplicar o Teorema do Passo da Montanha para o funcional

$$
I(u):=\frac{1}{2} \int p(x)|\nabla u|^{2}-\frac{\lambda}{q} \int\left(u^{+}\right)^{q}-\frac{1}{2^{*}} \int\left(u^{+}\right)^{2^{*}},
$$

em que $u^{+}(x)=\max \{u(x), 0\}$. Começaremos enunciando e demonstrando o seguinte lema que fornece a geometria do Teorema do Passo da Montanha.

Lema 1.6. Existem $\rho, \sigma>0$ tais que $\left.I\right|_{\partial B_{\rho}(0)} \geq \sigma$. Além disso, existe $e \in H_{0}^{1}(\Omega)$ tal que $\|e\| \geq \rho e I(e)<0$.

Demonstração: Usando as imersões de Sobolev, obtemos $c_{1}, c_{2}>0$ tais que

$$
\begin{aligned}
I(u) & =\frac{1}{2}\|u\|_{p}^{2}-\frac{\lambda}{q}\left|u^{+}\right|_{q}^{q}-\frac{1}{2^{*}}\left|u^{+}\right|_{2^{*}}^{2^{*}} \\
& \geq \frac{p_{0}}{2}\|u\|^{2}-\frac{\lambda}{q}\left|u^{+}\right|_{q}^{q}-\frac{1}{2^{*}}\left|u^{+}\right|_{2^{*}}^{2^{*}} \\
& \geq \frac{p_{0}}{2}\|u\|^{2}-c_{1}\|u\|^{q}-c_{2}\|u\|^{2^{*}} \\
& =\|u\|^{2}\left(\frac{p_{0}}{2}-c_{1}\|u\|^{q-2}-c_{2}\|u\|^{2^{*}-2}\right) \\
& \geq \sigma>0,
\end{aligned}
$$

desde que $\|u\|=\rho$, com $\rho>0$ suficientemente pequeno. Além disso, fixado $u \in H_{0}^{1}(\Omega) \backslash$ $\{0\}$, com $u \geq 0$, temos que

$$
I(t u)=\frac{t^{2}}{2}\|u\|_{p}^{2}-\frac{\lambda t^{q}}{q}|u|_{q}^{q}-\frac{t^{2^{*}}}{2^{*}}|u|_{2^{*}}^{2^{*}}
$$


e portanto $I(t u) \rightarrow-\infty$ quando $t \rightarrow+\infty$. Assim, considerando $e=t u \operatorname{com} t>0$ suficientemente grande, temos que $\|e\| \geq \rho$ e $I(e)<0$.

Vamos definir agora o nível minimax do Passo da Montanha

$$
c:=\inf _{\gamma \in \Gamma} \max _{t \in[0,1]} I(\gamma(t))
$$

em que

$$
\Gamma:=\left\{\gamma \in C\left([0,1], H_{0}^{1}(\Omega)\right): \gamma(0)=0, \gamma(1)=e\right\}
$$

Um argumento parecido ao usado no Lema 1.1 mostra que toda sequência $\left(u_{n}\right) \subset H_{0}^{1}(\Omega)$ tal que $I^{\prime}\left(u_{n}\right) \rightarrow 0$ e $I\left(u_{n}\right) \rightarrow c<\frac{1}{N}\left(p_{0} S\right)^{N / 2}$ possui subsequência convergente. Argumentando como em [35, Teorema 4.2] , podemos provar a seguinte caracterização do nível $\operatorname{minimax}$

$$
c=\inf _{u \in H_{0}^{1}(\Omega) \backslash\{0\}} \max _{t \geq 0} I(t u) .
$$

Assim, é suficiente provar que

Proposição 1.2. Existe $v \in H_{0}^{1}(\Omega) \backslash\{0\}$ tal que

$$
\sup _{t \geq 0} I(t v)<\frac{1}{N}\left(p_{0} S\right)^{N / 2}
$$

Demonstração: Seja $\psi \in C_{0}^{\infty}(\Omega)$ satisfazendo $0 \leq \psi \leq 1, \psi \equiv 1$ em $B(a, l), \psi \equiv 0$ em $\Omega \backslash \overline{B(a, 2 l)}$ e $l>0$ é tal que $\overline{B(a, 2 l)} \subset \Omega$. Para $\varepsilon>0$ definimos

$$
u_{\varepsilon}(x):=\frac{\psi(x)}{\left[\varepsilon+|x-a|^{2}\right]^{\frac{N-2}{2}}} \quad \text { e } \quad v_{\varepsilon}:=\frac{u_{\varepsilon}}{\left|u_{\varepsilon}\right|_{2^{*}}} .
$$

Consideremos a função

$$
h(t):=I\left(t v_{\varepsilon}\right), \quad t \geq 0,
$$

que é da forma $A t^{2}-B t^{q}-C t^{2^{*}}$, em que

$$
A:=\frac{1}{2}\left\|v_{\varepsilon}\right\|^{2}, \quad B:=\frac{\lambda}{q}\left|v_{\varepsilon}\right|_{q}^{q} \quad \text { e } \quad C:=\frac{1}{2^{*}}\left|v_{\varepsilon}\right|_{2^{*}}^{2^{*}}
$$

Observe que

$$
h^{\prime}(t)=t\left(2 A-q B t^{q-2}-2^{*} C t^{2^{*}-2}\right)
$$

e a função $t \mapsto\left(q B t^{q-2}+2^{*} C t^{2^{*}-2}\right)$ é crescente para $t>0$, já que $(q-2)>0$. Além disso, 
esta mesma função tende para infinto quando $t \rightarrow+\infty$. Portanto, existe um único $t>0$ tal que $2 A=q B t^{q-2}+2^{*} C t^{2^{*}-2}$, ou seja, tal que $h^{\prime}(t)=0$. Seja então $t_{\varepsilon}>0$ satisfazendo

$$
h^{\prime}\left(t_{\varepsilon}\right)=0
$$

Como $v_{\varepsilon} \geq 0$, temos ainda que

$$
0=h^{\prime}\left(t_{\varepsilon}\right)=t_{\varepsilon}\left\|v_{\varepsilon}\right\|_{p}^{2}-\lambda t_{\varepsilon}^{q-1}\left|v_{\varepsilon}\right|_{q}^{q}-t_{\varepsilon}^{2^{*}-1}\left|v_{\varepsilon}\right|_{2^{*}}^{2^{*}}
$$

Além disso, como $\left|v_{\varepsilon}\right|_{2^{*}}^{2^{*}}=1$ e $\lambda>0$, obtemos

$$
\left\|v_{\varepsilon}\right\|_{p}^{2}=\lambda t_{\varepsilon}^{q-2}\left|v_{\varepsilon}\right|_{q}^{q}+t_{\varepsilon}^{2^{*}-2} \geq t_{\varepsilon}^{2^{*}-2}
$$

Vamos tomar

$$
\widehat{t}:=\left\|v_{\varepsilon}\right\|_{p}^{\frac{2}{2^{*}-2}} \geq t_{\varepsilon}
$$

e considerar a função

$$
g(t):=\frac{1}{2} t^{2} \widehat{t}^{2^{*}-2}-\frac{1}{2^{*}} t^{2^{*}}, \quad t \geq 0 .
$$

Como $g^{\prime}(t)=t\left(\widehat{t}^{2^{*}-2}-t^{2^{*}-2}\right)$, a função $g$ é crescente no intervalo $[0, \widehat{t}]$. Além disso

$$
I\left(t_{\varepsilon} v_{\varepsilon}\right)=\frac{t_{\varepsilon}^{2}}{2}\left\|v_{\varepsilon}\right\|_{p}^{2}-\frac{\lambda}{q} t_{\varepsilon}^{q}\left|v_{\varepsilon}\right|_{q}^{q}-\frac{t_{\varepsilon}^{2^{*}}}{2^{*}}\left|v_{\varepsilon}\right|_{2^{*}}^{2^{*}}=g\left(t_{\varepsilon}\right)-\frac{\lambda}{q} t_{\varepsilon}^{q}\left|v_{\varepsilon}\right|_{q}^{q} \leq g(\widehat{t})-\frac{\lambda}{q} t_{\varepsilon}^{q}\left|v_{\varepsilon}\right|_{q}^{q},
$$

visto que $\left|v_{\varepsilon}\right|_{2^{*}}=1$, e $g$ é crescente em $[0, \widehat{t}]$. Como definimos $\widehat{t}=\left\|v_{\varepsilon}\right\|_{p}^{\frac{2}{2^{*}-2}}$, da definição de $g$

$$
g(\widehat{t})=\frac{1}{2} \widehat{t}^{2} \widehat{t}^{2^{*}-2}-\frac{1}{2^{*}} \widehat{t}^{2^{*}}=\left(\frac{1}{2}-\frac{1}{2^{*}}\right) \widehat{t}^{2^{*}}=\frac{1}{N}\left(\left\|v_{\varepsilon}\right\|_{p}^{2}\right)^{N / 2} .
$$

Portanto, temos que

$$
I\left(t_{\varepsilon} v_{\varepsilon}\right) \leq \frac{1}{N}\left(\left\|v_{\varepsilon}\right\|_{p}^{2}\right)^{N / 2}-\frac{t_{\varepsilon}^{q}}{q} \lambda\left|v_{\varepsilon}\right|_{q}^{q}
$$

Da definição de $u_{\varepsilon}$, segue que

$$
\left|u_{\varepsilon}\right|_{q}^{q}=\int \frac{\psi^{q}(x)}{\left[\varepsilon+|x-a|^{2}\right]^{\frac{q(N-2)}{2}}}=\int_{B(a, l)} \frac{1}{\left[\varepsilon+|x-a|^{2}\right]^{\frac{q(N-2)}{2}}} d x+O(1) .
$$


Agora, fazendo a mudança $x-a=\sqrt{\varepsilon} y$, temos que

$$
\begin{aligned}
\int_{B(a, l)} \frac{1}{\left[\varepsilon+|x-a|^{2}\right]^{\frac{q(N-2)}{2}}} d x & =\int_{B(l / \sqrt{\varepsilon}, 0)} \frac{\varepsilon^{\frac{N}{2}}}{\left[\varepsilon+\varepsilon|y|^{2}\right]^{\frac{q(N-2)}{2}}} d y \\
& =\varepsilon^{\frac{N}{2}-\frac{q(N-2)}{2}} \int_{B(l / \sqrt{\varepsilon}, 0)} \frac{1}{\left[1+|y|^{2}\right]^{\frac{q(N-2)}{2}}} d y .
\end{aligned}
$$

Notemos agora que

$$
\int_{B(l / \sqrt{\varepsilon}, 0)} \frac{1}{\left[1+|y|^{2}\right]^{\frac{q(N-2)}{2}}} d y \leq \int_{B(1,0)} \frac{1}{\left[1+|y|^{2}\right]^{\frac{q(N-2)}{2}}} d y+\int_{B(1,0)^{c}}|y|^{-q(N-2)} d y .
$$

Como $q>2$, temos que $-q(N-2)+N<0$, de modo que a última integral é finita. Assim

$$
\left|u_{\varepsilon}\right|_{q}^{q}=O\left(\varepsilon^{\frac{N}{2}-\frac{q(N-2)}{2}}\right)+O(1) .
$$

Por outro lado, uma estimativa devida a Brézis-Nirenberg (cf. [9, pg 444]) fornece

$$
\left|u_{\varepsilon}\right|_{2^{*}}^{2}=K_{2} \varepsilon^{-\frac{N-2}{2}}+O(\varepsilon)
$$

em que $K_{2}=K_{2}(N)>0$. Assim, pelo Teorema do Valor Médio, existe $\delta \in(0,1)$ tal que

$$
\begin{aligned}
\left|u_{\varepsilon}\right|_{2^{*}}^{q} & =\left(\left|u_{\varepsilon}\right|_{2^{*}}^{2}\right)^{\frac{q}{2}} \\
& =\left(K_{2} \varepsilon^{-\frac{(N-2)}{2}}+O(\varepsilon)\right)^{\frac{q}{2}} \\
& =K_{2}^{q / 2} \varepsilon^{-\frac{q(N-2)}{4}}+\frac{q}{2}\left(K_{2} \varepsilon^{-\frac{(N-2)}{2}}+\delta O(\varepsilon)\right)^{\frac{q}{2}-1} O(\varepsilon)
\end{aligned}
$$

portanto

$$
\left|u_{\varepsilon}\right|_{2^{*}}^{q}=O\left(\varepsilon^{-\frac{q(N-2)}{4}}\right)+O(1)
$$

Como $u_{\varepsilon}=\left|u_{\varepsilon}\right|_{2^{*}} v_{\varepsilon}$, e $Q_{0, p}\left(u_{\varepsilon}\right)=Q_{0, p}\left(\left|u_{\varepsilon}\right|_{2^{*}} v_{\varepsilon}\right)=\left\|v_{\varepsilon}\right\|_{p}^{2}$, segue do Lema 1.2 que

$$
\left\|v_{\varepsilon}\right\|_{p}^{2}= \begin{cases}p_{0} S+O(\varepsilon), & N \geq 5 \\ p_{0} S+O(\varepsilon|\log \varepsilon|), & N=4\end{cases}
$$

$\operatorname{logo}$ concluímos que $\left\|v_{\varepsilon}\right\|_{p}^{2}=p_{0} S+O(\varepsilon|\log \varepsilon|)$. Segue então do Teorema do Valor Médio que

$$
\left(\left\|v_{\varepsilon}\right\|_{p}^{2}\right)^{N / 2}=\left(p_{0} S\right)^{N / 2}+O(\varepsilon|\log \varepsilon|)
$$

Afirmamos que, para todo $\varepsilon>0$, existe um $C_{0}>0$ tal que $t_{\varepsilon}^{q} \geq q C_{0}$. Com efeito, 
suponhamos que para alguma sequência $\varepsilon_{n} \rightarrow 0^{+}$, temos $t_{\varepsilon_{n}} \rightarrow 0$. Teríamos, então,

$$
\left\|t_{\varepsilon_{n}} v_{\varepsilon_{n}}\right\|_{p}^{2}=t_{\varepsilon_{n}}^{2}\left\|v_{\varepsilon_{n}}\right\|_{p}^{2}=t_{\varepsilon_{n}}^{2}\left(p_{0} S+O\left(\varepsilon_{n}\left|\log \varepsilon_{n}\right|\right)\right)=o(1)
$$

e consequentemente $t_{\varepsilon_{n}} v_{\varepsilon_{n}} \rightarrow 0$ em $H_{0}^{1}(\Omega)$. Assim, usando a equivalência da definição do nível minimax $c$, a definição de $t_{\varepsilon}$ e a continuidade do funcional $I$ temos que

$$
0<c \leq \sup _{t \geq 0} I\left(t v_{\varepsilon_{n}}\right)=I\left(t_{\varepsilon_{n}} v_{\varepsilon_{n}}\right) \rightarrow I(0)=0
$$

o que é absurdo.

A limitação de $t_{\varepsilon}$ provada acima, (1.22) e (1.25) implicam que

$$
\begin{aligned}
I\left(t_{\varepsilon} v_{\varepsilon}\right) & \leq \frac{1}{N}\left(p_{0} S\right)^{\frac{N}{2}}+O(\varepsilon|\log \varepsilon|)-C_{0} \lambda\left|v_{\varepsilon}\right|_{q}^{q} \\
& =\frac{1}{N}\left(p_{0} S\right)^{\frac{N}{2}}+\varepsilon|\log \varepsilon|\left(O(1)-\lambda C_{0} \frac{\left|v_{\varepsilon}\right|_{q}^{q}}{\varepsilon|\log \varepsilon|}\right) .
\end{aligned}
$$

Para concluir a prova, basta mostrarmos que

$$
\lim _{\varepsilon \rightarrow 0^{+}} \frac{\left|v_{\varepsilon}\right|_{q}^{q}}{\varepsilon|\log \varepsilon|}=+\infty
$$

Por (1.23) e (2.9) temos que

$$
\begin{aligned}
\left|v_{\varepsilon}\right|_{q}^{q}=\frac{\left|u_{\varepsilon}\right|_{q}^{q}}{\left|u_{\varepsilon}\right|_{2^{*}}^{q}} & =\frac{O\left(\varepsilon^{\frac{N}{2}-\frac{q(N-2)}{2}}\right)+O(1)}{O\left(\varepsilon^{-\frac{q(N-2)}{4}}\right)+O(1)} \\
& =\frac{O\left(\varepsilon^{\frac{N}{2}-\frac{q(N-2)}{2}}\right) O\left(\varepsilon^{\frac{q(N-2)}{4}}\right)+O\left(\varepsilon^{\frac{q(N-2)}{4}}\right)}{1+O\left(\varepsilon^{\frac{q(N-2)}{4}}\right)} \\
& =\frac{O\left(\varepsilon^{\frac{N}{2}-\frac{q(N-2)}{4}}\right)}{1+O\left(\varepsilon^{\frac{q(N-2)}{4}}\right)}+O(1),
\end{aligned}
$$

ou ainda

$$
\frac{\left|v_{\varepsilon}\right|_{q}^{q}}{\varepsilon|\log \varepsilon|}=\frac{O\left(\varepsilon^{1-\frac{N}{2}+\frac{q(N-2)}{4}}|\log \varepsilon|\right)^{-1}}{1+O\left(\varepsilon^{\frac{q(N-2)}{4}}\right)}+O(1) .
$$

Como $q>2$, um cálculo imediato mostra que $1-\frac{N}{2}+\frac{q(N-2)}{4}>0$ e portanto (1.26) se verifica. A proposição está provada. 


\subsection{Estimativas do Lema 1.2}

Nessa seção final vamos apresentar a prova do Lema 1.2. Para tanto, vamos recordar que a função $u_{\varepsilon}$ é dada por

$$
u_{\varepsilon}(x):=\frac{\psi(x)}{\left[\varepsilon+|x-a|^{2}\right]^{(N-2) / 2}},
$$

em que $\varepsilon>0, \psi \in C_{0}^{\infty}(\Omega), 0 \leq \psi \leq 1, \psi \equiv 1$ em $B(a, l), \psi \equiv 0$ em $\Omega \backslash \overline{B(a, 2 l)}$ e $l>0$ é tal que $\overline{B(a, 2 l)} \subset \Omega$.

Segue das estimativas de Brézis-Nirenberg (cf. [9, pg 444]) que

$$
\left|u_{\varepsilon}\right|_{2^{*}}^{2}=\frac{K_{2}}{\varepsilon^{\frac{N-2}{2}}}+O(\varepsilon)
$$

$\mathrm{e}$

$$
\left|u_{\varepsilon}\right|_{2}^{2}= \begin{cases}\frac{K_{3}}{\varepsilon^{\frac{N-4}{2}}}+O(1), & N \geq 5 \\ \frac{\omega_{4}}{2}|\log \varepsilon|+O(1), & N=4\end{cases}
$$

em que

$$
K_{2}=\frac{1}{S}(N-2)^{2} \int_{\mathbb{R}^{N}} \frac{|y|^{2}}{\left[1+|y|^{2}\right]} d y, \quad K_{3}=\int_{\mathbb{R}^{N}} \frac{1}{\left[1+|y|^{2}\right]^{N-2}} d y
$$

e $\omega_{4}$ é a área da esfera unitária de $\mathbb{R}^{4}$.

No que segue vamos mostrar que, quando $\varepsilon \rightarrow 0^{+}$, valem as seguintes estimativas

$$
Q_{\lambda, p}\left(u_{\varepsilon}\right) \leq \begin{cases}p_{0} S-\lambda \frac{K_{3}}{K_{2}} \varepsilon+o(\varepsilon), & N \geq 5 \text { e } k>2 \\ p_{0} S-\left(\lambda-\frac{A_{2}}{K_{3}} \beta_{2}\right) \frac{K_{3}}{K_{2}} \varepsilon+o(\varepsilon), & N \geq 5 \text { e } k=2 ; \\ p_{0} S-\lambda \frac{\omega_{4}}{2 K_{2}} \varepsilon|\log \varepsilon|+o(\varepsilon|\log \varepsilon|), & N=4 \text { e } k>2 ; \\ p_{0} S-\left(\lambda-4 \beta_{2}\right) \frac{\omega_{4}}{2 K_{2}} \varepsilon|\log \varepsilon|+o(\varepsilon|\log \varepsilon|), & N=4 \text { e } k=2,\end{cases}
$$

em que $K_{2}, K_{3}$ e $A_{2}$ são como no enunciado do Lema 1.2 . 
A prova será feita em várias etapas. Iniciamos com um cálculo direto que mostra que

$$
\begin{aligned}
\left|\nabla u_{\varepsilon}(x)\right|^{2} & =\frac{\psi^{2}(x)|x-a|^{2}(N-2)^{2}}{\left[\varepsilon+|x-a|^{2}\right]^{N}}-\frac{2 \psi(x) \nabla \psi(x)(x-a)(N-2)}{\left[\varepsilon+|x-a|^{2}\right]^{N-1}} \\
& +\frac{|\nabla \psi(x)|^{2}}{\left[\varepsilon+|x-a|^{2}\right]^{N-2}} .
\end{aligned}
$$

De acordo com a igualdade acima, temos que

$$
\begin{aligned}
\int p(x)\left|\nabla u_{\varepsilon}(x)\right|^{2} & =\int_{\Omega \backslash B(a, l)} \frac{p(x)|\nabla \psi(x)|^{2}}{\left[\varepsilon+|x-a|^{2}\right]^{N-2}} d x \\
& +(N-2)^{2} \int \frac{p(x)|\psi(x)|^{2}|x-a|^{2}}{\left[\varepsilon+|x-a|^{2}\right]^{N}} \\
& -2(N-2) \int_{\Omega \backslash B(a, l)} \frac{p(x) \psi(x) \nabla \psi(x) \cdot(x-a)}{\left[\varepsilon+|x-a|^{2}\right]^{N-1}} d x,
\end{aligned}
$$

em que usamos o fato de que $\nabla \psi \equiv 0$ em $B(a, l)$.

Como as funções $p$ e $\psi$ são limitadas em $\bar{\Omega}$, segue do Teorema da Convergência Dominada que

$$
\int_{\Omega \backslash B(a, l)} \frac{p(x)|\nabla \psi(x)|^{2}}{\left[\varepsilon+|x-a|^{2}\right]^{N-2}} d x=O(1)
$$

e

$$
\int_{\Omega \backslash B(a, l)} \frac{p(x) \psi(x) \nabla \psi(x)(x-a)}{\left[\varepsilon+|x-a|^{2}\right]^{N-1}} d x=O(1),
$$

quando $\varepsilon \rightarrow 0$, de modo que

$$
\int p(x)\left|\nabla u_{\varepsilon}(x)\right|^{2}=(N-2)^{2} \int \frac{p(x)|\psi(x)|^{2}|x-a|^{2}}{\left[\varepsilon+|x-a|^{2}\right]^{N}}+O(1)
$$


Uma vez que $p(x)=p_{0}+\beta_{k}|x-a|^{k}+\theta(x)|x-a|^{k}$, obtemos

$$
\begin{aligned}
& \varepsilon^{\frac{N-2}{2}} \int p(x)\left|\nabla u_{\varepsilon}(x)\right|^{2} \\
& =(N-2)^{2} \varepsilon^{\frac{N-2}{2}}\left(\int \frac{p_{0}|\psi(x)|^{2}|x-a|^{2}}{\left[\varepsilon+|x-a|^{2}\right]^{N}}\right. \\
& \left.+\int \frac{|x-a|^{k+2}|\psi(x)|^{2}\left(\beta_{k}+\theta(x)\right)}{\left[\varepsilon+|x-a|^{2}\right]^{N}}\right)+O\left(\varepsilon^{\frac{N-2}{2}}\right) \\
& =(N-2)^{2} p_{0} \varepsilon^{\frac{N-2}{2}}\left(\int \frac{|x-a|^{2}}{\left[\varepsilon+|x-a|^{2}\right]^{N}}+\int \frac{|x-a|^{2}\left(|\psi(x)|^{2}-1\right)}{\left[\varepsilon+|x-a|^{2}\right]^{N}}\right) \\
& +(N-2)^{2} \varepsilon^{\frac{N-2}{2}} \int \frac{|x-a|^{k+2}\left(\beta_{k}+\theta(x)\right)}{\left[\varepsilon+|x-a|^{2}\right]^{N}} \\
& +(N-2)^{2} \varepsilon^{\frac{N-2}{2}} \int \frac{|x-a|^{k+2}\left(\beta_{k}+\theta(x)\right)\left(|\psi(x)|^{2}-1\right)}{\left[\varepsilon+|x-a|^{2}\right]^{N}}+O\left(\varepsilon^{\frac{N-2}{2}}\right) .
\end{aligned}
$$

Como $\left(|\psi(x)|^{2}-1\right) \equiv 0$ em $B(a, l)$, podemos proceder como antes e obter

$$
\begin{aligned}
\varepsilon^{\frac{N-2}{2}} \int p(x)\left|\nabla u_{\varepsilon}(x)\right|^{2} & =(N-2)^{2} p_{0} \varepsilon^{\frac{N-2}{2}} \int \frac{|x-a|^{2}}{\left[\varepsilon+|x-a|^{2}\right]^{N}} \\
& +(N-2)^{2} \varepsilon^{\frac{N-2}{2}} \int \frac{|x-a|^{k+2}\left(\beta_{k}+\theta(x)\right)}{\left[\varepsilon+|x-a|^{2}\right]^{N}} \\
& +O\left(\varepsilon^{\frac{N-2}{2}}\right) .
\end{aligned}
$$

Note que

$$
\int \frac{|x-a|^{2}}{\left[\varepsilon+|x-a|^{2}\right]^{N}}=\int_{\mathbb{R}^{N}} \frac{|x-a|^{2}}{\left[\varepsilon+|x-a|^{2}\right]^{N}} d x-\int_{\mathbb{R}^{N} \backslash \Omega} \frac{|x-a|^{2}}{\left[\varepsilon+|x-a|^{2}\right]^{N}} d x .
$$

Fazendo $x-a=\sqrt{\varepsilon} y$ e usando o Teorema da Mudança de Variável

$$
\int_{\mathbb{R}^{N}} \frac{|x-a|^{2}}{\left[\varepsilon+|x-a|^{2}\right]^{N}} d x=\varepsilon^{\frac{2-N}{2}} \int_{\mathbb{R}^{N}} \frac{|y|^{2}}{\left[1+|y|^{2}\right]^{N}} d y,
$$

em que a última integral é finita visto que $N>2$. Além disso, como $N>2$ e $a \in \Omega$,

$$
\int_{\mathbb{R}^{N} \backslash \Omega} \frac{|x-a|^{2}}{\left[\varepsilon+|x-a|^{2}\right]^{N}} d x=O(1) .
$$


Substituindo as últimas igualdades em (1.30) obtemos

$$
\begin{aligned}
\varepsilon^{\frac{N-2}{2}} \int p(x)\left|\nabla u_{\varepsilon}\right|^{2} & =p_{0} K_{1}+(N-2)^{2} \varepsilon^{\frac{N-2}{2}} \int \frac{|x-a|^{k+2}\left(\beta_{k}+\theta(x)\right)}{\left[\varepsilon+|x-a|^{2}\right]^{N}} \\
& +O\left(\varepsilon^{\frac{N-2}{2}}\right)
\end{aligned}
$$

em que

$$
K_{1}=(N-2)^{2} \int_{\mathbb{R}^{N}} \frac{|y|^{2}}{\left[1+|y|^{2}\right]^{N}} d y .
$$

Para a estimativa da integral que envolve o termo $|x-a|^{k+2}$ na estimativa acima, vamos considerar vários casos:

Caso 1: $N-2>k$.

Como $a \in \Omega$, temos que

$$
\begin{aligned}
\int \frac{|x-a|^{k+2}\left(\beta_{k}+\theta(x)\right)}{\left[\varepsilon+|x-a|^{2}\right]^{N}} & =\int_{\mathbb{R}^{N}} \frac{|x-a|^{k+2}\left(\beta_{k}+\theta(x)\right)}{\left[\varepsilon+|x-a|^{2}\right]^{N}} d x \\
& -\int_{\mathbb{R}^{N} \backslash \Omega} \frac{|x-a|^{k+2}\left(\beta_{k}+\theta(x)\right)}{\left[\varepsilon+|x-a|^{2}\right]^{N}} d x
\end{aligned}
$$

e portanto

$$
\int \frac{|x-a|^{k+2}\left(\beta_{k}+\theta(x)\right)}{\left[\varepsilon+|x-a|^{2}\right]^{N}}=\int_{\mathbb{R}^{N}} \frac{|x-a|^{k+2}\left(\beta_{k}+\theta(x)\right)}{\left[\varepsilon+|x-a|^{2}\right]^{N}} d x+O(1) .
$$

Fazendo $x-a=\sqrt{\varepsilon} y$ novamente, temos que

$$
\int_{\mathbb{R}^{N}} \frac{|x-a|^{k+2}\left(\beta_{k}+\theta(x)\right)}{\left[\varepsilon+|x-a|^{2}\right]^{N}} d x=\varepsilon^{\frac{k+2-N}{2}} \int_{\mathbb{R}^{N}} \frac{|y|^{k+2}\left(\beta_{k}+\theta(a+\sqrt{\varepsilon} y)\right)}{\left[1+|y|^{2}\right]^{N}} d y .
$$

Note agora que

$$
\int_{\mathbb{R}^{N}} \frac{|y|^{k+2}}{\left[1+|y|^{2}\right]^{N}}=\omega_{N} \int_{0}^{\infty} \frac{r^{k+2} r^{N-1}}{\left[1+r^{2}\right]^{N}} d r<+\infty
$$

visto que $N-2>k$. Assim, lembrando que $\lim _{x \rightarrow a} \theta(x)=0$, obtemos

$$
\int_{\mathbb{R}^{N}} \frac{|x-a|^{k+2}\left(\beta_{k}+\theta(x)\right)}{\left[\varepsilon+|x-a|^{2}\right]^{N}} d x=\varepsilon^{\frac{k+2-N}{2}}\left(\beta_{k} \int_{\mathbb{R}^{N}} \frac{|y|^{k+2}}{\left[1+|y|^{2}\right]^{N}} d y+o(1)\right) .
$$


Portanto, usando a igualdade acima e (1.32), podemos reescrever (1.31) como

$$
\varepsilon^{\frac{N-2}{2}} \int p(x)\left|\nabla u_{\varepsilon}\right|^{2}=p_{0} K_{1}+A_{k} \beta_{k} \varepsilon^{\frac{k}{2}}+O\left(\varepsilon^{\frac{N-2}{2}}\right),
$$

em que

$$
A_{k}=(N-2)^{2} \int_{\mathbb{R}^{N}} \frac{|y|^{k+2}}{\left[1+|y|^{2}\right]^{N}} d y .
$$

Como $k \geq 2$, devemos ter $N \geq 5$ e portanto temos dois subcasos a considerar:

Subcaso 1.1: $N \geq 5$ e $k=2$. (e adicionalmente $N-2>k$ )

Por (1.28) e (1.27) temos que

$$
Q_{\lambda, p}\left(u_{\varepsilon}\right) \leq \frac{\frac{p_{0} K_{1}+A_{2} \beta_{2} \varepsilon+O\left(\varepsilon^{\frac{N-2}{2}}\right)}{\varepsilon^{\frac{N-2}{2}}}-\lambda\left(\frac{K_{3}}{\varepsilon^{\frac{N-4}{2}}}+O(1)\right)}{\frac{K_{2}}{\varepsilon^{\frac{N-2}{2}}}+O(\varepsilon)}
$$

Observando que vale a igualdade

$$
\frac{1}{\frac{K_{2}}{\varepsilon^{\frac{N-2}{2}}}+O(\varepsilon)}=\frac{\varepsilon^{\frac{N-2}{2}}}{K_{2}+O\left(\varepsilon^{\frac{N}{2}}\right)}=\varepsilon^{\frac{N-2}{2}}\left(\frac{1}{K_{2}}+O\left(\varepsilon^{\frac{N}{2}}\right)\right),
$$

a desigualdade em (1.33) fica da seguinte forma

$$
\begin{aligned}
Q_{\lambda, p}\left(u_{\varepsilon}\right) & \leq \frac{\varepsilon^{\frac{N-2}{2}}}{K_{2}}\left(\frac{p_{0} K_{1}+A_{2} \beta_{2} \varepsilon+O\left(\varepsilon^{\frac{N-2}{2}}\right)}{\varepsilon^{\frac{N-2}{2}}}-\lambda\left(\frac{K_{3}}{\varepsilon^{\frac{N-4}{2}}}+O(1)\right)\right) \\
& +O\left(\varepsilon^{N-1}\right)\left(\frac{p_{0} K_{1}+A_{2} \beta_{2} \varepsilon+O\left(\varepsilon^{\frac{N-2}{2}}\right)}{\varepsilon^{\frac{N-2}{2}}}-\lambda\left(\frac{K_{3}}{\varepsilon^{\frac{N-4}{2}}}+O(1)\right)\right) \\
& =p_{0} \frac{K_{1}}{K_{2}}+\frac{A_{2}}{K_{2}} \beta_{2} \varepsilon-\lambda \frac{K_{3}}{K_{2}} \varepsilon+O\left(\varepsilon^{\frac{N-2}{2}}\right) \\
& =p_{0} S-\left(\lambda-\frac{A_{2}}{K_{3}} \beta_{2}\right) \frac{K_{3}}{K_{2}} \varepsilon+o(\varepsilon),
\end{aligned}
$$

visto que $\frac{K_{1}}{K_{2}}=S$.

Subcaso 1.2: $N \geq 5$ e $k>2$. (e adicionalmente $N-2>k$ ) 
De forma análoga ao que foi feito anteriormente,

$$
\begin{aligned}
Q_{\lambda, p}\left(u_{\varepsilon}\right) & \leq \frac{\frac{p_{0} K_{1}+A_{k} \beta_{k} \varepsilon^{\frac{k}{2}}+O\left(\varepsilon^{\frac{N-2}{2}}\right)}{\varepsilon^{\frac{N-2}{2}}}-\lambda\left(\frac{K_{3}}{\varepsilon^{\frac{N-4}{2}}}+O(1)\right)}{\frac{K_{2}}{\varepsilon^{\frac{N-2}{2}}}+O(\varepsilon)} \\
& =p_{0} \frac{K_{1}}{K_{2}}+\frac{A_{k}}{K_{2}} \beta_{k} \varepsilon^{\frac{k}{2}}-\lambda \frac{K_{3}}{K_{2}} \varepsilon+O\left(\varepsilon^{\frac{N-2}{2}}\right) \\
& =p_{0} S-\lambda \frac{K_{3}}{K_{2}} \varepsilon+o(\varepsilon) .
\end{aligned}
$$

Caso 2: $N-2<k$.

Nesse caso, vamos estimar a integral que envolve $|x-a|^{k+2}$ em (1.31) como segue: considere $R>0$ tal que $\Omega \subset B_{R}(a)$, de modo que

$$
\begin{aligned}
\int \frac{|x-a|^{k+2}\left(\beta_{k}+\theta(x)\right)}{\left[\varepsilon+|x-a|^{2}\right]^{N}} & =\int_{B(a, R)} \frac{|x-a|^{k+2}\left(\beta_{k}+\theta(x)\right)}{\left[\varepsilon+|x-a|^{2}\right]^{N}} d x \\
& -\int_{B(a, R) \backslash \Omega} \frac{|x-a|^{k+2}\left(\beta_{k}+\theta(x)\right)}{\left[\varepsilon+|x-a|^{2}\right]^{N}} d x \\
& =\int_{B(a, R)} \frac{|x-a|^{k+2}\left(\beta_{k}+\theta(x)\right)}{\left[\varepsilon+|x-a|^{2}\right]^{N}} d x+O(1) .
\end{aligned}
$$

Fazendo $y=x-a$ temos que

$$
\begin{aligned}
\int_{B(a, R)} \frac{|x-a|^{k+2}\left(\beta_{k}+\theta(x)\right)}{\left[\varepsilon+|x-a|^{2}\right]^{N}} d x & \leq\left(\beta_{k}+\|\theta\|_{\infty}\right) \int_{B(a, R)} \frac{|y|^{k+2}}{\left[\varepsilon+|y|^{2}\right]^{N}} d y \\
& =\left(\beta_{k}+\|\theta\|_{\infty}\right) \omega_{N} \int_{0}^{R} \frac{r^{k+2} r^{N-1}}{\left[\varepsilon+r^{2}\right]^{N}} d r .
\end{aligned}
$$

Como $N-2<k$, segue do Teorema da Convergência Dominada que

$$
\lim _{\varepsilon \rightarrow 0^{+}} \int_{0}^{R} \frac{r^{k+2} r^{N-1}}{\left[\varepsilon+r^{2}\right]^{N}} d r=\int_{0}^{R} r^{-N+k+1} d r<\infty,
$$

e portanto podemos reescrever (1.31) como

$$
\varepsilon^{\frac{N-2}{2}} \int p(x)\left|\nabla u_{\varepsilon}\right|^{2} \leq p_{0} S+O\left(\varepsilon^{\frac{N-2}{2}}\right) .
$$

Novamente, vamos considerar dois subcasos:

Subcaso 2.1: $N=4$ e $k>2$ (adicionalmente $N-2<k$ ) 
Por (1.28), (1.27) e analogamente ao caso anterior

$$
\begin{aligned}
Q_{\lambda, p}\left(u_{\varepsilon}\right) & \leq \frac{\frac{p_{0} K_{1}+O(\varepsilon)}{\varepsilon}-\lambda\left(\frac{\omega_{4}}{2}|\log \varepsilon|+O(1)\right)}{\frac{K_{2}}{\varepsilon}+O(\varepsilon)} \\
& =p_{0} S-\lambda \frac{\omega_{4}}{2 K_{2}} \varepsilon|\log \varepsilon|+O(\varepsilon) \\
& =p_{0} S-\lambda \frac{\omega_{4}}{2 K_{2}} \varepsilon|\log \varepsilon|+o(\varepsilon|\log \varepsilon|)
\end{aligned}
$$

Subcaso 2.2: $N \geq 5$ (e adicionalmente $N-2<k$ )

Analogamente ao que já foi feito, temos que

$$
\begin{aligned}
Q_{\lambda, p}\left(u_{\varepsilon}\right) & \leq \frac{\frac{p_{0} K_{1}+O\left(\varepsilon^{\frac{N-2}{2}}\right)}{\varepsilon^{\frac{N-2}{2}}}-\lambda\left(\frac{K_{3}}{\varepsilon^{\frac{N-4}{2}}}+O(1)\right)}{\frac{K_{2}}{\varepsilon^{\frac{N-2}{2}}}+O(\varepsilon)} \\
& =p_{0} \frac{K_{1}}{K_{2}}-\lambda \frac{K_{3}}{K_{2}} \varepsilon+O\left(\varepsilon^{\frac{N-2}{2}}\right) \\
& =p_{0} S-\lambda \frac{K_{3}}{K_{2}} \varepsilon+o(\varepsilon) .
\end{aligned}
$$

Caso 3: $k=N-2$ e $N \geq 5$.

Argumentando, como no caso anterior, temos que

$$
\begin{aligned}
\varepsilon^{\frac{N-2}{2}} \int p(x)\left|\nabla u_{\varepsilon}(x)\right|^{2} & \leq p_{0} K_{1} \\
& +(N-2)^{2}\left(\beta_{N-2}+\|\theta\|_{L^{\infty}}\right) \varepsilon^{\frac{N-2}{2}} \int_{B(a, R)} \frac{|x-a|^{N}}{\left[\varepsilon+|x-a|^{2}\right]^{N}} d x \\
& +O\left(\varepsilon^{\frac{N-2}{2}}\right) .
\end{aligned}
$$

Fazendo $y=x-a$ obtemos

$$
\int_{B(a, R)} \frac{|x-a|^{N}}{\left[\varepsilon+|x-a|^{2}\right]^{N}} d x=\omega_{N} \int_{0}^{R} \frac{r^{2 N-1}}{\left(\varepsilon+r^{2}\right)^{N}} d r .
$$


Observemos agora que, fazendo a mudança $t=\varepsilon+r^{2}$ e supondo $0<\varepsilon<1$, temos

$$
\begin{aligned}
\int_{0}^{R} \frac{r^{2 N-1}}{\left(\varepsilon+r^{2}\right)^{N}} d r & =\int_{0}^{R} \frac{r^{2(N-1)} r}{\left(\varepsilon+r^{2}\right)^{N}} d r=\frac{1}{2} \int_{\varepsilon}^{R^{2}+\varepsilon} \frac{(t-\varepsilon)^{N-1}}{t^{N}} d t \\
& =\frac{1}{2} \int_{\varepsilon}^{R^{2}+\varepsilon} \frac{t^{N-1}}{t^{N}} d t+\sum_{j=1}^{N-1} c_{j} \int_{\varepsilon}^{R^{2}+\varepsilon} \frac{t^{N-1-j} \varepsilon^{j}}{t^{N}} d t \\
& =\frac{1}{2} \log \left(R^{2}+\varepsilon\right)+\frac{1}{2}|\log \varepsilon|+\sum_{j=1}^{N-1} \varepsilon^{j} \widetilde{c}_{j}\left[\frac{\varepsilon^{j}}{\left(R^{2}+\varepsilon\right)^{j}}-1\right]
\end{aligned}
$$

Portanto, segue de (1.39) que

$$
\begin{aligned}
\varepsilon^{\frac{N-2}{2}} \int_{B(a, R)} \frac{|x-a|^{N}}{\left[\varepsilon+|x-a|^{2}\right]^{N}} d x & =\frac{\omega_{N}}{2} \varepsilon^{\frac{N-2}{2}} \log \left(R^{2}+\varepsilon\right)+\frac{\omega_{N}}{2} \varepsilon^{\frac{N-2}{2}}|\log \varepsilon| \\
& +\sum_{j=1}^{N-1} \widetilde{\widetilde{c}}_{j}\left[\frac{\varepsilon^{\frac{2 j+N-2}{2}}}{\left(R^{2}+\varepsilon\right)^{j}}-\varepsilon^{\frac{N-2}{2}}\right] \\
& =\frac{\omega_{N}}{2} \varepsilon^{\frac{N-2}{2}} \log \left(R^{2}+\varepsilon\right)+\frac{\omega_{N}}{2} \varepsilon^{\frac{N-2}{2}}|\log \varepsilon| \\
& +O\left(\varepsilon^{\frac{N}{2}}\right)+O\left(\varepsilon^{\frac{N}{2}+1}\right)+\cdots+O\left(\varepsilon^{\frac{N}{2}+N-2}\right)+O\left(\varepsilon^{\frac{N-2}{2}}\right) \\
& =\frac{\omega_{N}}{2} \varepsilon^{\frac{N-2}{2}}|\log \varepsilon|+O\left(\varepsilon^{\frac{N-2}{2}}\right),
\end{aligned}
$$

visto que $0<\varepsilon<1$. Logo, substituindo em (1.38), obtemos

$$
\begin{aligned}
\varepsilon^{\frac{N-2}{2}} \int p(x)\left|\nabla u_{\varepsilon}(x)\right|^{2} & \leq p_{0} K_{1} \\
& +\frac{(N-2)^{2}\left(\beta_{N-2}+\|\theta\|_{\infty}\right) \omega_{N}}{2} \varepsilon^{\frac{N-2}{2}}|\log \varepsilon|+O\left(\varepsilon^{\frac{N-2}{2}}\right) .
\end{aligned}
$$

Lembrando que $N \geq 5$ segue da desigualdade acima, (1.27) e (1.28) que

$$
\begin{aligned}
Q_{\lambda, p}\left(u_{\varepsilon}\right) & \leq \frac{\frac{p_{0} K_{1}+\frac{(N-2)^{2}\left(\beta_{k}+\|\theta\|_{L}\right) w_{N}}{2} \varepsilon^{\frac{N-2}{2}}|\log \varepsilon|+O\left(\varepsilon^{\frac{N-2}{2}}\right)}{\varepsilon^{\frac{N-2}{2}}}-\lambda\left(\frac{K_{3}}{\varepsilon^{\frac{N-4}{2}}}+O(1)\right)}{\frac{K_{2}}{\varepsilon^{\frac{N-2}{2}}}+O(\varepsilon)} \\
& =p_{0} \frac{K_{1}}{K_{2}}-\lambda \frac{K_{3}}{K_{2}} \varepsilon+\frac{(N-2)^{2}\left(\beta_{k}+\|\theta\|_{\infty}\right) \omega_{N}}{2 K_{2}} \varepsilon^{\frac{N-2}{2}}|\log \varepsilon| \\
& +O\left(\varepsilon^{\frac{N-2}{2}}\right)+O\left(\varepsilon^{\frac{N-2}{2}}|\log \varepsilon|\right) \\
& =p_{0} S-\lambda \frac{K_{1}}{K_{2}} \varepsilon+o(\varepsilon) .
\end{aligned}
$$

Caso 4: $N=4, k=2$. 
Nesse caso, consideraremos a hipótese adicional

$$
\int_{B(a, l)} \frac{\theta(x)}{|x-a|^{4}} d x<\infty .
$$

De acordo com a definião de $p(x)$ e (1.29), temos que

$$
\begin{aligned}
\int p(x)\left|\nabla u_{\varepsilon}(x)\right|^{2} & =\int \frac{p(x)|\nabla \psi(x)|^{2}}{\left[\varepsilon+|x-a|^{2}\right]^{2}}+4 \int \frac{p(x)|\psi(x)|^{2}|x-a|^{2}}{\left[\varepsilon+|x-a|^{2}\right]^{4}} \\
& -4 \int \frac{p(x) \psi(x) \nabla \psi(x) \cdot(x-a)}{\left[\varepsilon+|x-a|^{2}\right]^{3}} \\
& =4 p_{0} \int \frac{|\psi(x)|^{2}|x-a|^{2}}{\left[\varepsilon+|x-a|^{2}\right]^{4}} \\
& +4 \int \frac{|\psi(x)|^{2}|x-a|^{4}\left(\beta_{2}+\theta(x)\right)}{\left[\varepsilon+|x-a|^{2}\right]^{4}}+O(1) .
\end{aligned}
$$

Assim

$$
\begin{aligned}
& \int p(x)\left|\nabla u_{\varepsilon}(x)\right|^{2} \\
& =4 p_{0} \int_{\Omega \backslash B(a, l)} \frac{|\psi(x)|^{2}|x-a|^{2}}{\left[\varepsilon+|x-a|^{2}\right]^{4}} d x+4 p_{0} \int_{B(a, l)} \frac{|x-a|^{2}}{\left[\varepsilon+|x-a|^{2}\right]^{4}} d x \\
& +4 \int \frac{\left(\beta_{2}+\theta(x)\right)|\psi(x)|^{2}|x-a|^{4}}{\left[\varepsilon+|x-a|^{2}\right]^{4}}+O(1) \\
& =4 p_{0}\left[\int_{\mathbb{R}^{N}} \frac{|x-a|^{2}}{\left[\varepsilon+|x-a|^{2}\right]^{4}} d x-\int_{\mathbb{R}^{N} \backslash B(a, l)} \frac{|x-a|^{2}}{\left[\varepsilon+|x-a|^{2}\right]^{4}} d x\right] \\
& +4 \int \frac{\left(\beta_{2}+\theta(x)\right)|\psi(x)|^{2}|x-a|^{4}}{\left[\varepsilon+|x-a|^{2}\right]^{4}}+O(1) .
\end{aligned}
$$

Fazendo $x-a=\sqrt{\varepsilon} y$ e lembrando que $0 \leq \psi \leq 1$ e $N=4$ obtemos

$$
\begin{aligned}
\int p(x)\left|\nabla u_{\varepsilon}(x)\right|^{2} & \leq \frac{4 p_{0}}{\varepsilon} \int_{\mathbb{R}^{4}} \frac{|y|^{2}}{\left[1+|y|^{2}\right]^{4}} d y+4 \int \frac{\theta(x)|x-a|^{4}}{\left[\varepsilon+|x-a|^{2}\right]^{4}} \\
& +4 \beta_{2} \int \frac{|x-a|^{2}}{\left[\varepsilon+|x-a|^{2}\right]^{4}}+O(1) .
\end{aligned}
$$


Note que

$$
\int \frac{\theta(x)|x-a|^{4}}{\left[\varepsilon+|x-a|^{2}\right]^{4}}=O(1)+\int_{B(a, l)} \frac{\theta(x)}{|x-a|^{4}} d x
$$

e portanto concluímos de (1.41) que

$$
\int \frac{\theta(x)|x-a|^{4}}{\left[\varepsilon+|x-a|^{2}\right]^{4}}=O(1)
$$

Para estimar a última integral em (1.42) tomemos $R_{1}, R_{2}>0$ de tal modo que $\overline{B_{R_{1}}(a)} \subset \Omega \subset \overline{B_{R_{2}}(a)}$ e computemos

$$
\int_{B\left(a, R_{1}\right)} \frac{|x-a|^{4}}{\left[\varepsilon+|x-a|^{2}\right]} d x \leq \int \frac{|x-a|^{4}}{\left[\varepsilon+|x-a|^{2}\right]} \leq \int_{B\left(a, R_{2}\right)} \frac{|x-a|^{4}}{\left[\varepsilon+|x-a|^{2}\right]} d x .
$$

Observemos que, para todo $R>0$,

$$
\int_{B(a, R)} \frac{|x-a|^{4}}{\left[\varepsilon+|x-a|^{2}\right]} d x=\omega_{4} \int_{0}^{R} \frac{r^{4} r^{3}}{\left(\varepsilon+r^{2}\right)^{4}} d r .
$$

De forma semelhante ao que foi feito para o caso 3, teremos

$$
\begin{aligned}
\int_{0}^{R} \frac{r^{7}}{\left(\varepsilon+r^{2}\right)^{4}} d r & =\frac{\omega_{4}}{2}\left[\log \left(R^{2}+\varepsilon\right)+|\log \varepsilon|+3\left(\frac{\varepsilon}{R^{2}+\varepsilon}-1\right)\right. \\
& \left.-\frac{3}{2}\left(\frac{\varepsilon^{2}}{\left(R^{2}+\varepsilon\right)^{2}}-1\right)+\frac{1}{3}\left(\frac{\varepsilon^{3}}{\left(R^{2}+\varepsilon\right)^{3}}-1\right)\right] \\
& =\frac{\omega_{4}}{2}|\log \varepsilon|+O(1) .
\end{aligned}
$$

Portanto,

$$
\int p(x)\left|\nabla u_{\varepsilon}\right|^{2}=\frac{p_{0} K_{1}}{\varepsilon}+2 \beta_{2} \omega_{4}|\log \varepsilon|+O(1) .
$$

Nesse caso, da igualdade acima, segue que

$$
Q_{\lambda, p}\left(u_{\varepsilon}\right) \leq \frac{\frac{p_{0} K_{1}}{\varepsilon}+2 \beta_{2} \omega_{4}|\log \varepsilon|+O(1)-\lambda\left(\frac{\omega_{4}}{2}|\log \varepsilon|+O(1)\right)}{\frac{K_{2}}{\varepsilon}+O(\varepsilon)}
$$


$\log 0$

$$
\begin{aligned}
Q_{\lambda, p}\left(u_{\varepsilon}\right) & \leq p_{0} \frac{K_{1}}{K_{2}}+\frac{2 \beta_{2}}{K_{2}} \omega_{4} \varepsilon|\log \varepsilon|-\lambda \frac{\omega_{4}}{2 K_{2}} \varepsilon|\log \varepsilon|+O(\varepsilon)+o(\varepsilon|\log \varepsilon|) \\
& =p_{0} S-\frac{\omega_{4}}{2 K_{2}}\left(\lambda-4 \beta_{2}\right) \varepsilon|\log \varepsilon|+o(\varepsilon|\log \varepsilon|),
\end{aligned}
$$

e isto finaliza a prova lema. 


\section{CAPÍTULO 2}

\section{Perturbações do tipo côncavo-convexo}

Neste capítulo estudaremos a existência de solução positiva para o problema

$$
\left\{\begin{aligned}
-\operatorname{div}(p(x) \nabla u) & =b(x)|u|^{q-2} u+c(x)|u|^{r-2} u, & & x \in \Omega \\
u & =0, & & x \in \partial \Omega
\end{aligned}\right.
$$

em que $\Omega \subset \mathbb{R}^{N}$ é um domínio suave e limitado, $N \geq 4$ e $1<q<2<r \leq 2^{*}=2 N /(N-2)$. A função $p$ satisfaz as seguintes condições:

$\left(p_{1}\right) p \in H^{1}(\Omega) \cap C(\Omega)$

$\left(p_{2}\right)$ existe um ponto $a \in \Omega$ tal que

$$
p(a)=p_{0}:=\min \{p(x): x \in \bar{\Omega}\}>0
$$

$\left(p_{3}\right)$ existe $k>0, \beta_{k}>0$ e $\theta$ tais que, numa vizinhança de $a$, a função $p$ é da forma

$$
p(x)=p_{0}+\beta_{k}|x-a|^{k}+\theta(x)|x-a|^{k},
$$

$\operatorname{com} \lim _{x \rightarrow a} \theta(x)=0$.

Para $s>1$, denotamos por $s^{\prime}$ o expoente conjugado de $s$, a saber $s^{\prime}=s /(s-1)$. Os potenciais $b$ e $c$ satisfazem 
$\left(b_{1}\right) b \in L^{\sigma_{q}}(\Omega)$ para algum

$$
\left(\frac{r}{q}\right)^{\prime}<\sigma_{q} \leq\left(\frac{2}{q}\right)^{\prime}
$$

$\left(c_{1}\right) c \in L^{\infty}(\Omega), \operatorname{com} c \not \equiv 0$

$\left(b c_{1}\right)$ existe $\delta>0$ tal que $B_{\delta}(a) \subset\left(\Omega_{b}^{+} \cap \Omega_{c}^{+}\right)$e

$$
|c|_{\infty}-c(x) \leq M|x-a|^{\gamma}
$$

q.t.p. em $B_{\delta}(a)$, em que $\Omega_{b}^{+}:=\{x \in \Omega: b(x)>0\}, \Omega_{c}^{+}:=\{x \in \Omega: c(x)>0\}$, $M>0$ e $\gamma>(N-2) / 2$.

Soluções fracas não-negativas do problema $(P C)$ são pontos críticos do funcional $I \in$ $C^{1}\left(H_{0}^{1}(\Omega), \mathbb{R}\right)$ definido por

$$
I(u)=\frac{1}{2} \int p(x)|\nabla u|^{2}-\frac{1}{q} \int b(x)\left(u^{+}\right)^{q}-\frac{1}{r} \int c(x)\left(u^{+}\right)^{r} .
$$

Estamos então em condições de enunciar os dois principais resultados deste capítulo, quais sejam:

Teorema 2.1. Suponha que $1<q<2<r<2^{*}$, a função $p \in H^{1}(\Omega) \cap C(\bar{\Omega})$ satisfaz $\left(p_{1}\right)-\left(p_{3}\right)$ com $k>2$, as funções b e c satisfazem $\left(b_{1}\right)$ e $\left(c_{1}\right)$. Se $|b|_{\sigma_{q}}$ é suficientemente pequeno, então o problema (PC) tem pelos menos duas soluções não negativas $u_{0} e u_{1}$ satisfazendo $I\left(u_{0}\right)<0<I\left(u_{1}\right)$.

Teorema 2.2. Suponha que $r=2^{*}, p \in H^{1}(\Omega) \cap C(\bar{\Omega})$ satisfaz $\left(p_{1}\right)-\left(p_{3}\right)$ com $N<(2 k+$ $2)$, as funções b e c satisfazem $\left(b_{1}\right),\left(c_{1}\right)$, e $\left(b c_{1}\right)$. Se $|b|_{\sigma_{q}}$ é suficientemente pequeno, então o problema $(P C)$ tem pelos menos duas soluções não negativas $u_{0} e u_{1}$, com $I\left(u_{0}\right)<0$ e $u_{1} \neq 0$.

\subsection{Lemas auxiliares}

Nesta seção serão enunciados e demonstrados três lemas que fornecem as propriedades geométricas do funcional $I$.

Lema 2.1. Existem $\rho, \alpha>0$ tais que $I(u) \geq \alpha>0$, para todo $u \in H_{0}^{1}(\Omega)$ satisfazendo $\|u\|=\rho$, desde que a função b satisfaça $\left(b_{1}\right)$ e $|b|_{\sigma_{q}}$ seja suficientemente pequeno. 
Demonstração: Pela desigualdade de Hölder, temos que

$$
\left|\int b(x)\left(u^{+}\right)^{q}\right| \leq|b|_{\sigma_{q}}\left(\int|u|^{q \sigma_{q}^{\prime}}\right)^{1 / \sigma_{q}^{\prime}} .
$$

Vamos definir, para cada $\tau \in\left[2,2^{*}\right]$, a constante positiva

$$
S_{\tau}:=\inf \left\{\int|\nabla u|^{2}: \int|u|^{\tau}=1\right\}<+\infty
$$

Vale ressaltar que, quando $\tau=2^{*}$, usaremos a notação usual $S:=S_{2^{*}}$. Assim, vale a seguinte desigualdade

$$
S_{\tau} \leq \frac{\|u\|^{2}}{|u|_{\tau}^{2}}
$$

Da desigualdade acima e da definição de $p(x)$, segue que

$$
\begin{aligned}
I(u) & =\frac{1}{2} \int p(x)|\nabla u|^{2}-\frac{1}{q} \int b(x)\left(u^{+}\right)^{q}-\frac{1}{r} \int c(x)\left(u^{+}\right)^{r} \\
& \geq \frac{p_{0}}{2}\|u\|^{2}-\frac{1}{q}|b|_{\sigma_{q}}|u|_{q \sigma_{q}^{\prime}}^{q}-\frac{1}{r}|c|_{\infty}|u|_{r}^{r} \\
& \geq p_{0} \frac{\|u\|^{q}}{2}\left\{\|u\|^{2-q}-\frac{2}{r p_{0}}|c|_{\infty} S_{r}^{-r / 2}\|u\|^{r-q}-\frac{2}{q p_{0}}|b|_{\sigma_{q}} S_{q \sigma_{q}^{\prime}}^{-q / 2}\right\} .
\end{aligned}
$$

Definindo $B:=2\left(r p_{0}\right)^{-1}|c|_{\infty} S_{r}^{-r / 2}$, por um cálculo direto, a função $f:(0,+\infty) \rightarrow \mathbb{R}$, dada por

$$
f(t):=t^{2-q}-B t^{r-q}
$$

atinge máximo no ponto

$$
t_{0}:=\left[\frac{(2-q)}{B(r-q)}\right]^{1 /(r-2)}>0
$$

Para $M:=f\left(t_{0}\right)$ e $\|u\|=t_{0}$, segue que

$$
I(u) \geq p_{0} \frac{t_{0}^{q}}{2}\left\{M-\frac{2}{q p_{0}}|b|_{\sigma_{q}} S_{q \sigma_{q}^{\prime}}^{-q / 2}\right\} \geq \frac{t_{0}^{q}}{2} \frac{M}{2}=\alpha>0,
$$

sempre que

$$
|b|_{\sigma_{q}} \leq M q p_{0} S_{q \sigma_{q}^{\prime}}^{-q / 2} / 4 .
$$

Fazendo $t_{0}=\rho$, o resultado segue.

O próximo lema é fundamental para assegurar a existência de uma solução com energia 
negativa.

Lema 2.2. Se b satisfaz $\left(b_{1}\right),\left(b c_{1}\right)$ e (2.1) e $\rho>0$ é como no Lema 2.1, então

$$
-\infty<I_{0}:=\inf _{u \in \overline{B_{\rho}(0)}} I(u)<0
$$

é atingido para algum $u_{0} \in B_{\rho}(0)$.

Demonstração: Para garantir que $I_{0}>-\infty$, basta observar que, se $\|u\| \leq M_{0}$, então

$$
\begin{aligned}
|I(u)| & \leq \frac{1}{2}\|u\|_{p}^{2}+\frac{1}{q}\left|\int b(x)\left(u^{+}\right)^{q}\right|+\frac{1}{r}\left|\int c(x)\left(u^{+}\right)^{r}\right| \\
& \leq \frac{1}{2}|p|_{\infty}\|u\|^{2}+\frac{1}{q}|b|_{\sigma_{q}} S_{q \sigma_{q}^{\prime}}^{-q / 2}\|u\|^{q}+\frac{1}{r}|c|_{\infty} S_{r}^{-r / 2}\|u\|^{r} \\
& \leq C\left(M_{0}^{2}+M_{0}^{q}+M_{0}^{r}\right),
\end{aligned}
$$

em que

$$
C=\max \left\{\frac{1}{2}|p|_{\infty}, \frac{1}{q}|b|_{\sigma_{q}} S_{q \sigma_{q}^{\prime}}^{-q / 2}, \frac{1}{r}|c|_{\infty} S_{r}^{-r / 2}\right\} .
$$

Portanto

$$
\inf _{u \in B_{M_{0}}(0)}|I(u)|<+\infty .
$$

Seja $\delta>0$ como na hipótese $\left(b c_{1}\right)$. Como $B_{\delta}(a) \subset \Omega_{b}^{+}$, existe $\varphi \in C_{0}^{\infty}\left(B_{\delta}(a)\right)$ tal que $\int b(x) \varphi^{q}>0$. Assim

$$
\frac{I(t \varphi)}{t^{q}} \leq \frac{t^{2-q}}{2}\|\varphi\|_{p}^{2}-\frac{1}{q} \int b(x) \varphi^{q}-\frac{t^{r-q}}{r} \int c(x) \varphi^{r}
$$

e como $(2-q)>0$ e $(r-q)>0$, obtemos

$$
\lim _{t \rightarrow 0^{+}} \sup \frac{I(t \varphi)}{t^{q}} \leq-\frac{1}{q} \int b(x) \varphi^{q}<0 .
$$

Logo, $I(t \varphi)<0$ para $t>0$ pequeno. Com isso, concluímos que $I_{0}<0$.

Seja agora $\left(u_{n}\right) \subset \bar{B}_{\rho}(0)$ uma sequência minimizante para $I_{0}$. Pelo Princípio Variacional de Ekeland, podemos assumir que, a menos de subsequência,

$$
I\left(u_{n}\right) \rightarrow I_{0} \quad \text { e } \quad I^{\prime}\left(u_{n}\right) \rightarrow 0
$$


Como $\left(u_{n}\right)$ é limitada e $2 \leq q \sigma_{q}^{\prime}<2^{*}$ então, a menos de subsequência,

$$
\begin{aligned}
u_{n} & \rightarrow u_{0} \quad \text { fracamente em } \quad H_{0}^{1}(\Omega), \\
u_{n} & \rightarrow u_{0} \quad \text { fortemente em } \quad L^{q \sigma_{q}^{\prime}}(\Omega), \\
u_{n}^{+}(x) & \rightarrow u_{0}^{+}(x),\left|u_{n}(x)\right| \leq \psi(x) \quad \text { q.t.p. em } \quad \Omega,
\end{aligned}
$$

para alguma $\psi \in L^{q \sigma_{q}^{\prime}}(\Omega)$. Portanto, q.t.p. em $\Omega$, vale

$$
\left|b(x)\left(u_{n}^{+}\right)^{q}\right| \leq \frac{1}{\sigma_{q}} b(x)^{\sigma_{q}}+\frac{1}{\sigma_{q}^{\prime}} \psi(x)^{q \sigma_{q}^{\prime}}
$$

em que usamos a última estimativa de (2.2) e a desigualdade de Young. Como $\psi \in L^{q \sigma_{q}^{\prime}}(\Omega)$ e $b \in L^{\sigma_{q}}(\Omega)$, o lado direito da equação acima pertence a $L^{1}(\Omega)$. Portanto, do Teorema da Convergência Limitada de Lebesgue e de (2.2), segue que

$$
\lim _{n \rightarrow \infty} \int b(x)\left(u_{n}^{+}\right)^{q}=\int b(x)\left(u_{0}^{+}\right)^{q} .
$$

Tomaremos como verdadeiro o fato $I^{\prime}\left(u_{0}\right)=0$, que será provado ao final da demonstração. Feito isso, da convergência acima e da convergência fraca de $\left(u_{n}\right)$ temos que

$$
\begin{aligned}
I_{0} & =\lim _{n \rightarrow \infty} \inf \left(I\left(u_{n}\right)-\frac{1}{r} I^{\prime}\left(u_{n}\right) u_{n}\right) \\
& =\lim _{n \rightarrow \infty} \inf \left\{\left(\frac{1}{2}-\frac{1}{r}\right)\left\|u_{n}\right\|_{p}^{2}+\left(\frac{1}{r}-\frac{1}{q}\right) \int b(x)\left(u_{n}^{+}\right)^{q}\right\} \\
& \geq\left(\frac{1}{2}-\frac{1}{r}\right)\left\|u_{0}\right\|_{p}^{2}-\frac{1}{q} \int b(x)\left(u_{0}^{+}\right)^{q} \\
& =I\left(u_{0}\right)-\frac{1}{r} I^{\prime}\left(u_{0}\right)=I\left(u_{0}\right),
\end{aligned}
$$

e portanto $I\left(u_{0}\right) \leq I_{0}<0$. Mas pelo Lema 2.1, $u_{0}$ não pertence ao conjunto $\partial B_{\rho}(0)$, e isso conclui o resultado.

Resta-nos mostrar que $I^{\prime}\left(u_{0}\right)=0$. Seja $\varphi \in C_{0}^{\infty}(\Omega)$ e definamos $A:=\operatorname{supp}(\varphi)$ o suporte da função $\varphi$. Como $\sigma_{q}>(r / q)^{\prime}=r /(r-q)$, podemos escolher $q_{0} \in(2, r)$ tal que

$$
\sigma_{q}>\frac{q_{0}}{q_{0}-q}>\frac{q_{0}}{\left(q_{0}+1\right)-q}
$$

Assim, temos que

$$
\frac{1}{\sigma_{q}}+\frac{q-1}{q_{0}}<\frac{\left(q_{0}+1\right)-q}{q_{0}}+\frac{q-1}{q_{0}}=1
$$


Logo, existe $\theta>0$ satisfazendo

$$
\frac{1}{\sigma_{q}}+\frac{1}{q_{0} /(q-1)}+\frac{1}{\theta}=1
$$

Usando a desigualdade de Young, existe $C>0$ tal que

$$
\begin{aligned}
\left|b(x)\left(u_{n}^{+}\right)^{q-1} \varphi\right| & \leq C\left(|b(x)|^{\sigma_{q}}+\left|u_{n}(x)\right|^{q_{0}}+|\varphi|^{\theta}\right) \\
& \leq C\left(|a(x)|^{\sigma_{q}}+\left|\psi_{q_{0}}(x)\right|^{q_{0}}+|\varphi|^{\theta}\right)
\end{aligned}
$$

q.t.p. em $A$. Como $\psi$ e regular, o lado direito da desigualdade acima pertence à $L^{1}(A)$. Pelo Teorema da Convergência Dominada de Lebesgue, segue que

$$
\lim _{n \rightarrow+\infty} \int b(x)\left(u_{n}^{+}\right)^{q-1} \varphi=\int b(x)\left(u_{0}^{+}\right)^{q-1} \varphi .
$$

Além disso, como $c \in L^{\infty}(\Omega)$, é fácil ver que

$$
\lim _{n \rightarrow+\infty} \int c(x)\left(u_{n}^{+}\right)^{r-1} \varphi=\int c(x)\left(u_{0}^{+}\right)^{r-1} \varphi
$$

Com as duas convergências acima, concluímos que

$$
0=\lim _{n \rightarrow+\infty} I^{\prime}\left(u_{n}\right) \varphi=I^{\prime}\left(u_{0}\right) \varphi
$$

para todo $\varphi \in C_{0}^{\infty}(\Omega)$. Assim, a afirmação segue por densidade, o que completa a prova do lema.

Observação: A função $u_{0}$ acima satisfaz $u_{0} \geq 0 \mathrm{em} \Omega$. De fato, se tomarmos $v=u_{0}^{-}$ como função teste, teremos

$$
0=I^{\prime}\left(u_{0}\right) u_{0}^{-}=\int p(x)\left(\nabla u_{0} \cdot \nabla u_{0}^{-}\right)-\int b(x)\left(u_{0}^{+}\right)^{q-1} u_{0}^{-}-\int c(x)\left(u_{0}^{+}\right)^{r-1} u_{0}^{-}
$$

mostrando que $\left\|u_{0}^{-}\right\|_{p}^{2}=0$, ou seja, $u_{0} \geq 0$ em $\Omega$.

Lema 2.3. Se $b$ e c satisfazem $\left(b_{1}\right),\left(c_{1}\right)$ e $\left(b c_{1}\right), u_{0}$ é como no lema anterior e $\varphi \in$ $C_{0}^{\infty}\left(B_{\delta}(a)\right) \backslash\{0\}$ é não negativa, então

$$
\lim _{t \rightarrow+\infty} I\left(u_{0}+t \varphi\right)=-\infty .
$$


Demonstração: Como $u_{0} \geq 0$ em $\Omega$ e $B_{\delta}(a) \subset\left(\Omega_{b}^{+} \cap \Omega_{c}^{+}\right)$,

$$
\int_{B_{\delta}(a)} b(x)\left(u_{0}+t \varphi\right)^{q} \geq \int_{B_{\delta}(a)} b(x)(t \varphi)^{q} .
$$

Logo, lembrando que $\varphi \equiv 0$ fora de $B_{\delta}(a)$, temos

$$
\begin{aligned}
\int b(x)\left(u_{0}+t \varphi\right)^{q} & =\int_{B_{\delta}(a)} b(x)\left(u_{0}+t \varphi\right)^{q}+\int_{\Omega \backslash B_{\delta}(a)} b(x)\left(u_{0}+t \varphi\right)^{q} \\
& \geq \int_{B_{\delta}(a)} b(x)(t \varphi)^{q}+\int_{\Omega \backslash B_{\delta}(a)} b(x) u_{0}^{q} .
\end{aligned}
$$

Analogamente

$$
\int c(x)\left(u_{0}+t \varphi\right)^{r} \geq \int_{B_{\delta}(a)} c(x)(t \varphi)^{r}+\int_{\Omega \backslash B_{\delta}(a)} c(x) u_{0}^{r} .
$$

Portanto, das desigualdades acima e da definição de $I$, segue que

$$
\begin{aligned}
I\left(u_{0}+t \varphi\right) & =\frac{1}{2} \int p(x)\left|\nabla\left(u_{0}+t \varphi\right)\right|^{2}-\frac{1}{q} \int b(x)\left(u_{0}+t \varphi\right)^{q}-\frac{1}{r} \int\left(u_{0}+t \varphi\right)^{r} \\
& \leq \frac{1}{2} \int p(x)\left|\nabla u_{0}\right|^{2}+t \int p(x)\left(\nabla u_{0} \cdot \nabla \varphi\right)+\frac{t^{2}}{2}\|\varphi\|_{p}^{2}-\frac{1}{q} \int_{B_{\delta}(a)} b(x)(t \varphi)^{q} \\
& -\frac{1}{q} \int_{\Omega \backslash B_{\delta}(a)} b(x) u_{0}^{q}-\frac{1}{r} \int_{B_{\delta}(a)} c(x)(t \varphi)^{r}-\frac{1}{r} \int_{\Omega \backslash B_{\delta}(a)} c(x) u_{0}^{r} \\
& \leq O(1)+O(t)+O\left(t^{2}\right)-\frac{t^{q}}{q} \int_{B_{\delta}(a)} b(x) \varphi^{q}-\frac{t^{r}}{r} \int_{B_{\delta}(a)} c(x) \varphi^{r}
\end{aligned}
$$

quando $t \rightarrow+\infty$. Como $q<2<r$, e as últimas integrais são positivas, então

$$
\lim _{t \rightarrow+\infty} I\left(u_{0}+t \varphi\right)=-\infty
$$

como queríamos demonstrar.

\subsection{Prova do Teorema 2.1}

Antes da prova do Teorema 2.1, vamos provar que $I$ satisfaz Palais-Smale no caso subcrítico.

Lema 2.4. Se $2<r<2^{*}$, então I satisfaz $(P S)_{d}$ para todo $d \in \mathbb{R}$. 
Demonstração: Como $I^{\prime}\left(u_{n}\right) \rightarrow 0$, para $n$ suficientemente grande, $\left\|I^{\prime}\left(u_{n}\right)\right\| \leq r$. Logo

$$
\left|I^{\prime}\left(u_{n}\right) u_{n}\right| \leq\left\|I^{\prime}\left(u_{n}\right)\right\|\left\|u_{n}\right\| \leq r\left\|u_{n}\right\|
$$

e consequentemente $-(1 / r) I^{\prime}\left(u_{n}\right) u_{n} \leq\left\|u_{n}\right\|$. Usando o fato de que $I\left(u_{n}\right)=d+o(1)$ e a desigualdade acima, temos que

$$
\begin{aligned}
d+\left\|u_{n}\right\|+o(1) & \geq I\left(u_{n}\right)-\frac{1}{r} I^{\prime}\left(u_{n}\right) u_{n} \\
& =\frac{1}{2} \int p(x)\left|\nabla u_{n}\right|^{2}-\frac{1}{q} \int b(x)\left(u_{n}^{+}\right)^{q}-\frac{1}{r} \int c(x)\left(u_{n}^{+}\right)^{r} \\
& -\frac{1}{r} \int p(x)\left|\nabla u_{n}\right|^{2}+\frac{1}{r} \int b(x)\left(u_{n}^{+}\right)^{q}+\frac{1}{r} \int c(x)\left(u_{n}^{+}\right)^{r},
\end{aligned}
$$

e portanto

$$
d+\left\|u_{n}\right\|+o(1) \geq\left(\frac{1}{2}-\frac{1}{r}\right)\left\|u_{n}\right\|_{p}^{2}-\left(\frac{1}{q}-\frac{1}{r}\right) \int b(x)\left(u_{n}^{+}\right)^{q}
$$

Pela desigualdade de Hölder temos que

$$
\int b(x)\left(u_{n}^{+}\right)^{q} \leq|b|_{\sigma_{q}}\left(\int\left|u_{n}\right|^{q \sigma_{q}^{\prime}}\right)^{1 / \sigma_{q}^{\prime}} .
$$

Além disso, como $H_{0}^{1}(\Omega) \hookrightarrow L^{\tau}(\Omega)$, para $2 \leq \tau \leq 2^{*}$, existe $C_{1}>0$ tal que

$$
\left(\int\left|u_{n}\right|^{q \sigma_{q}^{\prime}}\right)^{\frac{1}{q \sigma_{q}^{\prime}}} \leq C_{1}\left\|u_{n}\right\|
$$

Substituindo as desigualdades acima em (2.3), concluímos que

$$
d+\left\|u_{n}\right\|+o(1) \geq p_{0}\left(\frac{1}{2}-\frac{1}{r}\right)\left\|u_{n}\right\|^{2}-\left(\frac{1}{q}-\frac{1}{r}\right) C_{1}\left\|u_{n}\right\|^{q} .
$$

Tendo em vista que $(1 / q-1 / r)>0$ e $(1 / 2-1 / r)>0$ e que $q<2<r$, garantimos que $\left(u_{n}\right)$ e limitada em $H_{0}^{1}(\Omega)$.

Portanto, a menos de subsequência,

$$
\begin{aligned}
& u_{n} \rightarrow u \quad \text { em } \quad H_{0}^{1}(\Omega), \\
& u_{n} \rightarrow u \quad \text { em } \quad L^{s}(\Omega)
\end{aligned}
$$

para qualquer $2 \leq s \leq p<r$. Da maneira que foi definido $\sigma_{q}$, existe $2 \leq r_{0}<r$ tal que 
$\sigma_{q}=\left(r_{0} / q\right)^{\prime}=r_{0} /\left(r_{0}-q\right)$. Assim

$$
\frac{1}{\sigma_{q}}+\frac{1}{r_{0}(q-1)}+\frac{1}{r_{0}}=\frac{r_{0}-q}{r_{0}}+\frac{q-1}{r_{0}}+\frac{1}{r_{0}}=1 .
$$

Usando a desigualdade de Hölder

$$
\left|\int b(x)\left(u_{n}^{+}\right)^{q-1}\left(u_{n}-u\right)\right| \leq\|b\|_{\sigma_{q}}\left\|u_{n}\right\|_{r_{0}}^{q-1}\left\|u_{n}-u\right\|_{r_{0}}=o(1) .
$$

Novamente por Hölder

$$
\left|\int c(x)\left(u_{n}^{+}\right)^{r-1}\left(u_{n}-u\right)\right| \leq\|c\|_{\infty}\left\|u_{n}\right\|_{r}^{r-1}\left\|u_{n}-u\right\|_{r}=o(1) .
$$

Portanto, das duas últimas convergências,

$$
o(1)=I^{\prime}\left(u_{n}\right)\left(u_{n}-u\right)=\left\|u_{n}\right\|_{p}^{2}-\|u\|_{p}^{2}+o(1),
$$

quanto $n \rightarrow+\infty$. Como $u_{n} \rightarrow u$ e $\left\|u_{n}\right\|_{p} \rightarrow\|u\|_{p}$ e as normas $\|\cdot\|_{p}$ e $\|\cdot\|$ são equivalentes, concluímos que $u_{n} \rightarrow u$ fortemente em $H_{0}^{1}(\Omega)$, desse modo, finalizamos a prova.

Observação: Vale notar que a prova da limitação da sequência (PS) acima permanece válida no caso crítico $r=2^{*}$.

Estamos portanto em condições de demonstrar o primeiro teorema deste capítulo.

Demonstração do Teorema 2.1: No Lema 2.2, sob as condições $\left(b_{1}\right),\left(b c_{1}\right)$ e o fato de $|b|_{\sigma_{q}}$ ser suficientemente pequeno, provamos a existência de uma solução $u_{0} \in H_{0}^{1}(\Omega)$ que satisfaz $I\left(u_{0}\right)<0$. Para obter a segunda solução com energia positiva, vamos considerar $\rho>0$ determinado no Lema 2.1 e a função $\varphi$ do Lema 2.3. Seja $t_{0}>0$ suficientemente grande tal que $e:=u_{0}+t_{0} \varphi$ satisfaz $I(e) \leq I\left(u_{0}\right)$. Vamos definir

$$
\widetilde{d}:=\inf _{\gamma \in \Gamma} \max _{t \in[0,1]} I(\gamma(t))
$$

em que

$$
\Gamma:=\left\{\gamma \in C\left([0,1], H_{0}^{1}(\Omega)\right): \gamma(0)=u_{0}, \gamma(1)=e\right\} .
$$

Pela definição de $\widetilde{d}$ e pelo Lema 2.1, garantimos que $\widetilde{d} \geq \alpha>0$. Assim, pelo Teorema do Passo da Montanha, existe uma sequência $\left(u_{n}\right) \subset H_{0}^{1}(\Omega)$ satisfazendo $I\left(u_{n}\right) \rightarrow \widetilde{d}$ e $I^{\prime}\left(u_{n}\right) \rightarrow 0$. Mas pelo Lema $2.4,\left(u_{n}\right)$ satisfaz $(P S)_{\widetilde{d}}$, ou seja, a menos de subsequência, 
$u_{n} \rightarrow u_{1}$ em $H_{0}^{1}(\Omega)$ e além disso $u_{1}$ é tal que $I\left(u_{1}\right)=\widetilde{d}>0$ e $I^{\prime}\left(u_{1}\right)=0$, e isso garante a segunda solução com energia positiva.

\subsection{Prova do Teorema 2.2}

No que se segue, denotaremos por $u_{0}$ a solução com energia negativa obtida no Lema 2.2. Para estudar o caso crítico, como não existe compacidade na imersão $H_{0}^{1}(\Omega) \hookrightarrow$ $L^{2^{*}}(\Omega)$, enunciamos o seguinte lema:

Lema 2.5. Se I tem apenas $u_{0}$ e zero como pontos críticos, então I satisfaz $(P S)_{d}$ para todo

$$
d<d^{*}:=I\left(u_{0}\right)+\frac{1}{N} \frac{\left(p_{0} S\right)^{N / 2}}{|c|_{\infty}^{(N-2) / 2}} .
$$

Demonstração: Seja $\left(u_{n}\right) \subset H_{0}^{1}(\Omega)$ satisfazendo $I\left(u_{n}\right) \rightarrow d$ e $I^{\prime}\left(u_{n}\right) \rightarrow 0$. Fazendo $r=2^{*}$, conforme visto no Lema 2.4, concluímos que $\left(u_{n}\right)$ é limitada. Portanto, a menos de subsequência,

$$
\begin{array}{ccc}
u_{n} \rightarrow u & \text { fracamente em } & H_{0}^{1}(\Omega), \\
u_{n} \rightarrow u & \text { fortemente em } & L^{q \sigma_{q}^{\prime}}(\Omega) .
\end{array}
$$

Denotanto $v_{n}:=u_{n}-u$, pelo Lema de Brézis-Lieb

$$
\begin{aligned}
o(1)=I^{\prime}\left(u_{n}\right) u_{n} & =\left\|u_{n}\right\|_{p}^{2}-\int b(x)\left(u_{n}^{+}\right)^{q}-\int c(x)\left(u_{n}^{+}\right)^{2^{*}} \\
& =\|u\|_{p}^{2}+\left\|v_{n}\right\|_{p}^{2}-\int b(x)\left(u^{+}\right)^{q}+o(1) \\
& -\int c(x)\left(u^{+}\right)^{2^{*}}-\int c(x)\left(v_{n}^{+}\right)^{2^{*}} \\
& =I^{\prime}(u) u+\left\|v_{n}\right\|_{p}^{2}-\int c(x)\left(v_{n}^{+}\right)^{2^{*}}+o(1)
\end{aligned}
$$

quando $n \rightarrow+\infty$. Argumentando como na prova do Lema 2.2, concluímos que $u$ é ponto crítico de $I$. Em particular $I^{\prime}(u) u=0$. Logo, para algum $l \geq 0$,

$$
\lim _{n \rightarrow \infty}\left\|v_{n}\right\|_{p}^{2}=l=\lim _{n \rightarrow \infty} \int c(x)\left(v_{n}^{+}\right)^{2^{*}}
$$


Suponhamos $l \neq 0$. Notemos que, da definição de $S$ e de $p(x)$, temos que

$$
\begin{aligned}
\left(\int c(x)\left(v_{n}^{+}\right)^{2^{*}}\right)^{2 / 2^{*}} & \leq|c|_{\infty}^{2 / 2^{*}}\left(\int\left(v_{n}^{+}\right)^{2^{*}}\right)^{2 / 2^{*}} \\
& \leq|c|_{\infty}^{2 / 2^{*}} \frac{\left\|v_{n}\right\|^{2}}{S} \\
& \leq \frac{|c|_{\infty}^{2 / 2^{*}}}{p_{0} S} \int p(x)\left|\nabla v_{n}\right|^{2}
\end{aligned}
$$

Passando ao limite a inequação acima, obtemos

$$
l \geq \frac{\left(p_{0} S\right)^{N / 2}}{|c|_{\infty}^{(N-2) / 2}}
$$

Por outro lado, é válida a seguinte igualdade

$$
d+o(1)=I\left(u_{n}\right)=I(u)+\frac{1}{2}\left\|v_{n}\right\|_{p}^{2}-\frac{1}{2^{*}} \int c(x)\left(v_{n}^{+}\right)^{2^{*}}+o(1) .
$$

Passando ao limite a equação acima e utlizando 2.4, obtemos

$$
d=I(u)+\left(\frac{1}{2}-\frac{1}{2^{*}}\right) l=I(u)+\frac{l}{N} \geq I(u)+\frac{1}{N} \frac{\left(p_{0} S\right)^{N / 2}}{|c|_{\infty}^{(N-2) / 2}} .
$$

Mas estamos supondo $u=0$ e $u=u_{0}$ os únicos possíveis pontos críticos de $I$. Como $\max \left\{I(0), I\left(u_{0}\right)\right\} \leq 0$, a desigualdade acima contradiz $d<d^{*}$, em que concluímos que $l=0$ e portanto o resultado segue.

A hipótese $\left(b c_{1}\right)$ assegura que existe $\delta>0$ tal que $B_{\delta}(a) \subset\left(\Omega_{b}^{+} \cap \Omega_{c}^{+}\right)$. Seja $\psi \in C_{0}^{\infty}(\Omega)$ tal que $\psi(x)=1$ se $x \in B_{l / 2}(a)$ e $\psi(x)=0$ se $x \in \Omega \backslash B_{l}(a)$, em que $0<l<\delta$. Para $\varepsilon>0$, definimos

$$
u_{\varepsilon}(x)=\frac{\psi(x)}{\left[\varepsilon+|x-a|^{2}\right]^{\frac{N-2}{2}}} \quad \text { e } \quad v_{\varepsilon}(x)=\frac{u_{\varepsilon}(x)}{\left|u_{\varepsilon}\right|_{2^{*}}} .
$$

Nessas condições, vale a seguinte proposição:

Proposição 2.1. Se b e c satisfazem $\left(b_{1}\right),\left(c_{1}\right)$ e $\left(b c_{1}\right)$ e $N<(2 k+2)$, então, para $\varepsilon>0$ suficientemente pequeno, a função $v_{\varepsilon}$ satisfaz

$$
\max _{t>0} I\left(u_{0}+t v_{\varepsilon}\right)<d^{*}=I\left(u_{0}\right)+\frac{1}{N} \frac{\left(p_{0} S\right)^{N / 2}}{|c|_{\infty}^{(N-2) / 2}} .
$$


Demonstração: Para todo $\varepsilon>0$, a função $t \rightarrow I\left(u_{0}+t v_{\varepsilon}\right)$ atinge seu valor máximo em $t_{\varepsilon}>0$ e, por um argumento análogo ao utilizado no Lema 1.4, temos que $t_{\varepsilon}=O(1)$ quando $\varepsilon \rightarrow 0^{+}$.

Vamos usar agora a definição de $I$ e o fato de que $I^{\prime}\left(u_{0}\right) v_{\varepsilon}=0$ para calcular

$$
\begin{aligned}
m_{\varepsilon}: & =I\left(u_{0}+t_{\varepsilon} v_{\varepsilon}\right)=\frac{1}{2} \int p(x)\left|\nabla\left(u_{0}+t_{\varepsilon} v_{\varepsilon}\right)\right|^{2}-\frac{1}{q} \int b(x)\left(u_{0}+t_{\varepsilon} v_{\varepsilon}\right)^{q} \\
& -\frac{1}{2^{*}} \int c(x)\left(u_{0}+t_{\varepsilon} v_{\varepsilon}\right)^{2^{*}} \\
& =\frac{1}{2} \int p(x)\left|\nabla u_{0}\right|^{2}+t_{\varepsilon} \int_{B_{l}(a)} p(x)\left(\nabla u_{0} \cdot \nabla v_{\varepsilon}\right) d x+\frac{t_{\varepsilon}^{2}}{2} \int_{B_{l}(a)} p(x)\left|\nabla v_{\varepsilon}\right|^{2} d x \\
& -\frac{1}{q} \int b(x)\left(u_{0}+t_{\varepsilon} v_{\varepsilon}\right)^{q}-\frac{1}{2^{*}} \int c(x)\left(u_{0}+t_{\varepsilon} v_{\varepsilon}\right)^{2^{*}} \\
& =\frac{1}{2} \int p(x)\left|\nabla u_{0}\right|^{2}-\frac{1}{q} \int b(x)\left(u_{0}^{+}\right)^{q}-\frac{1}{r} \int c(x)\left(u_{0}^{+}\right)^{r}+t_{\varepsilon} \int_{B_{l}(a)} p(x)\left(\nabla u_{0} \cdot \nabla v_{\varepsilon}\right) d x \\
& -t_{\varepsilon}\left(\int_{B_{l}(a)} b(x) u_{0}^{q-1} v_{\varepsilon} d x+\int_{B_{l}(a)} c(x) u_{0}^{2^{*}-1} v_{\varepsilon} d x\right)+\frac{t_{\varepsilon}^{2}}{2}\left\|v_{\varepsilon}\right\|_{p}^{2}-\frac{1}{q} A_{\varepsilon}-\frac{1}{2^{*}} D_{\varepsilon} \\
& =I\left(u_{0}\right)+t_{\varepsilon} I^{\prime}\left(u_{0}\right) v_{\varepsilon}+\frac{t_{\varepsilon}^{2}}{2}\left\|v_{\varepsilon}\right\|_{p}^{2}-\frac{1}{q} A_{\varepsilon}-\frac{1}{2^{*}} D_{\varepsilon}
\end{aligned}
$$

portanto

$$
\max _{t>0} I\left(u_{0}+t v_{\varepsilon}\right)=m_{\varepsilon}=I\left(u_{0}\right)+\frac{t_{\varepsilon}^{2}}{2}\left\|v_{\varepsilon}\right\|_{p}^{2}-\frac{1}{q} A_{\varepsilon}-\frac{1}{2^{*}} D_{\varepsilon}
$$

em que

$$
A_{\varepsilon}:=\int b(x)\left[\left(u_{0}+t_{\varepsilon} v_{\varepsilon}\right)^{q}-u_{0}^{q}-q t_{\varepsilon} u_{0}^{q-1} v_{\varepsilon}\right]
$$

e

$$
D_{\varepsilon}:=\int c(x)\left[\left(u_{0}+t_{\varepsilon} v_{\varepsilon}\right)^{2^{*}}-u_{0}^{2^{*}}-2^{*} t_{\varepsilon} u_{0}^{2^{*}-1} v_{\varepsilon}\right]
$$

Observem que, como $v_{\varepsilon} \equiv 0$ fora de $B_{l}(a)$, então

$$
\begin{aligned}
A_{\varepsilon} & =\int_{B_{l}(a)} b(x)\left[\left(u_{0}+t_{\varepsilon} v_{\varepsilon}\right)^{q}-u_{0}^{q}-q t_{\varepsilon} u_{0}^{q-1} v_{\varepsilon}\right] d x+\int_{\Omega \backslash B_{l}(a)} b(x)\left[\left(u_{0}+0\right)^{q}-u_{0}^{q}\right] d x \\
& =\int_{B_{l}(a)} b(x)\left[\left(u_{0}+t_{\varepsilon} v_{\varepsilon}\right)^{q}-u_{0}^{q}-q t_{\varepsilon} u_{0}^{q-1} v_{\varepsilon}\right] d x
\end{aligned}
$$

Analogamente

$$
D_{\varepsilon}=\int_{B_{l}(a)} c(x)\left[\left(u_{0}+t_{\varepsilon} v_{\varepsilon}\right)^{2^{*}}-u_{0}^{2^{*}}-2^{*} t_{\varepsilon} u_{0}^{2^{*}-1} v_{\varepsilon}\right] d x
$$


Como $u_{0} \geq 0$ em $B_{l}(a)$, podemos usar o Teorema do Valor Médio para obter $\eta(x) \in$ $[0,1]$ mensurável tal que

$$
\begin{aligned}
\left(u_{0}(x)+t_{\varepsilon} v_{\varepsilon}(x)\right)^{q}-u_{0}(x)^{q} & =q\left(u_{0}(x)+\eta(x) t_{\varepsilon} v_{\varepsilon}(x)\right)^{q-1} t_{\varepsilon} v_{\varepsilon} \\
& \geq q t_{\varepsilon} u_{0}(x)^{q-1} v_{\varepsilon}(x)
\end{aligned}
$$

qualquer que seja $x \in B_{l}(a)$. Logo, como $b(x) \geq 0$ em $B_{l}(a)$, segue que $A_{\varepsilon} \geq 0$.

Para estimar $D_{\varepsilon}$, vamos usar a seguinte desigualdade que se encontra em [7]. Para $m, n \geq 0, s>2$ e $1<\mu<s-1$,

$$
(m+n)^{s} \geq m^{s}+n^{s}+s m^{s-1} n+s m n^{s-1}-C_{\mu} n^{\mu} m^{s-\mu}
$$

em que $C_{\mu}$ é uma constante positiva. Escolhendo $m=u_{0}, n=t_{\varepsilon} v_{\varepsilon}$ e $s=2^{*}$ teremos que

$$
\left(u_{0}+t_{\varepsilon} v_{\varepsilon}\right)^{2^{*}}-u_{0}^{2^{*}}-2^{*} u_{0}^{2^{*}-1} t_{\varepsilon} v_{\varepsilon} \geq t_{\varepsilon}^{2^{*}} v_{\varepsilon}^{2^{*}}+2^{*} t_{\varepsilon}^{2^{*}-1} u_{0} v_{\varepsilon}^{2^{*}-1}-C_{\mu} t_{\varepsilon}^{\mu} v_{\varepsilon}^{\mu} u_{0}^{2^{*}-\mu}
$$

Da maneira que foi definido $D_{\varepsilon}$, da desigualdade acima e lembrando que $c(x) \geq 0$ em $B_{l}(a)$, temos que

$$
D_{\varepsilon} \geq \int_{B_{l}(a)} c(x)\left[t_{\varepsilon}^{2^{*}} v_{\varepsilon}^{2^{*}}+2^{*} t_{\varepsilon}^{2^{*}-1} u_{0} v_{\varepsilon}^{2^{*}-1}-C_{\mu} t_{\varepsilon}^{\mu} v_{\varepsilon}^{\mu} u_{0}^{2^{*}-\mu}\right] d x
$$

Substituindo as inequações acima em (2.5), obtemos

$$
\begin{aligned}
m_{\varepsilon} & \leq I\left(u_{0}\right)+\left(\frac{t_{\varepsilon}^{2}}{2}\left\|v_{\varepsilon}\right\|_{p}^{2}-\frac{t_{\varepsilon}^{2^{*}}}{2^{*}}|c|_{\infty}\right)+\frac{t_{\varepsilon}^{2^{*}}}{2^{*}} \int_{B_{l}(a)}\left(|c|_{\infty}-c(x)\right) v_{\varepsilon}^{2^{*}} d x \\
& -t_{\varepsilon}^{2^{*}-1} \int_{B_{l}(a)} c(x) u_{0} v_{\varepsilon}^{2^{*}-1} d x+C_{\mu} \frac{t_{\varepsilon}^{\mu}}{2^{*}} \int_{B_{l}(a)} c(x) u_{0}^{2^{*}-\mu} v_{\varepsilon}^{\mu} d x
\end{aligned}
$$

em que usamos o fato de que $\int_{B_{l}(a)} v_{\varepsilon}^{2^{*}} d x=1$. Faremos algumas estimativas para os termos do lado direito da desigualdade acima.

Se $B$ e $C$ são constantes positivas, então, a função $f:[0,+\infty) \mapsto \mathbb{R}$ definida por

$$
f(t)=\frac{t^{2}}{2} B-\frac{t^{2^{*}}}{2^{*}} C,
$$

atinge seu máximo no ponto $t_{0}=(B / C)^{1 /\left(2^{*}-2\right)} \mathrm{com}$

$$
f\left(t_{0}\right)=\frac{1}{N} \frac{B^{N / 2}}{C^{(N-2) / 2}}
$$


Portanto, fazendo $B=\left\|v_{\varepsilon}\right\|_{p}^{2}$ e $C=|c|_{\infty}$ concluímos que

$$
\max _{t \geq 0}\left(\frac{t^{2}}{2}\left\|v_{\varepsilon}\right\|_{p}^{2}-\frac{t^{2^{*}}}{2^{*}}|c|_{\infty}\right)=\frac{1}{N} \frac{\left\|v_{\varepsilon}\right\|_{p}^{N}}{|c|_{\infty}^{(N-2) / 2}}
$$

Para todo $\sigma>N /(N-2)$, podemos proceder como em (1.23) e calcular

$$
\int\left|u_{\varepsilon}\right|^{\sigma}=\varepsilon^{\frac{N}{2}-\sigma \frac{(N-2)}{2}} \int_{B(l / \sqrt{\varepsilon}, 0)} \frac{1}{\left(1+|y|^{2}\right)^{\sigma \frac{(N-2)}{2}}} d y+O(1) .
$$

Como

$$
\int_{B(l / \sqrt{\varepsilon}, 0)} \frac{1}{\left(1+|y|^{2}\right)^{\sigma \frac{(N-2)}{2}}} d y \leq \int_{B_{1}(0)} \frac{1}{\left(1+|y|^{2}\right)^{\sigma \frac{(N-2)}{2}}} d y+\int_{B_{1}(0)^{C}}|y|^{-\sigma(N-2)} d y
$$

e $\sigma>N /(N-2)$, a última integral é finita, de modo que

$$
\int\left|u_{\varepsilon}\right|^{\sigma}=O\left(\varepsilon^{\frac{N}{2}-\frac{\sigma(N-2)}{2}}\right)+O(1), \quad \text { para todo } \quad \sigma>\frac{N}{N-2} .
$$

Por outro lado, uma estimativa devida a Brézis-Nirenberg (cf. [9, pg 444]) fornece

$$
\left|u_{\varepsilon}\right|_{2^{*}}^{2}=K_{2} \varepsilon^{-\frac{N-2}{2}}+O(\varepsilon)
$$

em que $K_{2}=K_{2}(N)>0$. Assim, pelo Teorema do Valor Médio, existe $\delta \in(0,1)$ tal que

$$
\begin{aligned}
\left|u_{\varepsilon}\right|_{2^{*}}^{\sigma} & =\left(\left|u_{\varepsilon}\right|_{2^{*}}^{2}\right)^{\frac{\sigma}{2}} \\
& =\left(K_{2} \varepsilon^{-\frac{(N-2)}{2}}+O(\varepsilon)\right)^{\frac{\sigma}{2}} \\
& =K_{2}^{\sigma / 2} \varepsilon^{-\frac{\sigma(N-2)}{4}}+\frac{\sigma}{2}\left(K_{2} \varepsilon^{-\frac{(N-2)}{2}}+\delta O(\varepsilon)\right)^{\frac{\sigma}{2}-1} O(\varepsilon),
\end{aligned}
$$

$\log O$

$$
\left|u_{\varepsilon}\right|_{2^{*}}^{\sigma}=O\left(\varepsilon^{-\frac{\sigma(N-2)}{4}}\right)+O(1)
$$

Consequentemente, das estimativas acima, obtemos

$$
\begin{aligned}
\int\left|v_{\varepsilon}\right|^{\sigma} & =\frac{1}{\left|u_{\varepsilon}\right|_{2^{*}}^{\sigma}} \int\left|u_{\varepsilon}\right|^{\sigma} \\
& =\frac{O\left(\varepsilon^{\frac{N}{2}-\frac{\sigma(N-2)}{2}}\right)+O(1)}{O\left(\varepsilon^{-\frac{\sigma(N-2)}{4}}\right)+O(1)} \\
& =O\left(\varepsilon^{\frac{N}{2}-\frac{\sigma(N-2)}{4}}\right)+O\left(\varepsilon^{\frac{\sigma(N-2)}{4}}\right) .
\end{aligned}
$$


Como estamos supondo $\sigma>N /(N-2)$, podemos concluir que

$$
\frac{N}{2}-\frac{\sigma(N-2)}{4}<\frac{\sigma(N-2)}{4}
$$

Portanto, a estimativa acima fica

$$
\int\left|v_{\varepsilon}\right|^{\sigma}=O\left(\varepsilon^{\frac{N}{2}-\frac{\sigma(N-2)}{2}}\right)=O\left(\varepsilon^{(N(2-\sigma)+2 \sigma) / 4}\right)
$$

Como a desigualdade em (2.6) foi usada com $\mu \in\left(1,2^{*}-1\right)$, vamos escolher $\mu=\frac{N+1}{N-2}$ e usar a igualdade acima com $\sigma=\mu$ para obter

$$
C_{\mu} \frac{t_{\varepsilon}^{\mu}}{2^{*}} \int c(x) u_{0}^{2^{*}-\mu} v_{\varepsilon}^{\mu}=O\left(\varepsilon^{(N-1) / 4}\right)
$$

pois $t_{\varepsilon}$ é limitado e $b(x)$ e $u_{0}$ são limitados em $B_{l}(a)$. O mesmo argumento nos permite obter $A_{0}>0$ tal que

$$
t_{\varepsilon}^{2^{*}-1} \int c(x) u_{0} v_{\varepsilon}^{2^{*}-1}=A_{0} O\left(\varepsilon^{(N-2) / 4}\right) .
$$

Agora, podemos usar a identidade obtida em (2.9) com $\sigma=2^{*}$ para obter

$$
\left|u_{\varepsilon}\right|_{2^{*}}^{2^{*}}=O\left(\varepsilon^{-N / 2}\right)+O(1)
$$

Por outro lado, utilizando a hipótese $\left(b c_{1}\right)$ podemos computar a seguinte integral

$$
\begin{aligned}
\int_{B_{l}(a)}\left(|c|_{\infty}-c(x)\right) v_{\varepsilon}^{2^{*}} d x & =\frac{1}{\left|u_{\varepsilon}\right|_{2^{*}}^{2^{*}}} \int_{B_{l}(a)}\left(|c|_{\infty}-c(x)\right) u_{\varepsilon}^{2^{*}} d x \\
& \leq \frac{M}{O\left(\varepsilon^{-N / 2}\right)+O(1)} \int_{B_{l}(a)} \frac{|\psi(x)|^{2^{*}}|x-a|^{\gamma}}{\left[\varepsilon+|x-a|^{2}\right]^{N}} d x \\
& \leq O\left(\varepsilon^{N / 2}\right) \int_{B_{l}(a)} \frac{|x-a|^{\gamma}}{\left[\varepsilon+|x-a|^{2}\right]^{N}} d x
\end{aligned}
$$

Fazendo $x-a=\sqrt{\varepsilon} y$ e utilizando o Teorema da Mudança de Variável, temos que

$$
\int_{B(l, a)} \frac{|x-a|^{\gamma}}{\left[\varepsilon+|x-a|^{2}\right]^{N}} d x=\int_{B(l / \sqrt{\varepsilon}, 0)} \frac{\varepsilon^{\frac{\gamma}{2}} \varepsilon^{\frac{N}{2}} \varepsilon^{-N}|y|^{\gamma}}{\left[1+|y|^{2}\right]^{N}} d y .
$$


Mas, por Fubini,

$$
\begin{aligned}
\int_{B(l / \sqrt{\varepsilon}, 0)} \frac{|y|^{\gamma}}{\left[1+|y|^{2}\right]^{N}} d y & \leq \omega_{N}\left(\int_{0}^{1} \frac{r^{\gamma} r^{N-1}}{\left(1+r^{2}\right)^{N}} d r+\int_{1}^{l \sqrt{\varepsilon}} r^{\gamma-2 N+N-1} d r\right) \\
& =O(1)+O\left(\varepsilon^{-\frac{\gamma}{2}+\frac{N}{2}}\right),
\end{aligned}
$$

em que $\omega_{N}$ é a área da esfera unitária em $\mathbb{R}^{N}$. Portanto, por (2.13) e da estimativa acima, concluímos que

$$
\int_{B_{l}(a)}\left(|c|_{\infty}-c(x)\right) v_{\varepsilon}^{2^{*}} d x=O\left(\varepsilon^{\frac{\gamma}{2}}\right)+O\left(\varepsilon^{\frac{N}{2}}\right) .
$$

Portanto, das estimativas obtidas em (2.7), (2.8), (2.10), (2.11) e (2.14) conluímos que

$$
m_{\varepsilon} \leq I\left(u_{0}\right)+\frac{1}{N} \frac{\left\|v_{\varepsilon}\right\|_{p}^{N}}{|c|_{\infty}^{(N-2) / 2}}+O\left(\varepsilon^{(N-1) / 4}\right)+O\left(\varepsilon^{\gamma / 2}\right)+O\left(\varepsilon^{N / 2}\right)-A_{0} O\left(\varepsilon^{(N-2) / 4}\right) .
$$

Mas podemos supor, sem perda de generalidade $\gamma / 2<(N-1) / 4$. De fato, se escrevermos $\gamma=\gamma_{1}+\gamma_{2}$ tal que $\gamma_{1}<(N-1) / 2$, como $\Omega$ é limitado, existe $M_{1}>0$ tal que $|x-a|^{\gamma_{2}} \leq M_{2}$. Dessa forma, da hipótese $\left(b c_{1}\right)$,

$$
|c|_{\infty}-c(x) \leq M|x-a|^{\gamma} \leq M|x-a|^{\gamma_{1}}|x-a|^{\gamma_{2}} \leq M M_{1}|x-a|^{\gamma_{1}}=\widetilde{M}|x-a|^{\gamma_{1}} .
$$

Trocando, se necessário, $\gamma$ por $\gamma_{1}$, teremos $\gamma / 2<(N-1) / 4$. Além disso, como $(N-1) / 4<$ $N / 2$, a inequação acima fica

$$
m_{\varepsilon} \leq I\left(u_{0}\right)+\frac{1}{N} \frac{\left\|v_{\varepsilon}\right\|_{p}^{N}}{|c|_{\infty}^{(N-2) / 2}}+O\left(\varepsilon^{\gamma / 2}\right)-A_{0} O\left(\varepsilon^{(N-2) / 4}\right)
$$

A prova agora será dividida em vários passos. Observamos inicialmente que, usando as estimativas obtidas em (1.36), (1.37), (1.40) e (1.35), obtemos

$$
\left\|v_{\varepsilon}\right\|_{p}^{2} \leq \begin{cases}p_{0} S+O(\varepsilon), & N=4 \text { e } k>2 ; \\ p_{0} S+O\left(\varepsilon^{\frac{N-2}{2}}\right), & N \geq 5 \text { e } N-2<k ; \\ p_{0} S+O\left(\varepsilon^{\frac{N-2}{2}}|\log \varepsilon|\right), & N \geq 5 \text { e } N-2=k ; \\ p_{0} S+O\left(\varepsilon^{\frac{k}{2}}\right), & N \geq 5 \text { e } N-2>k .\end{cases}
$$

Caso 1: Quando $N=4$, usamos o Teorema do Valor Médio para obter 


$$
\left(\left\|v_{\varepsilon}\right\|_{p}^{2}\right)^{N / 2}=\left(p_{0} S\right)^{N / 2}+O(\varepsilon) .
$$

Dessa forma a expressão em (2.15) fica

$$
\begin{aligned}
m_{\varepsilon} & \leq I\left(u_{0}\right)+\frac{1}{N} \frac{\left(p_{0} S\right)^{N / 2}}{|b|_{\infty}^{(N-2) / 2}}+O(\varepsilon)+O\left(\varepsilon^{\gamma / 2}\right)-A_{0} O\left(\varepsilon^{1 / 2}\right) \\
& =d^{*}+O\left(\varepsilon^{1 / 2}\right)\left(O\left(\varepsilon^{1 / 2}\right)+O\left(\varepsilon^{(\gamma-1) / 2}\right)-A_{0}\right)
\end{aligned}
$$

quando $\varepsilon \rightarrow 0^{+}$. Como $\gamma>(N-2) / 2=1$, então $m_{\varepsilon}<d^{*}$ para $\varepsilon>0$ suficientemente pequeno.

Caso 2: Se $N \geq 5$ e $N<k+2$, usando novamente o Teorema do Valor Médio obtemos

$$
\left(\left\|v_{\varepsilon}\right\|_{p}^{2}\right)^{N / 2}=\left(p_{0} S\right)^{N / 2}+O\left(\varepsilon^{(N-2) / 2}\right)
$$

Portanto, por (2.15) teremos

$$
\begin{aligned}
m_{\varepsilon} & \leq I\left(u_{0}\right)+\frac{1}{N} \frac{\left(p_{0} S\right)^{N / 2}}{|c|_{\infty}^{(N-2) / 2}}+O\left(\varepsilon^{(N-2) / 2}\right)+O\left(\varepsilon^{\gamma / 2}\right)-A_{0} O\left(\varepsilon^{(N-2) / 4}\right) \\
& =d^{*}+O\left(\varepsilon^{(N-2) / 4}\right)\left(O\left(\varepsilon^{(N-2) / 4}\right)+O\left(\varepsilon^{\frac{\gamma}{2}-\frac{(N-2)}{4}}\right)-A_{0}\right)
\end{aligned}
$$

e como $\gamma / 2>(N-2) / 4$, mais uma vez concluímos que $m_{\varepsilon}<d^{*}$ quando $\varepsilon \rightarrow 0^{+}$.

Caso 3: Se $N \geq 5$ e $N=k+2$, analogamente aos casos anteriores, teremos por (2.15) que

$$
\begin{aligned}
m_{\varepsilon} & \leq I\left(u_{0}\right)+\frac{1}{N} \frac{\left(p_{0} S\right)^{N / 2}}{|c|_{\infty}^{(N-2) / 2}}+O\left(\varepsilon^{\frac{N-2}{2}}|\log \varepsilon|\right)+O\left(\varepsilon^{\gamma / 2}\right)-A_{0} O\left(\varepsilon^{(N-2) / 4}\right) \\
& =d^{*}+O\left(\varepsilon^{(N-2) / 4}\right)\left(O\left(\varepsilon^{\frac{N-2}{4}}|\log \varepsilon|\right)+O\left(\varepsilon^{\frac{\gamma}{2}-\frac{(N-2)}{4}}\right)-A_{0}\right) .
\end{aligned}
$$

Como $\lim _{\varepsilon \rightarrow 0^{+}} \varepsilon^{\frac{N-2}{4}}|\log \varepsilon|=0$, concluímos que $m_{\varepsilon}<d^{*}$ quando $\varepsilon \rightarrow 0^{+}$.

Caso 4: Para o caso em que $N \geq 5$ e $N>k+2$, argumentando como antes

$$
\left(\left\|v_{\varepsilon}\right\|_{p}^{2}\right)^{N / 2}=\left(p_{0} S\right)^{N / 2}+O\left(\varepsilon^{k / 2}\right) .
$$


Lembrando que estamos supondo adicionalmente $k>(N-2) / 2$, teremos

$$
\begin{aligned}
m_{\varepsilon} & \leq I\left(u_{0}\right)+\frac{1}{N} \frac{\left(p_{0} S\right)^{N / 2}}{|c|_{\infty}^{(N-2) / 2}}+O\left(\varepsilon^{k / 2}\right)+O\left(\varepsilon^{\gamma / 2}\right)-A_{0} O\left(\varepsilon^{(N-2) / 4}\right) \\
& =d^{*}+O\left(\varepsilon^{(N-2) / 4}\right)\left(O\left(\varepsilon^{\frac{k}{2}-\frac{(N-2)}{4}}\right)+O\left(\varepsilon^{\frac{\gamma}{2}-\frac{(N-2)}{4}}\right)-A_{0}\right)
\end{aligned}
$$

e uma vez que $2 k-N+2>0$, o resultado segue, e assim, encerra-se a prova da proposição.

Estamos, então, em condições de demonstrar o segundo teorema do capítulo.

Demonstração do Teorema 2.2: A prova da existência de uma solução com energia negativa é a mesma do Teorema 2.1. Suponhamos, por contradição, que não exista outra solução além de zero e de $u_{0}$. Por um lado, sabemos pelo Lema 2.3 que $I\left(u_{0}+t v_{\varepsilon}\right) \rightarrow-\infty$ quando $t \rightarrow+\infty$. Tomemos $e:=u_{0}+t v_{\varepsilon}$ tal que, para algum $t>0$ suficientemente grande, $I(e) \leq I\left(u_{0}\right)$. Seja

$$
\bar{d}=\inf _{\gamma \in \Gamma} \max _{t \in[0,1]} I(\gamma(t))
$$

em que

$$
\Gamma:=\left\{\gamma \in C\left([0,1], H_{0}^{1}(\Omega)\right): \gamma(0)=u_{0}, \gamma(1)=e\right\} .
$$

Logo, pelo Teorema do Passo da Montanha e a Proposição 2.1, existe uma sequência $\left(u_{n}\right) \subset H_{0}^{1}(\Omega)$ satisfazendo

$$
I\left(u_{n}\right) \rightarrow \bar{d}<d^{*}=I\left(u_{0}\right)+\frac{1}{N} \frac{\left(p_{0} S\right)^{N / 2}}{|c|_{\infty}^{(N-2) / 2}}
$$

e $I^{\prime}\left(u_{n}\right) \rightarrow 0$. Mas pelo Lema 2.5, como estamos supondo que $u_{0}$ e zero únicos pontos críticos de $I$, então $\left(u_{n}\right)$ satisfaz $(P S)_{\bar{d}}$ sempre que $\bar{d}<d^{*}$. Ou seja, a menos de subsequência, $u_{n} \rightarrow u_{1}$ em $H_{0}^{1}(\Omega)$ e além disso $u_{1}$ é tal que $I\left(u_{1}\right)=\bar{d} \geq 0$ e $I^{\prime}\left(u_{1}\right)=0$. Mas pela definição de $\bar{d}$ e pelo Lema 2.1, concluímos que $I\left(u_{1}\right)=\bar{d} \geq \alpha>0$, mostrando que nesse caso a solução não pode ser $u_{0}$, que tem energia negativa, nem zero, contrariando o fato de termos suposto não haver solução além de $u_{0}$ e zero, um absurdo. Logo, existe uma terceira solução diferente de $u_{0}$ e zero. 


\section{CAPÍTULO 3}

\section{Sistema com perturbação superlinear}

Este capítulo será dedicado ao estudo da existência de soluções fracas para o sistema

$(P Q) \quad\left\{\begin{aligned}-\operatorname{div}(p(x) \nabla u) & =b u+c v+\frac{\alpha}{2^{*}} u|u|^{\alpha-2}|v|^{\beta}, & & x \in \Omega, \\ -\operatorname{div}(q(x) \nabla v) & =c u+d v+\frac{\beta}{2^{*}}|u|^{\alpha} v|v|^{\beta-2}, & & x \in \Omega, \\ u, v & =0, & & x \in \partial \Omega,\end{aligned}\right.$

em que $\Omega \subset \mathbb{R}^{N}, N \geq 4$, é um domínio limitado com fronteira suave, $b, c, d \in \mathbb{R}$ e $\alpha, \beta>1$ são tais que $\alpha+\beta=2^{*}$. A função $p$ satisfaz as seguintes condições

$\left(p_{1}\right) p \in H^{1}(\Omega) \cap C(\Omega) ;$

$\left(p_{2}\right)$ existe um ponto $a \in \Omega$ tal que

$$
p(a)=p_{0}:=\min \{p(x): x \in \bar{\Omega}\}>0
$$

$\left(p_{3}\right)$ existe $k>0, \beta_{k}>0$ e $\theta_{p}$ tais que, numa vizinhança de $a$, a função $p$ é da forma

$$
p(x)=p_{0}+\beta_{k}|x-a|^{k}+\theta_{p}(x)|x-a|^{k},
$$

$$
\text { com } \lim _{x \rightarrow a} \theta_{p}(x)=0 .
$$

A função $q$ satisfaz: 
$\left(q_{1}\right) q \in H^{1}(\Omega) \cap C(\Omega) ;$

$\left(q_{2}\right) q(a)=q_{0}:=\min \{q(x): x \in \bar{\Omega}\}>0 ;$

$\left(q_{3}\right)$ existe $j>0, \gamma_{j}>0$ e $\theta_{q}$ tais que, numa vizinhança de $a$, a função $q$ é da forma

$$
q(x)=q_{0}+\gamma_{j}|x-a|^{j}+\theta_{q}(x)|x-a|^{j},
$$

$\operatorname{com} \lim _{x \rightarrow a} \theta_{q}(x)=0$.

Os resultados estão relacionados com a parte linear do problema, dada pela matriz real

$$
A=\left(\begin{array}{ll}
b & c \\
c & d
\end{array}\right) .
$$

Se denotarmos por $\mu_{1} \leq \mu_{2}$ os seus auto-valores reais, temos que

$$
\mu_{1}|Z|_{\mathbb{R}^{2}}^{2} \leq\langle A Z, Z\rangle_{\mathbb{R}^{2}} \leq \mu_{2}|Z|_{\mathbb{R}^{2}}^{2}, \quad \forall Z \in \mathbb{R}^{2}
$$

Antes de enunciar nosso resultado de não existência, vale lembrar que um domínio $\Omega$ é dito estrelado com relação ao ponto $a$ se toda semi-reta partindo de $a$ intercepta a fronteira de $\Omega$ em somente um ponto. Nesse caso, temos que $\eta(x) \cdot(x-a)>0$ para todo $x \in \partial \Omega$.

Teorema 3.1. Suponha que $\Omega$ é um domínio estrelado com respeito ao ponto a $\in \Omega$ e que $p, q \in C^{1}(\bar{\Omega})$ satisfazem

$$
\nabla p(x) \cdot(x-a) \geq 0, \quad \nabla q(x) \cdot(x-a) \geq 0,
$$

para todo $x \in \Omega$. Se $\mu_{2} \leq 0$, então o problema $(P Q)$ não possui solução positiva.

Para enunciar o nosso resultado de existência, vamos introduzir os números

$$
\lambda_{1, p}:=\inf \left\{\int p(x)|\nabla u|^{2}: u \in H_{0}^{1}(\Omega), \int u^{2}=1\right\}>0
$$

$$
\lambda_{1, q}:=\inf \left\{\int q(x)|\nabla u|^{2}: u \in H_{0}^{1}(\Omega), \int u^{2}=1\right\}>0 .
$$


Precisamos, ainda, definir a seguinte quantidade

$$
\vartheta(N, k, j):= \begin{cases}0, & \text { se } \min \{k, j\}>2, \\ \vartheta_{1} \beta_{2}, & \text { se } k=2, j>2, \\ \vartheta_{1} \gamma_{2}, & \text { se } k>2, j=2, \\ \vartheta_{1} \max \left\{\beta_{2}, \gamma_{2}\right\}, & \text { se } k=2, j=2,\end{cases}
$$

em que

$$
\vartheta_{1}:=\left\{\begin{array}{l}
4, \text { se } N=4, \\
(N-2)^{2}\left(\int_{\mathbb{R}^{N}} \frac{|y|^{4}}{\left(1+|y|^{2}\right)^{N}} d y\right)\left(\int_{\mathbb{R}^{N}} \frac{1}{\left(1+|y|^{2}\right)^{N-2}} d y\right)^{-1}, \text { se } N \geq 5 .
\end{array}\right.
$$

Podemos agora estabelecer o nosso resultado de existência como se segue.

Teorema 3.2. Se as funções p e q satisfazem $\left(p_{1}\right)-\left(p_{3}\right)$ e $\left(q_{1}\right)-\left(q_{3}\right)$, a matriz A é tal que $c>0$ e

$$
\vartheta(N, k, j)<\mu_{1} \leq \mu_{2}<\min \left\{\lambda_{1, p}, \lambda_{1, q}\right\}
$$

então o problema $(P Q)$ tem pelo menos uma solução positiva.

\subsection{Resultado de não existência}

Nessa seção provamos o Teorema 3.1. O ponto chave é o seguinte lema, que é uma adaptação da desigualdade de Pohozaev [28]:

Lema 3.1. Se $p, q \in C^{1}(\Omega), F \in C^{1}\left(\mathbb{R}^{2}, \mathbb{R}\right)$ é tal que $F(0,0)=0$ e $u, v \in C^{2}(\Omega) \cap C^{1}(\bar{\Omega})$ são soluções do sistema

$$
\left\{\begin{aligned}
-\operatorname{div}(p(x) \nabla u) & =F_{u}(u, v), & & x \in \Omega \\
-\operatorname{div}(q(x) \nabla u) & =F_{v}(u, v), & & x \in \Omega \\
u, v & =0, & & x \in \partial \Omega
\end{aligned}\right.
$$

então vale a seguinte identidade

$$
\int\left(p(x)|\nabla u|^{2}+q(x)|\nabla v|^{2}\right)+\frac{1}{N-2}\left[\int G(x)+\int_{\partial \Omega} H(x) d S\right]=2^{*} \int F(u, v),
$$

em que

$$
G(x)=\nabla p(x) \cdot(x-a)|\nabla u|^{2}+\nabla q(x) \cdot(x-a)|\nabla v|^{2},
$$




$$
H(x)=\left[\left(p(x)(\nabla u \cdot \eta)^{2}+q(x)(\nabla v \cdot \eta)^{2}\right](x-a) \cdot \eta\right.
$$

e $\eta$ é o vetor normal unitário exterior à $\partial \Omega$.

Demonstração: Estabeleceremos inicialmente a seguinte notação

$$
u_{i}=\frac{\partial u}{\partial x_{i}}, \quad x=\left(x_{1}, \cdots, x_{N}\right) \in \mathbb{R}^{N}
$$

Observe que, usando o Teorema de Schwarz, obtemos

$$
\begin{aligned}
u_{i} \frac{\partial}{\partial x_{i}}((x-a) \cdot \nabla u) & =\left(u_{i}\right)^{2}+\left(x_{1}-a_{1}\right) u_{i}\left(u_{1}\right)_{i}+\cdots+\left(x_{N}-a_{N}\right) u_{i}\left(u_{N}\right)_{i} \\
& =\left(u_{i}\right)^{2}+\left(x_{1}-a_{1}\right) \frac{\partial}{\partial x_{1}}\left[\frac{\left(u_{i}\right)^{2}}{2}\right]+\cdots+\left(x_{N}-a_{N}\right) \frac{\partial}{\partial x_{N}}\left[\frac{\left(u_{i}\right)^{2}}{2}\right]
\end{aligned}
$$

Somando para $i$ variando de 1 até $N$ obtemos

$$
\begin{aligned}
\sum_{i=1}^{N}\left(u_{i} \frac{\partial}{\partial x_{i}}((x-a) \cdot \nabla u)\right) & =\sum_{i=1}^{N}\left(u_{i}\right)^{2}+\left(x_{1}-a_{1}\right) \frac{\partial}{\partial x_{1}}\left[\sum_{i=1}^{N} \frac{\left(u_{i}\right)^{2}}{2}\right] \\
& +\cdots+\left(x_{N}-a_{N}\right) \frac{\partial}{\partial x_{N}}\left[\sum_{i=1}^{N} \frac{\left(u_{i}\right)^{2}}{2}\right] \\
& =|\nabla u|^{2}+(x-a) \cdot \nabla\left[\frac{|\nabla u|^{2}}{2}\right] .
\end{aligned}
$$

Além disso, temos também que

$$
\begin{aligned}
\operatorname{div}\left((x-a) \frac{|\nabla u|^{2}}{2}\right) & =\sum_{i=1}^{N} \frac{\partial}{\partial x_{i}}\left[\left(x_{i}-a_{i}\right) \frac{|\nabla u|^{2}}{2}\right] \\
& =\sum_{i=1}^{N}\left[\frac{|\nabla u|^{2}}{2}+\left(x_{i}-a_{i}\right) \frac{\partial}{\partial x_{i}}\left[\frac{|\nabla u|^{2}}{2}\right]\right] \\
& =\frac{N}{2}|\nabla u|^{2}+(x-a) \cdot \nabla\left[\frac{|\nabla u|^{2}}{2}\right]
\end{aligned}
$$


Utilizando as identidades acima, podemos calcular o seguinte divergente

$$
\begin{aligned}
\operatorname{div}(p(x) \nabla u((x-a) \cdot \nabla u)) & =\operatorname{div}(p(x) \nabla u)((x-a) \cdot \nabla u)+p(x) \sum_{i=1}^{N} u_{i} \frac{\partial}{\partial x_{i}}((x-a) \cdot \nabla u) \\
& =\operatorname{div}(p(x) \nabla u)((x-a) \cdot \nabla u) \\
& +p(x)\left(|\nabla u|^{2}+(x-a) \cdot \nabla\left[\frac{|\nabla u|^{2}}{2}\right]\right) \\
& =\operatorname{div}(p(x) \nabla u)((x-a) \cdot \nabla u) \\
& +p(x)\left(\frac{N}{2}|\nabla u|^{2}+(x-a) \cdot \nabla\left[\frac{|\nabla u|^{2}}{2}\right]\right) \\
& +p(x)\left(1-\frac{N}{2}\right)|\nabla u|^{2} \\
& =\operatorname{div}(p(x) \nabla u)((x-a) \cdot \nabla u) \\
& +p(x) \operatorname{div}\left((x-a) \frac{|\nabla u|^{2}}{2}\right)+p(x)\left(\frac{2-N}{2}\right)|\nabla u|^{2} .
\end{aligned}
$$

Como

$$
\operatorname{div}\left(p(x)(x-a) \frac{|\nabla u|^{2}}{2}\right)=\nabla p(x) \cdot(x-a) \frac{|\nabla u|^{2}}{2}+p(x) \operatorname{div}\left((x-a) \frac{|\nabla u|^{2}}{2}\right),
$$

segue que

$$
\begin{aligned}
\operatorname{div}(p(x) \nabla u((x-a) \cdot \nabla u)) & =\operatorname{div}(p(x) \nabla u)((x-a) \cdot \nabla u)+\operatorname{div}\left(p(x)(x-a) \frac{|\nabla u|^{2}}{2}\right) \\
& -\nabla p(x) \cdot(x-a) \frac{|\nabla u|^{2}}{2}+p(x)\left(\frac{2-N}{2}\right)|\nabla u|^{2} .
\end{aligned}
$$

Portanto

$$
\begin{aligned}
\operatorname{div}(p(x) \nabla u)((x-a) \cdot \nabla u)= & \operatorname{div}\left(p(x) \nabla u((x-a) \cdot \nabla u)-(x-a) p(x) \frac{|\nabla u|^{2}}{2}\right) \\
& +\nabla p(x) \cdot(x-a) \frac{|\nabla u|^{2}}{2}+p(x)\left(\frac{N-2}{2}\right)|\nabla u|^{2} .
\end{aligned}
$$

De maneira análoga, prova-se que

$$
\begin{aligned}
\operatorname{div}(q(x) \nabla v)((x-a) \cdot \nabla v)= & \operatorname{div}\left(q(x) \nabla v((x-a) \cdot \nabla v)-(x-a) q(x) \frac{|\nabla v|^{2}}{2}\right) \\
& +\nabla q(x) \cdot(x-a) \frac{|\nabla v|^{2}}{2}+q(x)\left(\frac{N-2}{2}\right)|\nabla v|^{2}
\end{aligned}
$$


Por outro lado,

$$
\begin{aligned}
\operatorname{div}((x-a) F(u, v)) & =N F(u, v)+\sum_{i=1}^{N}\left(x_{i}-a_{i}\right) \frac{\partial}{\partial x_{i}} F(u, v) \\
& =N F(u, v)+\sum_{i=1}^{N}\left(x_{i}-a_{i}\right)\left(F_{u}(u, v) \frac{\partial u}{\partial x_{i}}+F_{v}(u, v) \frac{\partial v}{\partial x_{i}}\right) \\
& =N F(u, v)+((x-a) \cdot \nabla u) F_{u}(u, v)+((x-a) \cdot \nabla v) F_{v}(u, v),
\end{aligned}
$$

$\log 0$

$$
((x-a) \cdot \nabla u) F_{u}(u, v)+((x-a) \cdot \nabla v) F_{v}(u, v)=\operatorname{div}((x-a) F(u, v))-N F(u, v) .
$$

Como $(u, v)=(0,0)$ em $\partial \Omega$, temos que $F(u(x), v(x))=0$ sempre que $x \in \partial \Omega$. Além disso, se $y \in \partial \Omega$ e $w \in T_{y}(\partial \Omega)$, considere

$$
\lambda:(-\varepsilon, \varepsilon) \rightarrow \partial \Omega
$$

uma curva diferenciável com $\lambda(0)=y$ e $\lambda^{\prime}(0)=w$. Desse modo, $u(\lambda(t))=0$ sempre que $t \in(-\varepsilon, \varepsilon)$ e consequentemente

$$
\left.\frac{d}{d t} u(\lambda(t))\right|_{t=0}=\nabla u(\lambda(0)) \cdot \lambda^{\prime}(0)=\nabla u(y) \cdot w=0 .
$$

Como $w \in T_{y}(\partial \Omega)$ é qualquer e $T_{y}(\partial \Omega)$ é uma superfície $(n-1)$-dimensional, o gradiente de $u$ restrito à fronteira de $\Omega$ só tem componente normal, isto é

$$
\nabla u=(\nabla u \cdot \eta) \eta
$$

em que $\eta$ é o vetor normal unitário exterior a $\partial \Omega$. Portanto

$$
|\nabla u|^{2}=(\nabla u \cdot \eta) \eta \cdot(\nabla u \cdot \eta) \eta=(\nabla u \cdot \eta)^{2}(\eta \cdot \eta)=(\nabla u \cdot \eta)^{2} .
$$

Também vale que

$$
((x-a) \cdot \nabla u)(\nabla u \cdot \eta)=(\nabla u \cdot \eta)^{2}(\eta \cdot(x-a)) .
$$


Da mesma maneira, prova-se que

$$
|\nabla v|^{2}=(\nabla v \cdot \eta)^{2} \quad \text { e }((x-a) \cdot \nabla v)(\nabla v \cdot \eta)=(\nabla v \cdot \eta)^{2}(\eta \cdot(x-a))
$$

Multiplicando a equação $-\operatorname{div}(p(x) \nabla u)=F_{u}(u, v)$ por $((x-a) \cdot \nabla u)$ e a equação $-\operatorname{div}(q(x) \nabla v)=F_{v}(u, v)$ por $((x-a) \cdot \nabla v)$ e somando as duas, temos que

$$
\begin{aligned}
& -\operatorname{div}(p(x) \nabla u)((x-a) \cdot \nabla u)-\operatorname{div}(q(x) \nabla v)((x-a) \cdot \nabla v) \\
& =((x-a) \cdot \nabla u) F_{u}(u, v)+((x-a) \cdot \nabla v) F_{v}(u, v) .
\end{aligned}
$$

Utilizando as equações (3.3), (3.4), (3.5) e integrando em $\Omega$ a equação acima, temos que

$$
\begin{aligned}
& \int \operatorname{div}\left(p(x) \nabla u((x-a) \cdot \nabla u)-(x-a) p(x) \frac{|\nabla u|^{2}}{2}\right) \\
& +\int \operatorname{div}\left(q(x) \nabla v((x-a) \cdot \nabla v)-(x-a) q(x) \frac{|\nabla v|^{2}}{2}\right) \\
& +\frac{1}{2} \int \nabla p(x) \cdot(x-a)|\nabla u|^{2}+\frac{1}{2} \int \nabla q(x) \cdot(x-a)|\nabla v|^{2} \\
& +\left(\frac{N-2}{2}\right) \int\left(p(x)|\nabla u|^{2}+q(x)|\nabla v|^{2}\right) \\
& =-\int(\operatorname{div}((x-a) F(u, v))-N F(u, v)) .
\end{aligned}
$$

Mas, usando (3.6), (3.7), (3.8) e o Teorema do Divergente, a equação acima pode ser reescrita como

$$
\begin{aligned}
& \frac{(N-2)}{2} \int\left(p(x)|\nabla u|^{2}+q(x)|\nabla v|^{2}\right) \\
& +\frac{1}{2} \int\left(\nabla p(x) \cdot(x-a)|\nabla u|^{2}+\nabla q(x) \cdot(x-a)|\nabla v|^{2}\right) \\
& +\frac{1}{2} \int_{\partial \Omega} p(x)(\nabla u \cdot \eta)^{2}(x-a) \cdot \eta+q(x)(\nabla v \cdot \eta)^{2}(x-a) \cdot \eta d S \\
& =-\int_{\partial \Omega} F(u, v)(x-a) \cdot \eta d S+N \int F(u, v) .
\end{aligned}
$$

Lembrando que $F(u, v)=0$ em $\partial \Omega$ e multiplicando a equação acima por $2 /(N-2)$ obtemos a igualdade desejada.

Estabelecido o lema acima, podemos então provar o resultado de não existência como se segue: 
Demonstração do Teorema 3.1: Suponha, por contradição, que o problema $(P Q)$ admita uma solução $Z=(u, v) \operatorname{com} u, v>0$ em $\Omega$. Uma conta direta mostra que

$$
\int\left(p(x)|\nabla u|^{2}+q(x)|\nabla v|^{2}\right)=\int\langle A Z, Z\rangle_{\mathbb{R}^{2}}+\int|u|^{\alpha}|v|^{\beta} .
$$

Definindo

$$
F(u, v):=\frac{1}{2}\langle A Z, Z\rangle_{\mathbb{R}^{2}}+\frac{1}{2^{*}}|u|^{\alpha}|v|^{\beta}
$$

e substituindo a equação (3.9) na identidade do Lema 3.1 temos que

$$
\begin{aligned}
& \left(1-\frac{2^{*}}{2}\right) \int\langle A Z, Z\rangle_{\mathbb{R}^{2}}+\frac{1}{N-2} \int_{\partial \Omega}\left(p(x)(\nabla u \cdot \eta)^{2}+q(x)(\nabla v \cdot \eta)^{2}\right)(x-a) \cdot \eta d S \\
& +\frac{1}{N-2} \int\left(|\nabla u|^{2} \nabla p(x) \cdot(x-a)+|\nabla v|^{2} \nabla q(x) \cdot(x-a)\right)=0 .
\end{aligned}
$$

Como $\left(1-2^{*} / 2\right)<0, p$ e $q$ são tais que $\nabla p(x) \cdot(x-a) \geq 0$ e $\nabla q(x) \cdot(x-a) \geq 0$ e o domínio $\Omega$ é estrelado com respeito ao ponto $a$, ou seja, $(x-a) \cdot \eta>0$, devemos ter

$$
\int\langle A Z, Z\rangle_{\mathbb{R}^{2}} \geq 0
$$

Observe inicialmente que, se $\mu_{2}<0$, seque de (3.1) que

$$
\langle A Z(x), Z(x)\rangle_{\mathbb{R}^{2}} \leq \mu_{2}|Z(x)|^{2} \leq 0
$$

para todo $x \in \Omega$. Mas isto implica que $Z=(0,0)$, contrariando a positividade da solução. Logo, precisamos somente considerar o caso em que $\mu_{2}=0$. Se em (3.1) tomarmos $Z_{1}=(1,0) \in \mathbb{R}^{2}$, teremos $b=\left\langle A Z_{1}, Z_{1}\right\rangle_{\mathbb{R}^{2}} \leq \mu_{2}\left|Z_{1}\right|^{2}=0$, de modo que $b \leq 0$. O mesmo procedimento com $Z_{2}=(0,1)$ mostra que $d \leq 0$. Além disso, como $\mu_{2}$ é autovalor da matriz $A$, segue que

$$
\operatorname{det}\left(\begin{array}{cc}
b-\mu_{2} & c \\
c & d-\mu_{2}
\end{array}\right)=0
$$

e portanto $c^{2}=b d$.

A prova agora será dividida em 2 casos:

Caso 1: $b=d=0$.

Neste caso, como $c^{2}=b d$, concluímos que $A=0$. Deste modo, a equação que provém do 
Lema 3.1 toma a seguinte forma

$$
\begin{aligned}
& \frac{1}{N-2} \int_{\partial \Omega}\left(p(x)|\nabla u \cdot \eta|^{2}+q(x)|\nabla v \cdot \eta|^{2}\right)(x-a) \cdot \eta d S \\
+ & \frac{1}{N-2} \int\left(|\nabla u|^{2} \nabla p(x) \cdot(x-a)+|\nabla v|^{2} \nabla q(x) \cdot(x-a)\right)=0 .
\end{aligned}
$$

Como por hipótese $\nabla p(x) \cdot(x-a) \geq 0, \nabla q(x) \cdot(x-a) \geq 0$ e $\Omega$ é um domínio estrelado com respeito ao ponto $a$, ou seja, $(x-a) \cdot \eta>0$, concluímos que

$$
|\nabla u \cdot \eta|=|\nabla v \cdot \eta|=0
$$

Integrando a primeira equação do sistema (PS2) e utilizando o Teorema do Divergente obtemos

$$
\frac{1}{2^{*}} \int u|u|^{\alpha-2}|v|^{\beta}=-\int \operatorname{div}(p(x) \nabla u)=\int_{\partial \Omega} p(x) \nabla u \cdot \eta d S=0,
$$

portanto concluímos que

$$
\int u|u|^{\alpha-2}|v|^{\beta}=0
$$

o que contradiz $u, v>0$.

Caso 2: $b<0$ ou $d<0$.

Este caso é um pouco mais delicado. Vamos obter uma contradição supondo que $b<0$, porque o caso $d<0$ é análogo.

Da definição da matriz $A$ e de (3.10), segue que

$$
\int\langle A Z, Z\rangle_{\mathbb{R}^{2}}=b|u|_{2}^{2}+2 c\langle u, v\rangle_{L^{2}(\Omega)}+d|v|_{2}^{2} \geq 0
$$

em que $\langle\cdot, \cdot\rangle_{L^{2}(\Omega)}$ denota o produto interno canônico de $L^{2}(\Omega)$. Uma vez que $2 c\langle u, v\rangle_{L^{2}(\Omega)} \leq$ $2|c||u|_{2}|v|_{2}$, então

$$
b|u|_{2}^{2}+2|c||u|_{2}|v|_{2}+d|v|_{2}^{2} \geq b|u|_{2}^{2}+2 c\langle u, v\rangle_{L^{2}(\Omega)}+d|v|_{2}^{2} \geq 0 .
$$

Como $c^{2}=b d$, então $4 c^{2}|u|_{2}^{2}-4 b d|u|_{2}^{2}=0$. Assim, olhando para o lado esquerdo da expressão acima como uma parábola na variável $|u|_{2}$, e lembrando que $b<0$, concluímos que $b|u|_{2}^{2}+2|c||u|_{2}|v|_{2}+d|v|_{2}^{2} \leq 0$. Logo os termos do meio da inequação são nulos, o que implica

$$
c\langle u, v\rangle_{L^{2}(\Omega)}=|c||u|_{2}^{2}|v|_{2}^{2} .
$$


Mas esta última igualdade só ocorre quando $u$ e $v$ são múltiplos. Como $u>0$, existe $\delta \in \mathbb{R} \backslash\{0\}$ tal que $u=\delta v$. Portanto

$$
|v|_{2}^{2}\left(b \delta^{2}+2 c \delta+d\right)=0
$$

em que esta última igualdade e o fato de que $c^{2}=b d$ implicam em $\delta=-c / b$. Logo, no lado direito da primeira equação em $(P Q)$ devemos ter

$$
b u+c v=b \delta v+c v=b(-c / b) v+c v=0 .
$$

Concluímos, então, que a função $u \in H_{0}^{1}(\Omega)$ satisfaz

$$
-\operatorname{div}(p(x) \nabla u)=\frac{\alpha}{2^{*}|\delta|^{\beta}} u|u|^{2^{*}-2}, \quad x \in \Omega
$$

Mas isto contradiz um resultado provado por Hadiji-Yazidi em [24, Lema 4.1], que afirma que o problema acima não possui solução positiva. Deste modo, a solução $Z=(u, v)$ do início da prova não pode ter as duas componentes positivas

\subsection{Algumas relações importantes}

Nesta seção vamos estabelecer a existência de uma solução para o problema $(P Q)$. Denotaremos por $\left(H_{0}^{1}\right)^{2}$ o espaço $H_{0}^{1}(\Omega) \times H_{0}^{1}(\Omega)$ munido da norma

$$
\|(u, v)\|_{\left(H_{0}^{1}\right)^{2}}^{2}=\|u\|_{H_{0}^{1}(\Omega)}^{2}+\|v\|_{H_{0}^{1}(\Omega)}^{2}
$$

em que $\|u\|_{H_{0}^{1}(\Omega)}^{2}=\int|\nabla u|^{2}$. Lembremos que uma solução fraca de $(P Q)$ é um par $(u, v) \in$ $H_{0}^{1}(\Omega) \times H_{0}^{1}(\Omega)$ satisfazendo

$$
\begin{aligned}
& \int p(x) \nabla u \nabla \varphi+\int q(x) \nabla v \nabla \zeta-\int b u \varphi-\int c v \varphi-\int c u \zeta \\
& -\int d v \zeta-\frac{\alpha}{2^{*}} \int|u|^{\alpha-2}|v|^{\beta} u \varphi-\frac{\beta}{2^{*}} \int|u|^{\alpha}|v|^{\beta-2} v \zeta=0,
\end{aligned}
$$


para todo $(\varphi, \zeta) \in\left(H_{0}^{1}\right)^{2}$. Soluções fracas do problema $(P Q)$ são pontos críticos do funcional

$$
I(u, v):=\frac{1}{2} \int\left(p(x)|\nabla u|^{2}+q(x)|\nabla v|^{2}\right)-\frac{1}{2} \int\langle A U, U\rangle_{\mathbb{R}^{2}}-\frac{1}{2^{*}} \int\left(u^{+}\right)^{\alpha}\left(v^{+}\right)^{\beta} .
$$

Vamos definir

$$
S_{p, q}:=\inf _{\substack{(u, v) \in\left(H_{0}^{1}\right)^{2} \\(u, v) \neq(0,0)}} \frac{\int\left(p(x)|\nabla u|^{2}+q(x)|\nabla v|^{2}\right)}{\left(\int\left(u^{+}\right)^{\alpha}\left(v^{+}\right)^{\beta}\right)^{2 / 2^{*}}} .
$$

Aqui, e no que se segue, vamos assumir que o quociente acima é infinito sempre que o denominador for igual a zero. Definimos ainda

$$
S_{A}:=\inf _{\substack{Z=(u, v) \in\left(H_{0}^{1}\right)^{2} \\(u, v) \neq(0,0)}} J(u, v)
$$

em que

$$
J(u, v):=\frac{\int\left(p(x)|\nabla u|^{2}+q(x)|\nabla v|^{2}\right)-\int\langle A Z, Z\rangle_{\mathbb{R}^{2}}}{\left(\int\left(u^{+}\right)^{\alpha}\left(v^{+}\right)^{\beta}\right)^{2 / 2^{*}}} .
$$

Do mesmo modo que no Capítulo 1, vamos denotar por $S$ o seguinte número

$$
S=\inf \left\{\int|\nabla u|^{2}: u \in H_{0}^{1}(\Omega), \int|u|^{2^{*}}=1\right\} .
$$

Começamos observando que, sob as hipóteses do Teorema 3.2, existe uma constante $c>0$ tal que

$$
\left(\int\left(u^{+}\right)^{\alpha}\left(v^{+}\right)^{\beta}\right)^{1 / 2^{*}} \leq c\|(u, v)\|_{\left(H_{0}^{1}\right)^{2}} .
$$

De fato, para todo $(s, t) \in \mathbb{R}^{2}$, temos que $|s|^{\alpha}|t|^{\beta} \leq|s|^{\alpha+\beta}+|t|^{\alpha+\beta}$. Assim

$$
\begin{aligned}
\left(\int\left(u^{+}\right)^{\alpha}\left(v^{+}\right)^{\beta}\right)^{1 / 2^{*}} & \leq\left(\int\left(|u|^{2^{*}}+|v|^{2^{*}}\right)\right)^{1 / 2^{*}} \\
& \leq \frac{1}{S^{1 / 2}}\left[\left(\int|\nabla u|^{2}\right)^{\frac{2^{*}}{2}}+\left(\int|\nabla v|^{2}\right)^{\frac{2^{*}}{2}}\right]^{1 / 2^{*}} \\
& \leq \frac{c_{1}}{S^{1 / 2}}\left(\int|\nabla u|^{2}+\int|\nabla v|^{2}\right)^{1 / 2} \\
& =c_{2}\|(u, v)\|_{\left(H_{0}^{1}\right)^{2}} .
\end{aligned}
$$


Além disso, ao supormos $\mu_{2}<\min \left\{\lambda_{1, p}, \lambda_{1, q}\right\}$, podemos nos valer de (3.1), da desigualdade de Poincaré e de (3.15) para obter, para todo $Z=(u, v) \neq(0,0)$,

$$
\begin{aligned}
J(u, v) & =\frac{\int\left(p(x)|\nabla u|^{2}+q(x)|\nabla v|^{2}\right)-\int\langle A Z, Z\rangle_{\mathbb{R}^{2}}}{\left(\int\left(u^{+}\right)^{\alpha}\left(v^{+}\right)^{\beta}\right)^{2 / 2^{*}}} \\
& \geq \frac{\int p(x)|\nabla u|^{2}+\int q(x)|\nabla v|^{2}-\mu_{2} \int\left(u^{2}+v^{2}\right)}{\left(\int|u|^{\alpha}|v|^{\beta}\right)^{2 / 2^{*}}} \\
& \geq \frac{p_{0}\left(1-\frac{\mu_{2}}{\lambda_{1, p}}\right) \int|\nabla u|^{2}+q_{0}\left(1-\frac{\mu_{2}}{\lambda_{1, q}}\right) \int|\nabla v|^{2}}{c \int\left(|\nabla u|^{2}+|\nabla v|^{2}\right)} \\
& \geq \frac{c_{3}}{c}>0, \quad
\end{aligned}
$$

em que $c_{3}:=\min \left\{p_{0}\left(1-\mu_{2} / \lambda_{1, p}\right), q_{0}\left(1-\mu_{2} / \lambda_{1, q}\right)\right\}$. Tomando o ínfimo em $Z$ e lembrando a definição de $S_{A}$ dada em (3.14), concluímos que

$$
S_{A} \geq c_{4}>0
$$

Na demonstração do Teorema 3.2 vamos utilizar alguns lemas. No primeiro deles vamos utilizar a seguinte relação entre $S$ e $S_{p, q}$ :

Lema 3.2. Vale a seguinte estimativa

$$
S p_{0}^{\frac{\alpha}{2^{*}}} q_{0}^{\frac{\beta}{2^{*}}}\left[\left(\frac{\alpha}{\beta}\right)^{\frac{\beta}{2^{*}}}+\left(\frac{\alpha}{\beta}\right)^{\frac{-\alpha}{2^{*}}}\right] \leq S_{p, q}
$$

Demonstração: Observemos inicialmente que, se $s, t>0$, então

$$
\begin{aligned}
p_{0}\left(\frac{s}{t}\right)^{\frac{2 \beta}{2^{*}}}+q_{0}\left(\frac{s}{t}\right)^{\frac{-2 \alpha}{2^{*}}} & =p_{0} \frac{s^{\frac{2 \beta}{2^{*}}}}{t^{\frac{2 \beta}{2^{*}}}}+q_{0} \frac{s^{\frac{-2 \alpha}{2^{*}}}}{t^{\frac{-2 \alpha}{2^{*}}}} \\
& =\frac{p_{0} s^{\frac{2 \beta}{2^{*}}} s^{\frac{2 \alpha}{2^{*}}}}{\left(s^{\alpha} t^{\beta}\right)^{\frac{2}{2^{*}}}}+\frac{q_{0} s^{\frac{-2 \alpha}{2^{*}}} t^{\frac{2 \beta}{2^{*}}}}{t^{\frac{-2 \alpha}{2^{*}}} t^{\frac{2 \beta}{2^{*}}}} \\
& =\frac{p_{0} s^{2}+q_{0} t^{2}}{\left(s^{\alpha} t^{\beta}\right)^{\frac{2}{2^{*}}}}
\end{aligned}
$$


ou seja,

$$
\frac{p_{0} s^{2}+q_{0} t^{2}}{\left(s^{\alpha} t^{\beta}\right)^{\frac{2}{2^{*}}}}=p_{0}\left(\frac{s}{t}\right)^{\frac{2 \beta}{2^{*}}}+q_{0}\left(\frac{s}{t}\right)^{\frac{-2 \alpha}{2^{*}}} .
$$

Consideremos agora a função $g$ definida por

$$
g(r)=p_{0} r^{\frac{2 \beta}{2^{*}}}+q_{0} r^{\frac{-2 \alpha}{2^{*}}}, \quad r>0
$$

Temos que

$$
g^{\prime}(r)=\frac{2 \beta}{2^{*}} p_{0} r^{\frac{2 \beta}{2^{*}}-1}-\frac{2 \alpha}{2^{*}} q_{0} r^{\frac{-2 \alpha}{2^{*}}-1}=\frac{2 \beta}{2^{*}} p_{0} r^{\frac{\beta-\alpha}{2^{*}}}-\frac{2 \alpha}{2^{*}} q_{0} r^{\frac{-3 \alpha-\beta}{2^{*}}} .
$$

Assim, $g^{\prime}\left(r_{0}\right)=0$ desde que

$$
r_{0}=\sqrt{\frac{\alpha q_{0}}{\beta p_{0}}}
$$

Como $\lim _{r \rightarrow 0^{+}} g(r)=\lim _{r \rightarrow+\infty} g(r)=+\infty$ e $r_{0}$ é o único ponto crítico de $g$, temos que $r_{0}$ é ponto de mínimo da função $g$.

Seja agora $\left(u_{n}, v_{n}\right) \subset\left(H_{0}^{1}\right)^{2}$ uma sequência tal que

$$
S_{p, q}=\lim _{n \rightarrow \infty} \frac{\int\left(p(x)\left|\nabla u_{n}\right|^{2}+q(x)\left|\nabla v_{n}\right|^{2}\right)}{\left(\int\left(u_{n}^{+}\right)^{\alpha}\left(v_{n}^{+}\right)^{\beta}\right)^{\frac{2}{2^{*}}}}
$$

e seja $s_{n}>0$ satisfazendo

$$
\int\left|u_{n}\right|^{2^{*}}=s_{n}^{2^{*}} \int\left|v_{n}\right|^{2^{*}}
$$

Se definirmos $w_{n}=s_{n} v_{n}$, a equação acima pode ser reescrita como

$$
\int\left|u_{n}\right|^{2^{*}}=\int\left|w_{n}\right|^{2^{*}}
$$

Podemos agora utilizar a desigualdade de Young como abaixo

$$
\left|u_{n}\right|^{\alpha}\left|w_{n}\right|^{\beta} \leq \frac{\left(\left|u_{n}\right|^{\alpha}\right)^{\frac{2^{*}}{\alpha}}}{\frac{2^{*}}{\alpha}}+\frac{\left(\left|w_{n}\right|^{\beta}\right)^{\frac{2^{*}}{\beta}}}{\frac{2^{*}}{\beta}}=\frac{\alpha}{2^{*}}\left|u_{n}\right|^{2^{*}}+\frac{\beta}{2^{*}}\left|w_{n}\right|^{2^{*}}
$$


e a igualdade em (3.17) para obter

$$
\begin{aligned}
\left(\int\left(u_{n}^{+}\right)^{\alpha}\left(w_{n}^{+}\right)^{\beta}\right)^{\frac{2}{2^{*}}} & \leq\left(\frac{\alpha}{2^{*}} \int\left|u_{n}\right|^{2^{*}}+\frac{\beta}{2^{*}} \int\left|w_{n}\right|^{2^{*}}\right)^{\frac{2}{2^{*}}} \\
& =\left(\int\left|u_{n}\right|^{2^{*}}\right)^{\frac{2}{2^{*}}} \\
& =\left(\int\left|w_{n}\right|^{2^{*}}\right)^{\frac{2}{2^{*}}} .
\end{aligned}
$$

Portanto, usando a desigualdade acima, as definições de $w_{n}, g, p(x), q(x)$ e $S$ obtemos

$$
\begin{aligned}
& \frac{\int\left(p(x)\left|\nabla u_{n}\right|^{2}+q(x)\left|\nabla v_{n}\right|^{2}\right)}{\left(\int\left(u_{n}^{+}\right)^{\alpha}\left(v_{n}^{+}\right)^{\beta}\right)^{\frac{2}{2^{*}}}} \geq s_{n}^{\frac{2 \beta}{2^{*}}} \frac{\int\left(p(x)\left|\nabla u_{n}\right|^{2}+q(x)\left|\nabla v_{n}\right|^{2}\right)}{\left(\int\left(u_{n}^{+}\right)^{\alpha}\left(w_{n}^{+}\right)^{\beta}\right)^{\frac{2}{2^{*}}}} \\
& \geq s_{n}^{\frac{2 \beta}{2^{*}}} p_{0} \frac{\int\left|\nabla u_{n}\right|^{2}}{\left(\int\left|u_{n}\right|^{2^{*}}\right)^{\frac{2}{2^{*}}}}+s_{n^{\frac{-2 \alpha}{2^{*}}}} q_{0} \frac{\int\left|\nabla w_{n}\right|^{2}}{\left(\int\left|w_{n}\right|^{2^{*}}\right)^{\frac{2}{2^{*}}}} \\
& \geq g\left(s_{n}\right) S .
\end{aligned}
$$

Lembrando que $r_{0}=\sqrt{\frac{\alpha q_{0}}{\beta p_{0}}}$ é o mínimo de $g$, segue que

$$
\begin{aligned}
\frac{\int\left(p(x)\left|\nabla u_{n}\right|^{2}+q(x)\left|\nabla v_{n}\right|^{2}\right)}{\left(\int\left(u_{n}^{+}\right)^{\alpha}\left(v_{n}^{+}\right)^{\beta}\right)^{\frac{2}{2^{*}}}} & \geq g\left(\sqrt{\frac{\alpha q_{0}}{\beta p_{0}}}\right) S \\
& =S\left[p_{0}\left(\sqrt{\frac{\alpha q_{0}}{\beta p_{0}}}\right)^{\frac{2 \beta}{2^{*}}}+q_{0}\left(\sqrt{\frac{\alpha q_{0}}{\beta p_{0}}}\right)^{-\frac{2 \alpha}{2^{*}}}\right] \\
& =S p_{0}^{\frac{\alpha}{2^{*}}} q_{0}^{\frac{\beta}{2^{*}}}\left[\left(\frac{\alpha}{\beta}\right)^{\frac{\beta}{2^{*}}}+\left(\frac{\alpha}{\beta}\right)^{\frac{-\alpha}{2^{*}}}\right] .
\end{aligned}
$$

Passando ao limite, obtemos

$$
S p_{0}^{\frac{\alpha}{2^{*}}} q_{0}^{\frac{\beta}{2^{*}}}\left[\left(\frac{\alpha}{\beta}\right)^{\frac{\beta}{2^{*}}}+\left(\frac{\alpha}{\beta}\right)^{\frac{-\alpha}{2^{*}}}\right] \leq S_{p, q}
$$

o que conclui a prova do lema. 


\subsection{Existência de solução}

Provaremos nosso resultado de existência nesta seção. Vamos procurar pontos críticos não triviais para o funcional $I \in C^{1}\left(\left(H_{0}^{1}\right)^{2}, \mathbb{R}\right)$ definido por

$$
I(u, v):=\frac{1}{2} \int\left(p(x)|\nabla u|^{2}+q(x)|\nabla v|^{2}\right)-\frac{1}{2} \int\langle A Z, Z\rangle_{\mathbb{R}^{2}}-\frac{1}{2^{*}} \int\left(u^{+}\right)^{\alpha}\left(v^{+}\right)^{\beta},
$$

onde $Z=(u, v)$. O primeiro passo é verificar que $I$ satisfaz uma das condições geométricas do Teorema do Passo da Montanha:

Lema 3.3. Se o maior autovalor $\mu_{2}$ de A satisfaz $\mu_{2}<\min \left\{\lambda_{1, p}, \lambda_{1, q}\right\}$, então existem $\rho$, $r>0$ tais que

$$
I(u, v) \geq \rho>0 \quad \text { sempre que }\|(u, v)\|_{\left(H_{0}^{1}\right)^{2}}=r .
$$

Demonstração: Devido à continuidade da imersão $H_{0}^{1}(\Omega) \hookrightarrow L^{2^{*}}(\Omega)$, existe uma constante $c>0$ tal que $|u|_{2^{*}}^{2^{*}} \leq c^{2^{*}}\|u\|^{2^{*}}$. Usando a desigualdade $\left(s^{+}\right)^{\alpha}\left(t^{+}\right)^{\beta} \leq|s|^{\alpha+\beta}+|t|^{\alpha+\beta}$, para $(s, t) \in \mathbb{R}^{2}$, e a desigualdade (3.1) obtemos

$$
\begin{aligned}
I(u, v) & =\frac{1}{2} \int\left(p(x)|\nabla u|^{2}+q(x)|\nabla v|^{2}\right)-\frac{1}{2} \int\langle A Z, Z\rangle_{\mathbb{R}^{2}}-\frac{1}{2^{*}} \int\left(u^{+}\right)^{\alpha}\left(v^{+}\right)^{\beta} \\
& \geq \frac{1}{2} \int\left(p(x)|\nabla u|^{2}+q(x)|\nabla v|^{2}\right)-\frac{\mu_{2}}{2} \int\left(u^{2}+v^{2}\right)-\frac{1}{2^{*}} \int\left(|u|^{2^{*}}+|v|^{2^{*}}\right) \\
& \geq \Lambda_{p}\|u\|^{2}+\Lambda_{q}\|v\|^{2}-\frac{1}{2^{*}} c^{2^{*}}\|u\|^{2^{*}}-\frac{1}{2^{*}} c^{2^{*}}\|v\|^{2^{*}} \\
& =\left(\Lambda_{p}-\frac{c^{2^{*}}}{2^{*}}\|u\|^{2^{*}-2}\right)\|u\|^{2}+\left(\Lambda_{q}-\frac{c^{2^{*}}}{2^{*}}\|v\|^{2^{*}-2}\right)\|v\|^{2},
\end{aligned}
$$

em que

$$
\Lambda_{p}:=\frac{p_{0}}{2}\left(1-\frac{\mu_{2}}{\lambda_{1, p}}\right)>0 \quad \text { e } \quad \Lambda_{q}:=\frac{q_{0}}{2}\left(1-\frac{\mu_{2}}{\lambda_{1, q}}\right)>0 .
$$

Escolhemos $\|u\|=r_{1}>0$ e $\|v\|=r_{2}>0$ tais que $\left(\Lambda_{p}-c^{2^{*}} / 2^{*}\|u\|^{2^{*}-2}\right)>0$ e $\left(\Lambda_{q}-\right.$ $\left.c^{2^{*}} / 2^{*}\|v\|^{2^{*}-2}\right)>0$. O resultado segue tomando $r=\sqrt{r_{1}^{2}+r_{2}^{2}}>0$.

Na sequência, vamos considerar, para cada $u \in H_{0}^{1}(\Omega) \backslash\{0\}$, os quocientes

$$
Q_{\lambda, p}(u):=\frac{\|u\|_{p}^{2}-\lambda|u|_{2}^{2}}{|u|_{2^{*}}^{2}} \quad \text { e } \quad Q_{\lambda, q}(u):=\frac{\|u\|_{q}^{2}-\lambda|u|_{2}^{2}}{|u|_{2^{*}}^{2}}
$$


bem como a seguinte função

$$
w_{\varepsilon}(x)=\frac{\psi(x) \varepsilon^{\frac{N-2}{4}}}{\left[\varepsilon+|x-a|^{2}\right]^{\frac{N-2}{2}}},
$$

em que $\varepsilon>0, \psi \in C_{0}^{\infty}(\Omega), 0 \leq \psi \leq 1, \psi \equiv 1$ em $B(a, l), \psi \equiv 0$ em $\Omega \backslash \overline{B(a, 2 l)}$ e $l>0$ é tal que $\overline{B(a, 2 l)} \subset \Omega$.

Enunciamos abaixo a relação entre as duas funções acima. A prova do lema foi apresentada no capítulo anterior.

Lema 3.4. Valem as seguintes estimativas quando $\varepsilon \rightarrow 0^{+}$

$$
Q_{\lambda, p}\left(w_{\varepsilon}\right) \leq \begin{cases}p_{0} S-\lambda \frac{K_{3}}{K_{2}} \varepsilon+o(\varepsilon), & N \geq 5, k>2, \\ p_{0} S-\left(\lambda-\frac{A_{2}}{K_{3}} \beta_{2}\right) \frac{K_{3}}{K_{2}} \varepsilon+o(\varepsilon), & N \geq 5 \text { e } k=2, \\ p_{0} S-\lambda \frac{\omega_{4}}{2 K_{2}} \varepsilon|\log \varepsilon|+o(\varepsilon|\log \varepsilon|), & N=4 \text { e } k>2, \\ p_{0} S-\left(\lambda-4 \beta_{2}\right) \frac{\omega_{4}}{2 K_{2}} \varepsilon|\log \varepsilon|+o(\varepsilon|\log \varepsilon|), & N=4, k=2,\end{cases}
$$

em que $\omega_{4}$ é a área da esfera unitária de $\mathbb{R}^{4}$ e $K_{2}, K_{3}$ a $A_{2}$ dependem somente de $N$. Além disso, valem estimativas análogas para $Q_{\lambda, q}$, com $\gamma_{2}$ no lugar de $\beta_{2}$.

Utilizando as estimativas acima podemos provar o resultado seguinte:

Lema 3.5. Seja $\vartheta(N, k, j)$ conforme definido em (3.2). Se o menor autovalor $\mu_{1} d a$ matriz $A$ é tal que $\mu_{1}>\vartheta(N, k, j)$, então

$$
S_{A}<S_{p, q}
$$

Além disso, existe $\left(u_{1}, v_{1}\right) \in\left(H_{0}^{1}\right)^{2}$ tal que

$$
\max _{t \geq 0} I\left(t u_{1}, t v_{1}\right)<\frac{1}{N}\left(\frac{S_{p, q}}{2}\right)^{N / 2} .
$$

Demonstração: Dados $B, C>0$ definimos $Z_{\varepsilon}=\left(B w_{\varepsilon}, C w_{\varepsilon}\right)$. Utilizando o fato de que 
$w_{\varepsilon}^{+}=w_{\varepsilon}$ e a relação $(3.1)$, obtemos

$$
\begin{aligned}
J\left(B w_{\varepsilon}, C w_{\varepsilon}\right) & =\frac{B^{2} \int p(x)\left|\nabla w_{\varepsilon}\right|^{2}+C^{2} \int q(x)\left|\nabla w_{\varepsilon}\right|^{2}-\int\left\langle A Z_{\varepsilon}, Z_{\varepsilon}\right\rangle_{\mathbb{R}^{2}}}{\left(B^{\alpha} C^{\beta}\right)^{\frac{2}{2^{*}}}\left(\int\left|w_{\varepsilon}\right|^{2^{*}}\right)^{2 / 2^{*}}} \\
& \leq \frac{B^{2}\left(\int p(x)\left|\nabla w_{\varepsilon}\right|^{2}-\int \mu_{1}\left|w_{\varepsilon}\right|^{2}\right)+C^{2}\left(\int q(x)\left|\nabla w_{\varepsilon}\right|^{2}-\int \mu_{1}\left|w_{\varepsilon}\right|^{2}\right)}{\left(B^{\alpha} C^{\beta}\right)^{\frac{2}{2^{*}}}\left(\int\left|w_{\varepsilon}\right|^{2^{*}}\right)^{2 / 2^{*}}} \\
& =\frac{B^{2} Q_{\mu_{1}, p}\left(w_{\varepsilon}\right)+C^{2} Q_{\mu_{1}, q}\left(w_{\varepsilon}\right)}{\left(B^{\alpha} C^{\beta}\right)^{\frac{2}{2^{*}}}} .
\end{aligned}
$$

Observando agora que $\mu_{1}>\vartheta(N, k, j)$, podemos usar o lema anterior e os mesmos argumentos do Capítulo 1 concluir que

$$
Q_{\mu_{1}, p}\left(w_{\varepsilon}\right)<p_{0} S, \quad Q_{\mu_{1}, q}\left(w_{\varepsilon}\right)<q_{0} S
$$

Segue das estimativas acima e de (3.16) que

$$
J\left(B w_{\varepsilon}, C w_{\varepsilon}\right)<\frac{\left(B^{2} p_{0}+C^{2} q_{0}\right) S}{\left(B^{\alpha} C^{\beta}\right)^{\frac{2}{2^{*}}}}=\left[p_{0}\left(\frac{B}{C}\right)^{\frac{2 \beta}{2^{*}}}+q_{0}\left(\frac{B}{C}\right)^{-\frac{2 \alpha}{2^{*}}}\right] S .
$$

Assim, escolhendo $B, C>0$ tais que

$$
\left(\frac{B}{C}\right)^{2}=\frac{q_{0} \alpha}{p_{0} \beta}
$$

podemos usar a definição de $S_{A}$ e o Lema 3.2 para obter

$$
S_{A}<\left[p_{0}\left(\frac{q_{0} \alpha}{p_{0} \beta}\right)^{\frac{\beta}{2^{*}}}+q_{0}\left(\frac{q_{0} \alpha}{p_{0} \beta}\right)^{-\frac{\alpha}{2^{*}}}\right] S=S p_{0}^{\frac{\alpha}{2^{*}}} q_{0}^{\frac{\beta}{2^{*}}}\left[\left(\frac{\alpha}{\beta}\right)^{\frac{\beta}{2^{*}}}+\left(\frac{\alpha}{\beta}\right)^{\frac{-\alpha}{2^{*}}}\right] \leq S_{p, q}
$$

o que encerra a primeira parte.

Agora, para $\varepsilon>0$, seja $\widetilde{w}_{\varepsilon}:=\frac{w_{\varepsilon}}{\left|w_{\varepsilon}\right|_{2^{*}}}$. Dessa forma

$$
\begin{aligned}
I\left(t B \widetilde{w}_{\varepsilon}, t C \widetilde{w}_{\varepsilon}\right) & \leq \frac{t^{2} B^{2}}{2} \int p(x)\left|\nabla \widetilde{w}_{\varepsilon}\right|^{2}+\frac{t^{2} C^{2}}{2} \int q(x)\left|\nabla \widetilde{w}_{\varepsilon}\right|^{2} \\
& -\frac{t^{2}}{2}\left(B^{2}+C^{2}\right) \mu_{1} \int\left|\widetilde{w}_{\varepsilon}\right|^{2}-\frac{1}{2^{*}} t^{2^{*}} B^{\alpha} C^{\beta} \int\left|\widetilde{w}_{\varepsilon}\right|^{2^{*}}
\end{aligned}
$$


Observemos agora que, para $Q_{p}, Q_{q}>0$, a função $g$ definida por

$$
g(t)=\frac{t^{2}}{2}\left(B^{2} Q_{p}+C^{2} Q_{q}\right)-\frac{1}{2^{*}} t^{2^{*}} B^{\alpha} C^{\beta}, \quad t>0,
$$

é tal que $g^{\prime}(t)=t\left(B^{2} Q_{p}+C^{2} Q_{q}\right)-t^{2^{*}-1} B^{\alpha} C^{\beta}$, e portanto seu único ponto crítico é

$$
t_{0}:=\left(\frac{B^{2} Q_{p}+C^{2} Q_{q}}{B^{\alpha} C^{\beta}}\right)^{\frac{1}{2^{*}-2}} .
$$

Como $g^{\prime \prime}\left(t_{0}\right)=\left(2-2^{*}\right)\left(B^{2} Q_{p}+C^{2} Q_{q}\right)<0$, concluímos que $t_{0}$ é ponto de máximo. Assim o valor máximo da função $g$ é

$$
\begin{aligned}
g\left(t_{0}\right) & =\frac{1}{2}\left(\frac{B^{2} Q_{p}+C^{2} Q_{q}}{2 B^{\alpha} C^{\beta}}\right)^{\frac{2}{2^{*}-2}}\left(B^{2} Q_{p}+C^{2} Q_{p}\right)-\frac{1}{2^{*}}\left(\frac{B^{2} Q_{p}+C^{2} Q_{q}}{2 B^{\alpha} C^{\beta}}\right)^{\frac{2^{*}}{2^{*}-2}} B^{\alpha} C^{\beta} \\
& =\frac{1}{N}\left(\frac{B^{2} Q_{p}+C^{2} Q_{q}}{2\left(B^{\alpha} C^{\beta}\right)^{\frac{2}{2^{*}}}}\right)^{\frac{N}{2}} .
\end{aligned}
$$

Portanto, escolhendo $Q_{p}=Q_{\mu_{1}, p}\left(\widetilde{w}_{\varepsilon}\right)$ e $Q_{q}=Q_{\mu_{1}, q}\left(\widetilde{w}_{\varepsilon}\right)$, segue da expressão acima, (3.19), (3.16), a escolha de $B$ e $C$ e do Lema 3.2 que

$$
\begin{aligned}
\sup _{t \geq 0} I\left(t B \widetilde{w}_{\varepsilon}, t C \widetilde{w}_{\varepsilon}\right) & \leq \frac{1}{N}\left(\frac{B^{2} Q_{p}+C^{2} Q_{q}}{2\left(B^{\alpha} C^{\beta}\right)^{\frac{1}{2^{*}}}}\right)^{\frac{N}{2}}<\frac{1}{N}\left(\frac{\left(p_{0} B^{2}+q_{0} C^{2}\right) S}{2\left(B^{\alpha} C^{\beta}\right)^{\frac{2}{2^{*}}}}\right)^{\frac{N}{2}} \\
& =\frac{1}{N}\left[\frac{S}{2}\left(p_{0}\left(\frac{B}{C}\right)^{\frac{2 \beta}{2^{*}}}+q_{0}\left(\frac{B}{C}\right)^{\frac{-2 \alpha}{2^{*}}}\right)\right]^{\frac{N}{2}} \\
& =\frac{1}{N}\left[\frac{S}{2} p_{0}^{\frac{\alpha}{2^{*}}} q_{0}^{\frac{\beta}{2^{*}}}\left(\left(\frac{\alpha}{\beta}\right)^{\frac{\beta}{2^{*}}}+\left(\frac{\alpha}{\beta}\right)^{\frac{-\alpha}{2^{*}}}\right)\right]^{\frac{N}{2}} \leq \frac{1}{N}\left(\frac{S_{p, q}}{2}\right)^{\frac{N}{2}} .
\end{aligned}
$$

Assim, fazendo $\left(u_{1}, v_{1}\right)=\left(B \widetilde{w}_{\varepsilon}, C \widetilde{w}_{\varepsilon}\right)$, com $\varepsilon>0$ suficientemente pequeno, temos que

$$
\max _{t \geq 0} J\left(t u_{1}, t v_{1}\right)<\frac{1}{N}\left(\frac{S_{p, q}}{2}\right)^{N / 2} .
$$

Estamos prontos para provar o nosso resultado de existência de solução. 
Prova do Teorema 3.2: Seja $\left(u_{1}, v_{1}\right) \in\left(H_{0}^{1}\right)^{2}$ dado pelo lema anterior. Temos que

$$
I\left(t u_{1}, t v_{1}\right)=\frac{t^{2}}{2} \int\left(p(x)\left|\nabla u_{1}\right|^{2}+q(x)\left|\nabla v_{1}\right|^{2}\right)-\frac{t^{2}}{2} \int\left\langle A U_{1}, U_{1}\right\rangle_{\mathbb{R}^{2}}-\frac{t^{2^{*}}}{2^{*}} \int\left(u_{1}^{+}\right)^{\alpha}\left(v_{1}^{+}\right)^{\beta} .
$$

Como $\lim _{t \rightarrow \infty} I\left(t u_{1}, t v_{1}\right)=-\infty$, então existe um par $(\widetilde{u}, \widetilde{v}) \in\left(H_{0}^{1}\right)^{2}$ tal que $\|(\widetilde{u}, \widetilde{v})\|_{\left(H_{0}^{1}\right)^{2}}>r$, para $r$ dado no Lema 3.18, e $I(\widetilde{u}, \widetilde{v})<0$. Vamos definir

$$
c_{0}:=\inf _{g \in \Gamma} \max _{0 \leq t \leq 1} I(g(t))
$$

em que

$$
\Gamma=\left\{g \in C\left([0,1],\left(H_{0}^{1}\right)^{2}\right): g(0)=0, g(1)=(\widetilde{u}, \widetilde{v})\right\}
$$

Segue do Teorema do Passo da Montanha que existe uma sequência $\left(Z_{n}\right)=\left(u_{n}, v_{n}\right) \subset$ $\left(H_{0}^{1}\right)^{2}$ tal que

$$
I\left(u_{n}, v_{n}\right) \rightarrow c_{0}>0 \quad \text { e } \quad I^{\prime}\left(u_{n}, v_{n}\right) \rightarrow 0
$$

Afirmamos que $\left(u_{n}, v_{n}\right)$ é limitada em $\left(H_{0}^{1}\right)^{2}$. De fato, para $n$ suficientemente grande, $\left\|I^{\prime}\left(u_{n}, v_{n}\right)\right\|_{\left(\left(H_{0}^{1}\right)^{2}\right)^{-1}} \leq 2^{*}$. Assim

$$
\left|I^{\prime}\left(u_{n}, v_{n}\right)\left(u_{n}, v_{n}\right)\right| \leq\left\|I^{\prime}\left(u_{n}, v_{n}\right)\right\|_{\left(\left(H_{0}^{1}\right)^{2}\right)^{-1}}\left\|\left(u_{n}, v_{n}\right)\right\|_{\left(H_{0}^{1}\right)^{2}} \leq 2^{*}\left\|\left(u_{n}, v_{n}\right)\right\|_{\left(H_{0}^{1}\right)^{2}}
$$

e portanto

$$
\left\|\left(u_{n}, v_{n}\right)\right\|_{\left(H_{0}^{1}\right)^{2}} \geq-\frac{1}{2^{*}} I^{\prime}\left(u_{n}, v_{n}\right)\left(u_{n}, v_{n}\right) .
$$

Como $I\left(u_{n}, v_{n}\right)=c_{0}+o(1)$, temos que

$$
\begin{aligned}
o(1)+c_{0}+\left\|\left(u_{n}, v_{n}\right)\right\|_{\left(H_{0}^{1}\right)^{2}} & \geq I\left(u_{n}, v_{n}\right)-\frac{1}{2^{*}} J^{\prime}\left(u_{n}, v_{n}\right)\left(u_{n}, v_{n}\right) \\
& =\frac{1}{2} \int\left(p(x)\left|\nabla u_{n}\right|^{2}+q(x)\left|\nabla v_{n}\right|^{2}\right) \\
& -\frac{1}{2} \int\left\langle A Z_{n}, Z_{n}\right\rangle_{\mathbb{R}^{2}}-\frac{1}{2^{*}} \int\left(u_{n}^{+}\right)^{\alpha}\left(v_{n}^{+}\right)^{\beta} \\
& -\frac{1}{2^{*}} \int\left(p(x)\left|\nabla u_{n}\right|^{2}+q(x)\left|\nabla v_{n}\right|^{2}\right) \\
& +\frac{1}{2^{*}} \int\left\langle A Z_{n}, Z_{n}\right\rangle_{\mathbb{R}^{2}}+\frac{1}{2^{*}} \int\left(u_{n}^{+}\right)^{\alpha}\left(v_{n}^{+}\right)^{\beta} .
\end{aligned}
$$


Usando (3.1) e o fato de que $\mu_{2}<\min \left\{\lambda_{1, p}, \lambda_{1, q}\right\}$ a desigualdade acima fica

$$
\begin{aligned}
o(1)+c_{0}+\left\|\left(u_{n}, v_{n}\right)\right\|_{\left(H_{0}^{1}\right)^{2}} & \geq \frac{1}{2} \int\left(p(x)\left|\nabla u_{n}\right|^{2}+q(x)\left|\nabla v_{n}\right|^{2}\right) \\
& +\mu_{2}\left(\frac{1}{2^{*}}-\frac{1}{2}\right) \int\left(u_{n}^{2}+v_{n}^{2}\right) \\
& \geq\left(1-\frac{\mu_{2}}{\lambda_{1, p}}\right)\left(\frac{1}{2}-\frac{1}{2^{*}}\right) p_{0}\left\|u_{n}\right\|^{2} \\
& +\left(1-\frac{\mu_{2}}{\lambda_{1, q}}\right)\left(\frac{1}{2}-\frac{1}{2^{*}}\right) q_{0}\left\|v_{n}\right\|^{2},
\end{aligned}
$$

e assim concluímos que a sequência $\left(u_{n}, v_{n}\right)$ é limitada em $\left(H_{0}^{1}\right)^{2}$.

A menos de subsequência, temos que

$$
\begin{aligned}
& u_{n} \rightarrow u_{0} \quad \text { e } \quad v_{n} \rightarrow v_{0} \quad \text { em } \quad H_{0}^{1}(\Omega), \\
& u_{n} \rightarrow u_{0} \quad \text { e } \quad v_{n} \rightarrow v_{0} \quad \text { em } \quad L^{\sigma}(\Omega), \quad 2 \leq \sigma<2^{*}, \\
& u_{n}(x) \rightarrow u_{0}(x) \quad \text { e } \quad v_{n}(x) \rightarrow v_{0}(x) \text { q.t.p. em } \Omega \text {, } \\
& \left|u_{n}(x)\right| \leq h_{s}(x) \quad \text { e } \quad\left|v_{n}(x)\right| \leq h_{r}(x), \quad x \in \Omega,
\end{aligned}
$$

em que $h_{s} \in L^{2^{*}-1}(\Omega), h_{r} \in L^{2^{*}-1}(\Omega)$. Se tomarmos $h(x):=\max _{x \in \Omega}\left\{h_{s}(x), h_{r}(x)\right\}$, então $h \in L^{2^{*}-1}(\Omega)$. Assim, para qualquer $\varphi \in C_{0}^{\infty}(\Omega)$,

$$
\begin{aligned}
u_{n}^{\alpha-1}(x) v_{n}^{\beta}(x) \varphi(x) & \longrightarrow u_{0}^{\alpha-1}(x) v_{0}^{\beta}(x) \varphi(x) \quad \text { q.t.p. em } \Omega, \\
\left|u_{n}\right|^{\alpha-1}\left|v_{n}\right|^{\beta}|\varphi| \leq|\varphi|_{\infty} h^{2^{*}-1} \in L^{1}(\Omega) . &
\end{aligned}
$$

Pelo Teorema da Convergência Dominada, $\int\left|u_{n}\right|^{\alpha-2} u_{n}\left|v_{n}\right|^{\beta} \varphi \rightarrow \int\left|u_{0}\right|^{\alpha-2} u_{0}\left|v_{0}\right|^{\beta} \varphi$ e, por densidade

$$
\lim _{n \rightarrow+\infty} \int\left|u_{n}\right|^{\alpha-2} u_{n}\left|v_{n}\right|^{\beta} \varphi=\int\left|u_{0}\right|^{\alpha-2} u_{0}\left|v_{0}\right|^{\beta} \varphi, \quad \forall \varphi \in H_{0}^{1}(\Omega) .
$$

De maneira análoga, provamos que

$$
\lim _{n \rightarrow+\infty} \int\left|u_{n}\right|^{\alpha}\left|v_{n}\right|^{\beta-2} v_{n} \zeta=\int\left|u_{0}\right|^{\alpha}\left|v_{0}\right|^{\beta-2} v_{0} \zeta, \quad \forall \zeta \in H_{0}^{1}(\Omega) .
$$

Além disso, as convergências fracas em $H_{0}^{1}(\Omega)$ e fortes em $L^{2}(\Omega)$ implicam que

$$
\left\langle u_{n}, \varphi\right\rangle_{p}+\left\langle v_{n}, \zeta\right\rangle_{q}-\int\left\langle A Z_{n}, W\right\rangle_{\mathbb{R}^{2}}=\left\langle u_{0}, \varphi\right\rangle_{p}+\left\langle v_{0}, \zeta\right\rangle_{q}-\int\left\langle A Z_{0}, W\right\rangle_{\mathbb{R}^{2}}+o(1),
$$


em que $Z_{0}=\left(u_{0}, v_{0}\right)$ e $W=(\varphi, \zeta)$. Como $I^{\prime}\left(u_{n}, v_{n}\right) \rightarrow 0$, obtemos

$$
\begin{aligned}
o(1) & =I^{\prime}\left(u_{n}, v_{n}\right)(\varphi, \zeta) \\
& =\left\langle u_{n}, \varphi\right\rangle_{p}+\left\langle v_{n}, \zeta\right\rangle_{q}-\int\left\langle A Z_{n}, W\right\rangle_{\mathbb{R}^{N}}-\frac{\alpha}{2^{*}} \int\left|u_{n}\right|^{\alpha-2} u_{n}\left|v_{n}\right|^{\beta} \varphi \\
& -\frac{\beta}{2^{*}} \int\left|u_{n}\right|^{\alpha}\left|v_{n}\right|^{\beta-2} v_{n} \zeta \\
& =\left\langle u_{0}, w_{1}\right\rangle_{p}+\left\langle v_{0}, w_{2}\right\rangle_{q}-\int\left\langle A Z_{0}, W\right\rangle_{\mathbb{R}^{N}}-\frac{\alpha}{2^{*}} \int\left|u_{0}\right|^{\alpha-2} u_{0}\left|v_{0}\right|^{\beta} \varphi \\
& -\frac{\beta}{2^{*}} \int\left|u_{0}\right|^{\alpha}\left|v_{0}\right|^{\beta-2} v_{0} \zeta+o(1),
\end{aligned}
$$

para qualquer par $(\varphi, \zeta) \in\left(H_{0}^{1}\right)^{2}$, o que prova que $\left(u_{0}, v_{0}\right)$ é solução fraca de $(P Q)$.

Afirmação: não pode ocorrer $\left(u_{0}, v_{0}\right)=(0,0)$.

De fato, seja

$$
l=\lim _{n \rightarrow \infty} \int\left(p(x)\left|\nabla u_{n}\right|^{2}+q(x)\left|\nabla v_{n}\right|^{2}\right) \geq 0 .
$$

Se $\left(u_{0}, v_{0}\right)=(0,0)$ então $u_{n}, v_{n} \rightarrow 0$ fortemente em $L^{2}(\Omega)$. Isso e $J^{\prime}\left(u_{n}, v_{n}\right)\left(u_{n}, v_{n}\right)=o(1)$ implicaria que

$$
\lim _{n \rightarrow+\infty} \int\left(u_{n}^{+}\right)^{\alpha}\left(v_{n}^{+}\right)^{\beta}=\frac{l}{2} .
$$

Assim, subtraindo as duas igualdades abaixo

$$
\begin{gathered}
2 I\left(u_{n}, v_{n}\right)=\int p(x)\left|\nabla u_{n}\right|^{2}+q(x)\left|\nabla v_{n}\right|^{2}-\int\left\langle A Z_{n}, Z_{n}\right\rangle_{\mathbb{R}^{2}}-\frac{2}{2^{*}} \int\left(u_{n}^{+}\right)^{\alpha}\left(v_{n}^{+}\right)^{\beta}, \\
o(1)=\int p(x)\left|\nabla u_{n}\right|^{2}+q(x)\left|\nabla v_{n}\right|^{2}-\int\left\langle A Z_{n}, Z_{n}\right\rangle_{\mathbb{R}^{2}}-\int\left(u_{n}^{+}\right)^{\alpha}\left(v_{n}^{+}\right)^{\beta}
\end{gathered}
$$

e passando ao limite, obteríamos

$$
2 c_{0}=\left(1-\frac{2}{2^{*}}\right) \frac{l}{2} .
$$

Lembrando que

$$
\int p(x)\left|\nabla u_{n}\right|^{2}+q(x)\left|\nabla v_{n}\right|^{2} \geq S_{p, q}\left(\int\left(u_{n}^{+}\right)^{\alpha}\left(v_{n}^{+}\right)^{\beta}\right)^{\frac{2}{2^{*}}}
$$


e passando ao limite novamente vem

$$
\frac{c_{0}}{\left(1-\frac{1}{2^{*}}\right)} \geq S_{p, q}\left[\frac{c_{0}}{\left(1-\frac{2}{2^{*}}\right)}\right]^{\frac{2}{2^{*}}}
$$

donde

$$
c_{0} \geq \frac{1}{N}\left(\frac{S_{p, q}}{2}\right)^{\frac{N}{2}},
$$

o que contradiz o Lema 3.5. Esta contradição prova a afirmação.

Suponha agora que $u_{0} \equiv 0$. A primeira equação em $(P Q)$ nos fornece $c v_{0}=0$. Segue então da afirmação acima que $c=0$, e portanto $b, d \in\left\{\mu_{1}, \mu_{2}\right\}$. Nessas condições, $v_{0}$ satisfaz

$$
\left\{\begin{aligned}
-\operatorname{div}(q(x) \nabla v) & =d v, \quad \Omega, \\
v & =0, \quad \partial \Omega .
\end{aligned}\right.
$$

Como estamos supondo $0<\mu_{1} \leq \mu_{2}<\lambda_{1, q}$, teríamos $v_{0}=0$, o que contradiz a afirmação acima. De maneira análoga mostra-se que não pode ocorrer $v_{0} \equiv 0$, consequentemente as duas componentes $u_{0}$ e $v_{0}$ são não triviais.

Vamos mostrar que as componentes da solução são não negativas. Como o par $\left(u_{0}, v_{0}\right) \in\left(H_{0}^{1}\right)^{2}$ é ponto crítico de $I$, fazendo $Z_{0}^{-}=\left(u_{0}^{-}, v_{0}^{-}\right)$, em que $w^{-}(x):=\min \{w(x), 0\}$, temos que

$$
\begin{aligned}
0 & =I^{\prime}\left(u_{0}, v_{0}\right)\left(u_{0}^{-}, v_{0}^{-}\right) \\
& =\int\left(p(x) \nabla u_{0} \nabla u_{0}^{-}+q(x) \nabla v_{0} \nabla u_{0}^{-}\right)-\int\left\langle A Z_{0}, Z_{0}^{-}\right\rangle_{\mathbb{R}^{2}} \\
& \geq\left(1-\frac{\mu_{2}}{\lambda_{1, p}}\right)\left(\frac{1}{2}-\frac{1}{2^{*}}\right) p_{0}\left\|u_{0}^{-}\right\|^{2}+\left(1-\frac{\mu_{2}}{\lambda_{1, q}}\right)\left(\frac{1}{2}-\frac{1}{2^{*}}\right) q_{0}\left\|v_{0}^{-}\right\|^{2} \geq 0,
\end{aligned}
$$

o que nos fornece $\left\|u_{0}^{-}\right\|=\left\|v_{0}^{-}\right\|=0$, ou ainda, $u_{0} \geq 0$ e $v_{0} \geq 0$.

Resta mostrar que as componentes são positivas. Se $b \leq 0$, então a primeira equação de $(P Q)$ se escreve como

$$
\operatorname{div}\left(p(x) \nabla u_{0}\right)+b u_{0}=-c v_{0}-\frac{\alpha}{2^{*}} u_{0}\left|u_{0}\right|^{\alpha-2}\left|v_{0}\right|^{\beta} \leq 0
$$

em que usamos $c \geq 0$. Como $u_{0} \geq 0$, segue do Princípio do Máximo Forte que, se existisse um ponto $x \in \Omega$ tal que $u_{0}(x)=0$, então $u_{0} \equiv 0$ em $\Omega$, o que não ocorre. Logo $u_{0}$ é 
positiva. Se $b \geq 0$ temos

$$
\operatorname{div}\left(p(x) \nabla u_{0}\right)=-b u_{0}-c v_{0}-\frac{\alpha}{2^{*}} u_{0}\left|u_{0}\right|^{\alpha-2}\left|v_{0}\right|^{\beta} \leq 0
$$

e a positividade de $u_{0}$ segue novamente do Princípio do Máximo Forte. A prova de que $v_{0}>0$ é análoga. 


\section{Bibliografia}

[1] Alves, C. O., de Morais Filho, D. C. and Souto, M. A. S., On systems of elliptic equations involving subcritical Sobolev exponents, Nonlinear Analysis 42 (2000), 771787.

[2] Ambrosetti, A., Brézis, H. and Cerami, G., Combined effects of concave and convex nonlinearities in some elliptic problems, J. Funct. Anal. 122(1994), 519 - 543.

[3] Aubin, T., Problème isopérimétrique et espaces de Sobolev, J. Differential Geometry., 11, (1976) $573-598$.

[4] Bahri, A. and Coron, J. M., On a nonlinear elliptic equation involving the critical Sobolev exponent: The effect of the topology of the domain, Comm. Pure Appl. Math. 41 (1988), 253-294.

[5] Boccardo, L., Figueiredo, D.G., Some remarks on a system of quasilinear elliptic equation, NoDEA Nonlinear Differ. Equ. Appl. 9, 309-323 (2002).

[6] Bouchekif, M., Hamzaoui, Y., On Elliptic System Involving Critical Sobolev Exponent and Weight, Mediterranean Journal of Mathematics, 2013, 497-517.

[7] Brézis, H., Elliptic equations with limiting Sobolev exponents, The impact of topology, Comm. Pure Appl. Math., 39, 1986, S17-S39.

[8] Brézis, H., Lieb, E., A relation between pointwise convergence of functions and convergence of functionals, Proc. Amer. Math. Soc. 88 (1983), 486-490. 
[9] Brézis, H., and Niremberg. L., Positive solutions of nonlinear elliptic equations involving critical Sobolev exponents, Comm. Pure Appl. Math. 36(1993) 437-477.

[10] Capozzi, A., Fortunato, L., Positive solutions of nonlinear elliptic equations involving critical Sobolev exponents, Comm. Pure Appl. Math. 36(1993) 437-477.

[11] Cerami, G., Solimini, S. and Struwe, M., Some existence results for superlinear elliptic boundary value problems involving critical exponents, J. Func. Anal. 69 (1986), 289306.

[12] Coron, J. M., Topologie et cas limite des injections de Sobolev, C. R. Acad. Sc. Paris., 299, 1985, 209-211.

[13] Chabrowski, J., Yang, J., On the Neumann problem for an elliptic system of equations involving the critical Sobolev exponent, Colloq. Math. , 90 (2001), 19-35.

[14] Figueiredo, D.G. Gossez, J.P. and Ubilla, P., Local superlinearity for indefinite semilinear elliptic problems, J. Funct. Anal. 199 (2003), 452 - 467.

[15] Figueiredo, D.G. Gossez, J.P. and Ubilla, P., Local "superlinearity" and "sublinearity" for the p-Laplacian J. Funct. Anal. 257 (2009), 721 - 752.

[16] Figueiredo, D.G. Gossez, J.P. and Ubilla, P., Multiplicity results for a family of semilinear elliptic problems under local superlinearity and sublinearity, J. Eur. Math Soc. 8, (2006) $269-286$.

[17] Figueiredo, D.G., Semilinear elliptic systems. Nonlinear Funct. Anal. Appl., held at ICTP of Trieste, (April 21-May 9, 1997).

[18] Furtado, M.F., Miyagaki, O.H., Silva, J.P., On a class of nonlinear elliptic equations with fast increasing weight and critical growth, J. Differential Equations 249 (2010), 1035-1055.

[19] Furtado, M.F., Ruviaro, R. and Silva, J.P., Two solutions for an elliptic equation with fast increasing weight and concave-convex nonlinearities, J. M. A. A. - 416, (2014), 698-709.

[20] Garcia Azorero, J. and Peral Alonso, I., Existence and non-uniqueness for the pLaplacian: Nonlinear eigenvalues, Comm. Partial Diff. Eq. 12 (1987), 1389-1430. 
[21] Garcia Azorero, J. and Peral Alonso, I., Multiplicity of solutions for elliptic problem with critical exponent or with a nonsymmetric term, Trans. Amer. Math. Soc. 323 (1991), 877-895.

[22] Gonçalves, J.V. Miyagaki, O.H., Multiple positive solutions for semilinear elliptic equations in $\mathbb{R}^{n}$ involving subcritical exponents, Nonlinear Analysis, Theory, Methods \& Applications, Vol. 32, No. 1, pp. 41-51, 1998.

[23] Gueda, M. and Veron, L., Quasilinear elliptic equations involving critical Sobolev exponents, Nonlinear Anal. 13 (1989), 879-902.

[24] Hadiji, R., Yazidi, H., Problem with Critical Sobolev Exponent and with Weight, Chinese Annals of Mathematics, 28 (2007), 327-352.

[25] Han, P., High-energy positive solutions for a critical growth Dirichlet problem in noncontractible domains Nonlinear Analysis 60 (2005) 369-387.

[26] Han, P., The effect of the domain topology on the number of positive solutions of some elliptic systems involving critical Sobolev exponents, preprint.

[27] Lazzo, M., Solutions positives multiples pour une équation elliptique non linéaire avec léxposant critique de Sobolev, C. R. Acad. Sci. Paris 314 (1992), 61-64.

[28] Pohozaev, R. S. J., Eingenfunctions of the equation $\Delta u+\lambda f(u)=0$, Soviet Math. Doklady, 6, 1965, 1408-1411.

[29] Rabinowitz, A., Minimax Methods in Critical Point Theory with Applications to Differential Equations. American Mathematical Society, 1988.

[30] Rey, O., A multiplicity result for a variational problem with lack of compactness, Nonlinear Anal. 13 (1989), 1241-1249.

[31] Struwe, M., A global compactness result for elliptic boundary value problems involving limiting nonlinearities. Math. Z. 187, (1984) 511-517.

[32] Talenti, G., Best constants in Sobolev inequality, Ann. Mat. Pura Appl. 110, (1976), $353-372$.

[33] Tehrani, H., On elliptic equations with nonlinearities that are sum of a sublinear and superlinear term, volume dedicated to Dr. S. Shahshahani, Iran (2002) 123-131. 
[34] Tarantello, G., Nodal solutions of semilinear elliptic equations with critical exponent, Diff. Int. Equations 5 (1992), 25-42.

[35] Willem, M., Minimax Theorems. Birkhäuser, 1996. 